D. Haran, M. Jarden and F. Pop

Nagoya Math. J.

Vol. 194 (2009), 91-147

\title{
THE ABSOLUTE GALOIS GROUP OF THE FIELD OF TOTALLY $S$-ADIC NUMBERS
}

\author{
DAN HARAN, MOSHE JARDEN AND FLORIAN POP
}

\begin{abstract}
For a finite set $S$ of primes of a number field $K$ and for $\sigma_{1}, \ldots, \sigma_{e} \in$ $\operatorname{Gal}(K)$ we denote the field of totally $S$-adic numbers by $K_{\mathrm{tot}, S}$ and the fixed field of $\sigma_{1}, \ldots, \sigma_{e}$ in $K_{\text {tot }, S}$ by $K_{\text {tot }, S}(\boldsymbol{\sigma})$. We prove that for almost all $\boldsymbol{\sigma} \in$ $\operatorname{Gal}(K)^{e}$ the absolute Galois group of $K_{\text {tot }, S}(\boldsymbol{\sigma})$ is the free product of $\hat{F}_{e}$ and a free product of local factors over $S$.
\end{abstract}

\section{Introduction}

The Inverse Galois Problem asks whether every finite group is realizable over $\mathbb{Q}$. Although this has been shown to be true for many finite groups, including the symmetric and alternating groups (Hilbert), we are still very far from the solution of the problem. One could ask, more generally, what is the structure of the absolute Galois group of $\mathbb{Q}$. Here we do not even have a plausible conjecture.

However, we do know the structure of the absolute Galois group of certain distinguished algebraic extensions of $\mathbb{Q}$, or, more generally, of a countable Hilbertian field $K$. We fix a separable closure $K_{s}$ and an algebraic closure $\tilde{K}$ of $K$ and let $\operatorname{Gal}(K)=\operatorname{Gal}\left(K_{s} / K\right)$ be the absolute Galois group of $K$. Our goal is to explore the absolute Galois groups of large algebraic extensions of $K$ having interesting diophantine or arithmetical properties.

Our study is motivated by two earlier results. By the free generators theorem $\operatorname{Gal}\left(K_{s}(\boldsymbol{\sigma})\right)$ is, for almost all $\boldsymbol{\sigma} \in \operatorname{Gal}(K)^{e}$, the free profinite group $\hat{F}_{e}$ on $e$ generators (Jarden [FrJ, Thm. 18.5.6]). On the other hand, if $K$ is a global field and $S_{1}$ is a finite set of primes of $K$, then the absolute Galois group $\operatorname{Gal}\left(K_{\mathrm{tot}, S_{1}}\right)$ of the maximal $S_{1}$-adic extension of $K$ is a free product

Received November 14, 2006.

Revised October 25, 2007, May 6, 2008.

Accepted August 21, 2008.

2000 Mathematics Subject Classification: 12E30.

Research supported by the Minkowski Center for Geometry at Tel Aviv University, established by the Minerva Foundation, by an ISF grant, by the European Community Marie Curie Research Training Network GTEM, and by NSF research grant DMS 0401056. 
of local groups (Pop [Pop4, Thm. 3]). In this work we simultaneously generalize both results and prove that $\operatorname{Gal}\left(K_{s}(\boldsymbol{\sigma}) \cap K_{\text {tot, } S_{1}}\right)$ is, for almost all $\boldsymbol{\sigma} \in \operatorname{Gal}(K)^{e}$, the free product of $\hat{F}_{e}$ and a free product of local groups.

Here is a detailed account of our result.

\section{The main theorem}

For each $e$-tuple $\boldsymbol{\sigma}=\left(\sigma_{1}, \ldots, \sigma_{e}\right) \in \operatorname{Gal}(K)^{e}$ we denote the fixed field in $K_{s}$ (resp. $\left.\tilde{K}\right)$ of $\sigma_{1}, \ldots, \sigma_{e}$ by $K_{s}(\boldsymbol{\sigma})$ (or $\tilde{K}(\boldsymbol{\sigma})$ if $\operatorname{char}(K)=0$ ). We know that for almost all $\boldsymbol{\sigma} \in \mathrm{Gal}(K)^{e}$ the field $K_{s}(\boldsymbol{\sigma})$ is PAC [FrJ, Thm. 18.6.1] and $\operatorname{Gal}\left(K_{s}(\boldsymbol{\sigma})\right) \cong \hat{F}_{e}[\mathrm{FrJ}, \mathrm{Thm}$. 18.5.6]. Here "almost all" is meant in the sense of the Haar measure of $\operatorname{Gal}(K)^{e}$ and we say that a field $M$ is PAC if every absolutely irreducible variety $V$ defined over $M$ has an $M$-rational point. The PAC property of the field $K_{s}(\boldsymbol{\sigma})$ implies that if $w$ is a nontrivial valuation of $K_{s}(\boldsymbol{\sigma})$, then the Henselian closure of $K_{s}(\boldsymbol{\sigma})$ at $w$ is $K_{s}$ [FrJ, Cor. 11.5.5].

To bring valuations into the game we consider a finite set $S_{1}$ of absolute values of $K$. For each $v \in S_{1}$ let $\hat{K}_{v}$ be a completion of $K$ at $v$. Then $\hat{K}_{v}$ is $\mathbb{C}, \mathbb{R}$, a finite extension of $\mathbb{Q}_{p}$, or a finite extension of $\mathbb{F}_{p}((t))$ for some prime number $p$. The latter case does not occur if $\operatorname{char}(K)=0$. The case where the completion is $\mathbb{C}$ is uninteresting in each of the following results, so we assume $\hat{K}_{v} \neq \mathbb{C}$ for each $v \in S_{1}$. Assume all of the $\hat{K}_{v}$ 's are contained in a common field. For each $v \in S_{1}$ set $K_{v}=\tilde{K} \cap \hat{K}_{v}$. Then $K_{v}$ is a real closure of $K$ at $v$ or a Henselian closure of $K$ at $v$.

First consider the field

$$
M^{\prime}=K_{s}(\boldsymbol{\sigma}) \cap \bigcap_{v \in S_{1}} K_{v}^{\rho_{v}}
$$

where $(\boldsymbol{\sigma}, \boldsymbol{\rho}) \in \operatorname{Gal}(K)^{e+\left|S_{1}\right|}$ are taken at random. Then

$$
\operatorname{Gal}\left(M^{\prime}\right) \cong \hat{F}_{e} * \prod_{v \in S_{1}} \operatorname{Gal}\left(K_{v}^{\rho_{v}}\right)
$$

where $*$ and $*$ denote free products in the sense explained after the Main Theorem. (See [Gey, Thm. 4.1] for $e=0$ and [Jar2, Thm. 21.3] in the general case.)

Next we assume that $\operatorname{char}(K)=0$ and that the absolute values in $S_{1}$ are independent and consider the maximal totally $S_{1}$-adic extension of $K$ :

$$
K_{\text {tot }, S_{1}}=\bigcap_{v \in S_{1}} \bigcap_{\rho \in \operatorname{Gal}(K)} K_{v}^{\rho} .
$$


Finally we "dig deeper" to reach the field $K_{\text {tot }, S_{1}}(\boldsymbol{\sigma})=\tilde{K}(\boldsymbol{\sigma}) \cap K_{\text {tot }, S_{1}}$. Then for almost all $\boldsymbol{\sigma}$ the field $K_{\text {tot }, S_{1}}(\boldsymbol{\sigma})$ is pseudo- $S_{1}$-closed (Proposition 12.3). This means that every absolutely irreducible variety $V$ defined over $K_{\text {tot, } S_{1}}(\boldsymbol{\sigma})$ with a simple $K_{v}^{\rho}$-rational point for all $v$ and $\rho$ has a $K_{\text {tot, } S_{1}}(\boldsymbol{\sigma})$-rational point. It follows that $\operatorname{Gal}\left(K_{\text {tot }, S_{1}}(\boldsymbol{\sigma})\right)$ is relatively projective with respect to the set $\left\{\operatorname{Gal}\left(K_{v}^{\rho}\right) \mid v \in S_{1}, \rho \in \operatorname{Gal}(K)\right\}[\mathrm{HJPb}$, Proposition 4.1]. Thus, each finite embedding problem for $\operatorname{Gal}\left(K_{\text {tot, } S_{1}}(\boldsymbol{\sigma})\right)$ which has a local weak solution for each subgroup $\operatorname{Gal}\left(K_{v}^{\rho}\right)$ has a global weak solution. This is a basic ingredient in the proof of the main result of this work:

MAIN THEOREM. Let $K$ be a countable Hilbertian field of characteristic $0, e \geq 0$ an integer, and $S_{1}$ a finite set of independent absolute values of $K$. Then for almost all $\boldsymbol{\sigma} \in \operatorname{Gal}(K)^{e}$ and for each $v \in S_{1}$ there exists a closed subset $R_{v}$ of $\operatorname{Gal}(K)$ such that

$$
\operatorname{Gal}\left(K_{t o t, S_{1}}(\boldsymbol{\sigma})\right)=\operatorname{Gal}(\tilde{K}(\boldsymbol{\sigma})) * \prod_{v \in S_{1}} \prod_{\rho \in R_{v}} \operatorname{Gal}\left(K_{v}^{\rho}\right),
$$

and $R_{v}$ is a system of representatives of $\operatorname{Gal}\left(K_{\text {tot }, S_{1}}(\boldsymbol{\sigma})\right) \backslash \mathrm{Gal}(K)$, and $\left\{K_{v}^{\rho} \mid\right.$ $\left.\rho \in R_{v}\right\}$ is a closed system of representatives of the $\operatorname{Gal}\left(K_{\mathrm{tot}, S_{1}}(\boldsymbol{\sigma})\right)$-orbits of $\left\{K_{v}^{\rho} \mid \rho \in \operatorname{Gal}(K)\right\}$.

Here we use the notation $H \backslash G$ with $G$ a profinite group and $H$ a closed subgroup for the space $\{H g \mid g \in G\}$ of all right cosets of $G$ modulo $H$. The inner free product in (1) is meant here in the sense of Haran-Melnikov ([Har], [Mel]): First, the intersection of distinct factors is trivial, secondly, each continuous function

$$
\varphi_{0}: \bigcup_{v \in S_{1}} \bigcup_{\rho \in R_{v}} \operatorname{Gal}\left(K_{v}^{\rho}\right) \longrightarrow B
$$

into a profinite group $B$ whose restriction to each of the groups $\operatorname{Gal}\left(K_{v}^{\rho}\right)$ is a homomorphism uniquely extends to a continuous homomorphism $\varphi$ : $*_{v \in S_{1}}$ $\circledast_{\rho \in R_{v}} \operatorname{Gal}\left(K_{v}^{\rho}\right) \rightarrow B$.

\section{Sketch of proof}

The proof of the main theorem applies a blend of local and global properties of the field $M=K_{\text {tot }, S_{1}}(\boldsymbol{\sigma})$ and its absolute Galois group. In addition to the local fields $K_{v}, v \in S_{1}$, we consider also $K_{0}=\tilde{K}(\boldsymbol{\sigma})$ as a local field 
of $M$ and write $S_{0}=\{0\}$ and $S=S_{0} \cup S_{1}$. The common feature of the groups $\operatorname{Gal}\left(K_{v}\right), v \in S_{1}$, and $\operatorname{Gal}\left(K_{0}\right)$ is that they are all finitely generated. Beyond that they are of a different nature. Here are the main properties of the fields $K_{v}, v \in S_{1}$, that enter in the proof of the main theorem:

(2a) The groups $\operatorname{Gal}\left(K_{v}\right), v \in S_{1}$, can be recognized group theoretically (up to conjugation and inclusion) by "big quotients" in each free product of the form $\hat{F} * \mathbb{*}_{i=1}^{n} G_{i}$, where $\hat{F}$ is a free finitely generated profinite group and each $G_{i}$ is isomorphic to $\operatorname{Gal}\left(K_{v}\right)$ for some $v \in S_{1}$ (Data 7.1).

(2b) If $K_{v}$ is algebraically closed in a field $F$ and $\operatorname{Gal}(F) \cong \operatorname{Gal}\left(K_{v}\right)$, then $K_{v}$ is an elementary subfield of $F$ (a combination of results of EfratKoenigsmann-Pop and Ax-Kochen-Ershov-Prestel-Roquette).

(2c) For each $v \in S_{1}$ the space of $\operatorname{Gal}(M)$-orbits of $\mathcal{G}_{v}=\left\{\operatorname{Gal}\left(K_{v}^{\rho}\right) \mid \rho \in\right.$ $\operatorname{Gal}(K)\}$ is isomorphic to the Cantor middle third set, in particular it has no isolated points.

The main properties of $K_{0}$ used in the proof are the following:

(3a) $\mathrm{Gal}\left(K_{0}\right)$ is a finitely generated free profinite group.

(3b) $K_{0}$ is pseudo algebraically closed over each set $H \cap A$ where $H$ is a Hilbert subset of $K^{r}$ and $A$ is a nonempty $S_{1}$-adically open subset of $K^{r}$ (Definition 9.3).

In addition to the relative projectivity of the group $\operatorname{Gal}(M)$ drawn from the $\mathrm{P} S_{1} \mathrm{C}$ property of $M$, we also apply the following consequence of being $\mathrm{P} S_{1} \mathrm{C}$ :

(4) Each finite split embedding problem over $M$ can be regularly solved over $M(t)$, where $t$ is transcendental over $M$.

We keep track of the local groups of $\operatorname{Gal}(M)$ by considering the "group pile" $\mathbf{G}=(\operatorname{Gal}(M), \mathcal{G} a l(M, v))_{v \in S}$, where $\mathcal{G} a l(M, 0)$ is the $\operatorname{Gal}(M)$-orbit of $\operatorname{Gal}\left(K_{0}\right)$ and $\mathcal{G} a l(M, v)$ is the $\operatorname{Gal}(K)$-orbit of $\operatorname{Gal}\left(K_{v}\right)$. Finite group piles, $\mathbf{A}=\left(A, \mathcal{A}_{v}\right)_{v \in S}$, are modeled after finite quotients of $\mathbf{G}$ (Section 3). The key step in the proof of the main theorem is proving that each "self-generated" "rigid" finite embedding problem

$$
(\varphi: \mathbf{G} \rightarrow \mathbf{A}, \alpha: \mathbf{B} \rightarrow \mathbf{A})
$$


of group piles which splits group theoretically is solvable. Without loss $A=\operatorname{Gal}(N / M)$ is a Galois group over $M$. Using (4) and (2b) we find a finite Galois extension $P$ of $M(t)$ which solves the group theoretic problem attached to (5) and such that the local structure of $\mathrm{Gal}(P / M(t))$ associated with $S_{1}$ is isomorphic to that of $\mathbf{B}$. Using a theorem of Efrat, we are able to choose $B_{0} \in \mathcal{B}_{0}$ such that $\alpha\left(B_{0}\right)=\varphi\left(\operatorname{Gal}\left(K_{0}\right)\right)$ and $B$ is generated by $B_{0}$ and all of the groups belonging to $\mathcal{B}_{1}$. Let $P_{0}$ be the fixed field in $P$ of the subgroup of $\operatorname{Gal}(P / M(t))$ corresponding to $B_{0}$. Then, we may assume that $B=\operatorname{Gal}(P / M(t))$. An application of Hilbertianity, (2a), (3b), and the rigidity assumption (Section 4) gives a homomorphism $\gamma: \operatorname{Gal}(M) \rightarrow$ $\operatorname{Gal}(P / M(t))$ which commutes with restrictions such that $\gamma\left(\operatorname{Gal}\left(K_{0}\right)\right)=$ $\operatorname{Gal}\left(P / P_{0}\right)$ and $\gamma(\mathcal{G a l}(M, v))=\mathcal{B}_{v}$ for each $v \in S_{1}$. By assumption, the local groups generate $\operatorname{Gal}(P / M(t))$, so $\gamma$ is surjective (Proposition 11.1).

We note that the use of property (3) and the Hilbertianity of $K$ follows [FHV] which proves the main theorem in the special case where $e=0$ and all of the $K_{v}$ with $v \in S_{1}$ are real closed fields.

Now, for each $v \in S_{1}$ we choose a homeomorphic image $T_{v}$ of the Cantor middle third set and construct a free product $G_{T}=\hat{F}_{e} * \prod_{v \in S_{1}} \prod_{t \in T_{v}} G_{T, t}$ with $G_{T, t} \cong \operatorname{Gal}\left(K_{v}\right)$ (Proposition 11.1). We prove that each finite selfgenerated embedding problem for group piles associated with $G_{T}$ is solvable (Proposition 5.3(h)). The same holds for $\operatorname{Gal}(M)$ (Proposition 11.1). It follows by an Iwasawa like argument (Proposition 6.3), that $\operatorname{Gal}(M) \cong$ $\operatorname{Gal}\left(G_{T}\right)$. Consequently, $\operatorname{Gal}(M)$ is a free product of its local groups.

\section{Positive characteristic}

The proof of the main theorem we have just described does not work in positive characteristic $p$. Here the completions of the local fields $K_{v}$ are finite extensions of $\mathbb{F}_{p}((t))$, so the groups $\operatorname{Gal}\left(K_{v}\right)$ are not finitely generated. Another problem is that no analog for the Ax-Kochen-Ershov theorem is known in characteristic $p$.

The special case of the main theorem where $e=0$ was proved by the third author in all characteristics in a unified way [Pop4]. The unified proof is indirect. In order to prove that $\mathrm{Gal}\left(K_{\mathrm{tot}, S_{1}}\right)$ is a product of local groups one chooses a Galois extension $N$ of $K$ in $K_{\text {tot, } S_{1}}$ which is $\mathrm{P} S_{1} \mathrm{C}$ and properly contained in $K_{\text {tot, } S_{1}}$. For example* one may choose $N$ to be the maximal Galois extension $K_{\mathrm{tot}, S_{1}}[\boldsymbol{\sigma}]$ of $K$ in $K_{\mathrm{tot}, S_{1}}(\boldsymbol{\sigma})$, where $\boldsymbol{\sigma}$ is an element of $\operatorname{Gal}(K)^{e}$ chosen at random. Let $N^{\prime}$ be a finite proper extension of $N$ in

\footnotetext{
${ }^{*}$ Our argument at this point differs somewhat from the one given in [Pop4].
} 
$K_{\text {tot, } S_{1}}$. Then $N^{\prime}$ is Hilbertian (by a theorem of Weissauer) and $\mathrm{P} S_{1} \mathrm{C}$ [GeJ, Thm. A]. Under these assumptions [Pop4] proves that $\operatorname{Gal}\left(N^{\prime}\right)$ is isomorphic to the free product of $\hat{F}_{\omega}$ and a free product of local groups. Moreover, it proves that the closed normal subgroup generated by the second factor is the group $\operatorname{Gal}\left(K_{\mathrm{tot}, S_{1}}\right)$ and is isomorphic to the first factor.

\section{The fields $K_{\text {tot }, S_{1}}[\boldsymbol{\sigma}]$}

Another question left open in this work is the structure of the Galois group of the field $K_{\text {tot, } S_{1}}[\boldsymbol{\sigma}]$, where $\boldsymbol{\sigma}$ is taken at random in $\operatorname{Gal}(K)^{e}$. We wish to prove that the structure of that group is given by an analog of (1) in which $\operatorname{Gal}(\tilde{K}(\boldsymbol{\sigma}))$ is replaced by $\operatorname{Gal}\left(K_{s}[\boldsymbol{\sigma}]\right)$. See Remark 12.5 for more details.

\section{$\S 1$. Automorphisms of finitely generated profinite groups}

Let $\Gamma$ be a finitely generated profinite group and $A$ a finite quotient of $\Gamma$. We construct a big quotient $B$ of $\Gamma$ in a uniform way such that each automorphism of $A$ which lifts to an automorphism of $B$ lifts to an automorphism of $\Gamma$.

To that end we consider a positive integer $n$ and observe that $\Gamma$ has only finitely many distinct open subgroups of index $\leq n$ [FrJ, Lemma 16.10.2]. Their intersection $\Gamma_{(n)}$ is an open characteristic subgroup of $\Gamma$ and $\Gamma^{(n)}=$ $\Gamma / \Gamma_{(n)}$ is a finite group. Furthermore, $\Gamma=\Gamma_{(1)} \geq \Gamma_{(2)} \geq \Gamma_{(3)} \geq \cdots$ and $\bigcap_{n=1}^{\infty} \Gamma_{(n)}=1$.

LEMMA 1.1. Let $\Gamma$ be a finitely generated profinite group and $n$ a positive integer. Consider an open normal subgroup $N$ of $\Gamma$ with $N \leq \Gamma_{(n)}$. Then:

(a) $(\Gamma / N)_{(n)}=\Gamma_{(n)} / N$.

(b) Let $N^{\prime}$ be another open normal subgroup of $\Gamma$ such that $\Gamma / N \cong \Gamma / N^{\prime}$. Then $N^{\prime} \leq \Gamma_{(n)}$.

(c) Assume that $\Gamma$ is a closed subgroup of a profinite group $G$ and let $K$ be an open normal subgroup of $G$ such that $\Gamma \cap K \leq \Gamma_{(n)}$. Then $(\Gamma K / K)_{(n)}=\Gamma_{(n)} K / K$.

Proof of (a). Let $\mathcal{C}_{n}(\Gamma, N)$ be the set of all open subgroups $M$ of $\Gamma$ with $N \leq M$ and $(\Gamma: M) \leq n$. The map $M \mapsto M / N$ is a bijection of $\mathcal{C}_{n}(\Gamma, N)$ onto $\mathcal{C}_{n}(\Gamma / N, 1)$ that commutes with intersections. By definition, $\Gamma_{(n)}\left(\operatorname{resp} .(\Gamma / N)_{(n)}\right)$ is the intersection of all the groups in $\mathcal{C}_{n}(\Gamma, 1)$ (resp. 
$\left.\mathcal{C}_{n}(\Gamma / N, 1)\right)$. By assumption, $\mathcal{C}_{n}(\Gamma, 1)=\mathcal{C}_{n}\left(\Gamma, \Gamma_{(n)}\right)=\mathcal{C}_{n}(\Gamma, N)$. Therefore, $\Gamma_{(n)} / N=(\Gamma / N)_{(n)}$.

Proof of (b). By definition, $\mathcal{C}_{n}\left(\Gamma, N^{\prime}\right) \subseteq \mathcal{C}_{n}\left(\Gamma, \Gamma_{(n)}\right)$. By the proof of (a) and by assumption, $\left|\mathcal{C}_{n}\left(\Gamma, N^{\prime}\right)\right|=\left|\mathcal{C}_{n}\left(\Gamma / N^{\prime}, 1\right)\right|=\left|\mathcal{C}_{n}(\Gamma / N, 1)\right|=\left|\mathcal{C}_{n}(\Gamma, N)\right|$ $=\left|\mathcal{C}_{n}\left(\Gamma, \Gamma_{(n)}\right)\right|$. Hence, $\mathcal{C}_{n}\left(\Gamma, N^{\prime}\right)=\mathcal{C}_{n}\left(\Gamma, \Gamma_{(n)}\right)$, so $N^{\prime} \subseteq \bigcap_{M \in \mathcal{C}_{n}\left(\Gamma, N^{\prime}\right)} M=$ $\bigcap_{M \in \mathcal{C}_{n}\left(\Gamma, \Gamma_{(n)}\right)} M=\Gamma_{(n)}$.

Proof of (c). By (a), with $N=\Gamma \cap K=\Gamma_{(n)} \cap K$, we have $(\Gamma / \Gamma \cap$ $K)_{(n)}=\Gamma_{(n)} /(\Gamma \cap K)$. The isomorphism $\Gamma / \Gamma \cap K \rightarrow \Gamma K / K$ maps the left hand side onto $(\Gamma K / K)_{(n)}$ and the right hand side onto $\Gamma_{(n)} K / K$. Hence $(\Gamma K / K)_{(n)}=\Gamma_{(n)} K / K$.

LEMMA 1.2. Let $\Gamma$ be a finitely generated profinite group. Then for every $m \in \mathbb{N}$ there is an $n \geq m$ such that every automorphism of $\Gamma^{(m)}$ which lifts to an automorphism of $\Gamma^{(n)}$ lifts to an automorphism of $\Gamma$.

Proof. For each $n \geq m$ there are natural maps $\operatorname{Aut}(\Gamma) \rightarrow \operatorname{Aut}\left(\Gamma^{(n)}\right) \rightarrow$ $\operatorname{Aut}\left(\Gamma^{(m)}\right)$ of finite groups. Let $B_{n}$ be the image of $\operatorname{Aut}\left(\Gamma^{(n)}\right)$ in $\operatorname{Aut}\left(\Gamma^{(m)}\right)$. Then $\operatorname{Aut}\left(\Gamma^{(m)}\right)=B_{m} \geq B_{m+1} \geq B_{m+2} \geq \cdots$ and $\bigcap_{k=m}^{\infty} B_{k}$ is the image of $\operatorname{Aut}(\Gamma)$. Indeed, since $\bigcap_{k=1}^{\infty} \Gamma_{(k)}=1$, we have $\Gamma=\lim \Gamma^{(k)}$. If $\alpha \in \bigcap_{k=m}^{\infty} B_{k}$, then for each $k \geq m$ the inverse image of $\alpha$ in $\operatorname{Aut}\left(\overleftarrow{\Gamma^{(k)}}\right)$ is a nonempty finite set. The inverse limit of these images is nonempty [FrJ, Cor. 1.1.4]. Each sequence in the inverse limit gives rise to a lifting of $\alpha$ to an element of $\operatorname{Aut}(\Gamma)$. Since $B_{m}$ is finite, there is an $n \geq m$ such that $B_{n}=B_{n+1}=$ $B_{n+2}=\cdots$, hence $B_{n}$ is the image of $\operatorname{Aut}(\Gamma)$.

We will use the following reformulation of Lemma 1.2:

LEMma 1.3. Let $\Gamma$ and $\Delta$ be finitely generated isomorphic profinite groups. Then for each $m \in \mathbb{N}$ there is an $n \geq m$ with the following property: Let $N \leq K$ be open normal subgroups of $\Gamma$ and $M \leq L$ open normal subgroups of $\Delta$ such that

(a) $N \leq \Gamma_{(n)}$ or $M \leq \Delta_{(n)} ;$ and

(b) $\Gamma_{(m)} \leq K$ or $\Delta_{(m)} \leq L$.

Then every isomorphism $\Gamma / K \rightarrow \Delta / L$ that can be lifted to an isomorphism $\Gamma / N \rightarrow \Delta / M$ lifts to an isomorphism $\Gamma \rightarrow \Delta$. 
Proof. Each isomorphism $\Gamma \cong \Delta$ maps $\Gamma_{(m)}$ and $\Gamma_{(n)}$, respectively, onto $\Delta_{(m)}$ and $\Delta_{(n)}$. Thus, we may assume that $\Delta=\Gamma$. For $m \in \mathbb{N}$ we choose $n \geq m$ as in Lemma 1.2. Let $K, L, M, N$ be groups satisfying (a) and (b) as in the Lemma. We may assume that there is an isomorphism $\Gamma / K \cong \Gamma / L$ and an isomorphism $\alpha: \Gamma / N \rightarrow \Gamma / M$ which maps $K / N$ onto $L / M$. Hence, $(\Gamma / N: K / N)=(\Gamma / M: L / M)$.

Under these assumptions we may strengthen (a) and (b) to

$\left(\mathrm{a}^{\prime}\right) \quad N \leq \Gamma_{(n)}$ and $M \leq \Gamma_{(n)} ; \quad$ and

$\left(\mathrm{b}^{\prime}\right) \Gamma_{(m)} \leq K$ and $\Gamma_{(m)} \leq L$.

Indeed, the isomorphism $\Gamma / N \cong \Gamma / M$ and Lemma 1.1(b) imply that $N \leq \Gamma_{(n)}$ if and only if $M \leq \Gamma_{(n)}$. This proves $\left(\mathrm{a}^{\prime}\right)$. In particular, $N \leq \Gamma_{(m)}$ and $M \leq \Gamma_{(m)}$.

The existence of $\alpha$ implies that $(\Gamma / N)_{(m)} \leq K / N$ if and only if $(\Gamma / M)_{(m)} \leq L / M$. By Lemma 1.1(a), $\Gamma_{(m)} / N \leq K / N$ if and only if $\Gamma_{(m)} / M \leq L / M$, that is, $\Gamma_{(m)} \leq K$ if and only if $\Gamma_{(m)} \leq L$. This proves $\left(\mathrm{b}^{\prime}\right)$.

Now let $\theta: \Gamma / N \rightarrow \Gamma / M$ be an isomorphism which induces an isomorphism $\theta^{\prime}: \Gamma / K \rightarrow \Gamma / L$. Thus we have the following commutative diagram

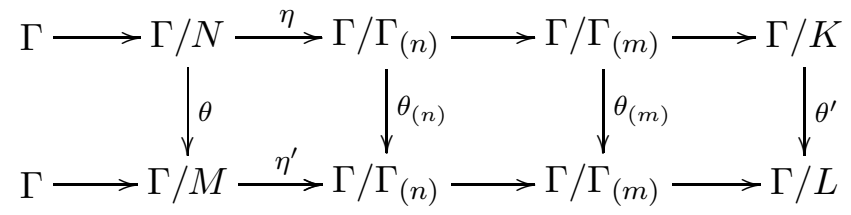

in which the horizontal maps are the quotient maps (and $\theta_{(n)}$ and $\theta_{(m)}$ are constructed below).

By Lemma 1.1(a), $\operatorname{Ker}(\eta)=\Gamma_{(n)} / N=(\Gamma / N)_{(n)} ;$ similarly $\operatorname{Ker}\left(\eta^{\prime}\right)=$ $(\Gamma / M)_{(n)}$. Therefore $\theta$ induces an automorphism $\theta_{(n)}$ of $\Gamma^{(n)}=\Gamma / \Gamma_{(n)}$ making the above diagram commutative. The automorphism $\theta_{(m)}$ of $\Gamma^{(m)}=$ $\Gamma / \Gamma_{(m)}$ is constructed similarly.

By Lemma $1.2, \theta_{(m)}$, hence also $\theta^{\prime}$, lifts to an automorphism of $\Gamma$.

\section{$\S 2$. Topologies}

Let $G$ be a profinite group. The set $\operatorname{Subgr}(G)$ of all closed subsets of $G$ has two natural topologies. A basis for the first topology consists of all subsets $\mathcal{U}\left(G_{1}, N\right)=\left\{H \in \operatorname{Subgr}(G) \mid H N=G_{1} N\right\}$, where $G_{1} \in \operatorname{Subgr}(G)$ 
and $N$ is an open normal subgroup of $G$. This topology is referred to in [HJPa] and $[\mathrm{HJPb}]$ as the strict topology. If $G$ is finite, the strict topology of $\operatorname{Subgr}(G)$ coincides with its discrete topology. In the general case, $\operatorname{Subgr}(G)=\lim \operatorname{Subgr}(G / N)$, where $N$ ranges over all open normal subgroups of $G$. Thus, $\operatorname{Subgr}(G)$ is a profinite space under the strict topology. In particular, a subset of $\operatorname{Subgr}(G)$ is strictly closed if and only if it is strictly compact.

In addition to the strict topology, $\operatorname{Subgr}(G)$ admits a weaker topology, called the étale topology, which is in general not Hausdorff. A basis for the étale topology consists of all the subsets $\operatorname{Subgr}(M)$ with $M$ open in $G$. Thus, each étale open (closed) subset of $\operatorname{Subgr}(G)$ is strictly open (closed), and each strictly closed subset of $\operatorname{Subgr}(G)$ is strictly compact, hence étale compact.

We mainly use the strict topology, so we usually drop the reference to it.

Lemma 2.1. Let $G$ be a profinite group and $\mathcal{G}$ a closed subset of $\operatorname{Subgr}(G)$. Suppose there are no inclusions between distinct groups in $\mathcal{G}$. Then the étale topology of $\mathcal{G}$ coincides with its strict topology.

Proof. Since the strict topology of $\operatorname{Subgr}(G)$ is finer than its étale topology, it suffices to prove for each $G_{1} \in \mathcal{G}$ that each basic strictly open neighborhood $\mathcal{N}_{1}$ of $G_{1}$ in $\mathcal{G}$ contains an étale open neighborhood of $G_{1}$. In fact, $\mathcal{N}_{1}=\left\{H \in \mathcal{G} \mid H N=G_{1} N\right\}$ for some an open normal subgroup $N$ of $G$. Let $\mathcal{M}$ be the set of all open subgroups $M$ of $G$ with $G_{1} \leq M \leq G_{1} N$. It suffices to find $M \in \mathcal{M}$ such that $\operatorname{Subgr}(M) \subseteq \mathcal{N}_{1}$.

Assume that such $M$ does not exists. Then, for each $M \in \mathcal{M}$

$$
\mathcal{H}_{M}=\left\{H \in \mathcal{G} \mid H \leq M, H N \neq G_{1} N\right\} \neq \emptyset .
$$

The set $\mathcal{H}_{M}$ is the intersection of $\mathcal{G}$ with two strictly closed subsets

$$
\{H \in \operatorname{Subgr}(G) \mid H \leq M\} \quad \text { and } \quad\left\{H \in \operatorname{Subgr}(G) \mid H N \neq G_{1} N\right\}
$$

of $\operatorname{Subgr}(G)$, so $\mathcal{H}_{M}$ is strictly closed in $\mathcal{G}$. For all $M_{1}, \ldots, M_{n} \in \mathcal{M}$ we have,

$$
\mathcal{H}_{M_{1} \cap \cdots \cap M_{n}} \subseteq \mathcal{H}_{M_{1}} \cap \cdots \cap \mathcal{H}_{M_{n}}
$$

and $M_{1} \cap \cdots \cap M_{n} \in \mathcal{M}$. Hence $\mathcal{H}_{M_{1}} \cap \cdots \cap \mathcal{H}_{M_{n}} \neq \emptyset$. Since $\mathcal{G}$ is strictly closed, $\mathcal{G}$ is strictly compact. Therefore, there exists $H \in \bigcap_{M \in \mathcal{M}} \mathcal{H}_{M}$. In particular, $H \in \mathcal{G}, H N \neq G_{1} N$, and $H \leq G_{1}$. By assumption, $H=G_{1}$. This leads to the contradiction $G_{1} N \neq G_{1} N$. 
For a profinite group $G$ and closed subgroup $H_{1}, H_{2}$ we consider the space of the double cosets $H_{1} \backslash G / H_{2}$ with its quotient topology.

Lemma 2.2. Let $G$ be a profinite group, $g \in G$, and $H_{1}, H_{2}$ closed subgroups of $G$. Then $H_{1} g H_{2}$ is an isolated point in the quotient space $H_{1} \backslash G / H_{2}$ if and only if $H_{1}^{g} H_{2}$ is open in $G$.

Proof. By the definition of the quotient topology, the point $H_{1} g H_{2} \in$ $H_{1} \backslash G / H_{2}$ is isolated if and only if its preimage $H_{1} g H_{2}$ in $G$ is open. Since multiplication from the left by $g^{-1}$ is a homeomorphism of $G$, this is equivalent to $H_{1}^{g} H_{2}$ being open in $G$.

\section{§3. Group piles}

One of the main objects in $[\mathrm{HJPa}]$ and $[\mathrm{HJPb}]$ is a group structure. A group structure is defined to be data $\left(X, G, G_{x}\right)_{x \in S}$ consisting of a profinite space $X$, a profinite group $G$, and a closed subgroup $G_{x}$ of $G$ for each $x \in X$, satisfying certain conditions. Among others, $G$ acts continuously on $X$ from the right such that $G_{x^{\sigma}}=G_{x}^{\sigma}$ for all $\sigma \in G$ and $x \in X$ and the stabilizer of each $x$ is contained in $G_{x}$. In this work we omit $X$, retain the profinite group $G$ and the collection $\mathcal{G}=\left\{G_{x} \mid x \in X\right\}$, relax the conditions imposed on the group structure, and call the structure obtained in this way a "group pile".

The group $G$ acts continuously on $\operatorname{Subgr}(G)$ by conjugation from the right. A $G$-domain of $\operatorname{Subgr}(G)$ is a subset of $\operatorname{Subgr}(G)$ closed under that action. In particular, each conjugacy domain $\left\{G_{0}^{g} \mid g \in G\right\}$ with a closed subgroup $G_{0}$ of $G$ is a closed $G$-domain which we call a $G$-class.

We fix a finite set $S$ containing 0 but not containing 1 and set $S_{0}=\{0\}$ and $S_{1}=S \backslash\{0\}$.

A group pile is a structure $\mathbf{G}=\left(G, \mathcal{G}_{v}\right)_{v \in S}$ consisting of a profinite group $G$, a $G$-class $\mathcal{G}_{0}$ and a closed $G$-domain $\mathcal{G}_{v}$ of $\operatorname{Subgr}(G)$ for each $v \in S_{1}$. We set $\mathcal{G}=\bigcup_{v \in S} \mathcal{G}_{v}, \mathcal{G}_{1}=\bigcup_{v \in S_{1}} \mathcal{G}_{v}$, and assume that each $H \in \mathcal{G}$ is finitely generated.

We call $\mathbf{G}$ finite if $G$ is finite. We say that $\mathbf{G}$ is self-generated if there exists $G_{0} \in \mathcal{G}_{0}$ such that $G=\left\langle G_{0}, \mathcal{G}_{1}\right\rangle=\left\langle G_{0}, G_{1} \mid G_{1} \in \mathcal{G}_{1}\right\rangle$. We call $\mathbf{G}$ separated if the $\mathcal{G}_{v}$ 's are disjoint. Thus $\mathcal{G}=\bigcup_{v \in S} \mathcal{G}_{v}$ is a partition into open-closed sets. An epimorphism $\varphi: \mathbf{G} \rightarrow \mathbf{A}=\left(A, \mathcal{A}_{0}, \mathcal{A}_{1}\right)$ of group piles is an epimorphism of profinite groups $\varphi: G \rightarrow A$ such that $\varphi\left(\mathcal{G}_{v}\right)=\mathcal{A}_{v}$ for each $v \in S$. It is an isomorphism, if $\varphi: G \rightarrow A$ is an isomorphism 
of groups; equivalently, $\varphi: \mathbf{G} \rightarrow \mathbf{A}$ has an inverse. Each epimorphism $\varphi: \mathbf{G} \rightarrow \mathbf{A}$ is determined, up to an isomorphism, by $\operatorname{Ker}(\varphi)$. If $\mathbf{G}$ is selfgenerated, so is $\mathbf{A}$. If $\mathbf{A}$ is separated, so is $\mathbf{G}$.

We say that $\mathbf{G}$ is deficient if $\mathcal{G}_{0}$ consists of the trivial subgroup of $G$. In this case we omit $\mathcal{G}_{0}$ from $\mathbf{G}$ and rewrite it also as $\left(G, \mathcal{G}_{v}\right)_{v \in S_{1}}$. Note that if $\varphi: \mathbf{G} \rightarrow \mathbf{A}$ is an epimorphism of group piles and $\mathbf{G}$ is deficient, then so is $\mathbf{A}$. Likewise, in this case, each of the assumptions about $\mathcal{G}_{0}$ done in the forthcoming definitions and all statements about $\mathcal{G}_{0}$ hold trivially. We will mention that in the sequel only occasionally.

Let $\mathbf{A}=\left(A, \mathcal{A}_{v}\right)_{v \in S}$ be a finite group pile and $\varphi: \mathbf{G} \rightarrow \mathbf{A}$ an epimorphism. A decomposition of $\varphi$ is a pair of epimorphisms $\hat{\varphi}: \mathbf{G} \rightarrow \hat{\mathbf{A}}$ and $\bar{\varphi}: \hat{\mathbf{A}} \rightarrow \mathbf{A}$, where $\hat{\mathbf{A}}=\left(\hat{A}, \hat{\mathcal{A}}_{v}\right)_{v \in S}$ is a finite group pile and $\bar{\varphi} \circ \hat{\varphi}=\varphi$. The kernel of the decomposition $\operatorname{Ker}(\hat{\varphi})$ is an open normal subgroup of $G$ contained in $\operatorname{Ker}(\varphi)$. Conversely, for each open normal subgroup $K$ of $G$ contained in $\operatorname{Ker}(\varphi)$ there is a decomposition of $\varphi$ with kernel $K$, unique up to an isomorphism. Namely, let $\hat{A}=G / K$ and let $\hat{\varphi}: G \rightarrow \hat{A}$ be the quotient map $G \rightarrow G / K$. Put $\hat{\mathcal{A}}_{v}=\hat{\varphi}\left(\mathcal{G}_{v}\right)$ and $\hat{\mathbf{A}}=\left(\hat{A}, \hat{\mathcal{A}}_{v}\right)_{v \in S}$. Then the induced epimorphism of groups $\bar{\varphi}: \hat{A} \rightarrow A$ maps $\hat{\mathcal{A}}_{v}$ onto $\mathcal{A}_{v}$ for each $v \in V$, so it is an epimorphism $\bar{\varphi}: \hat{\mathbf{A}} \rightarrow \mathbf{A}$ of group piles.

Lemma 3.1. Let $\mathbf{G}=\left(G, \mathcal{G}_{v}\right)_{v \in S}$ be a separated group pile. Suppose that for each $v \in S$ there is a finitely generated group $\Gamma_{v}$ with $G_{v} \cong \Gamma_{v}$ for every $G_{v} \in \mathcal{G}_{v}$.

(a) Let $n \in \mathbb{N}$. Then there exists an open normal subgroup $K$ of $G$ such that, in the notation of Section $1, H \cap K \leq H_{(n)}$ for every $H \in \mathcal{G}$.

(b) There is an open normal subgroup $K$ of $G$ such that if $\varphi: \mathbf{G} \rightarrow \mathbf{A}$ is an epimorphism of group piles with $\operatorname{Ker}(\varphi) \leq K$, then $\mathbf{A}$ is separated.

Proof of (a). Consider $H \in \mathcal{G}$ and let $v$ be the unique index with $H \in$ $\mathcal{G}_{v}$. Then $H \cong \Gamma_{v}$, so $H$ is finitely generated. In addition, $\mathcal{G}_{v}$ is open in $\mathcal{G}$. Therefore, there is an open normal subgroup $K$ of $G$ such that $H \cap K \leq H_{(n)}$ and if $H^{\prime} \in \mathcal{G}$ satisfies $H^{\prime} K=H K$, then $H^{\prime} \in \mathcal{G}_{v}$, hence $H^{\prime} \cong \Gamma_{v} \cong H$. The equality $H K=H^{\prime} K$ implies $H / H \cap K \cong H^{\prime} / H^{\prime} \cap K$. The isomorphism $H \cong H^{\prime}$ implies $\left(H: H_{(n)}\right)=\left(H^{\prime}: H_{(n)}^{\prime}\right)$. Hence

$$
\left(H^{\prime}: H^{\prime} \cap K\right)=(H: H \cap K) \geq\left(H: H_{(n)}\right)=\left(H^{\prime}: H_{(n)}^{\prime}\right) .
$$

By Lemma 1.1(b), $H^{\prime} \cap K \leq H_{(n)}^{\prime}$. 
Finally, since $\mathcal{G}$ is compact, we may choose $K$ to be independent of $H$.

Proof of (b). By compactness each of the sets $\mathcal{G}_{v}$ is the finite union of sets of the form $\left\{H \in \operatorname{Subgr}(G) \mid H K_{k}=M_{k}\right\}$, where $K_{k}$ are open normal subgroups of $G$ and $M_{k}$ are open subgroups of $G$. The intersection $K$ of all of the $K_{k}$ 's has the required property.

Indeed, let $\varphi: \mathbf{G} \rightarrow \mathbf{A}$ be an epimorphism with $\operatorname{Ker}(\varphi) \leq K$, let $v, v^{\prime} \in$ $S$ be distinct, and let $G_{v} \in \mathcal{G}_{v}$ and $G_{v^{\prime}} \in \mathcal{G}_{v^{\prime}}$. There is a $k$ such that $G_{v^{\prime}} K_{k}=M_{k}$. Since $G_{v} \notin \mathcal{G}_{v^{\prime}}$, we have $G_{v} K_{k} \neq M_{k}$, so $G_{v} K_{k} \neq G_{v^{\prime}} K_{k}$. Since $\operatorname{Ker}(\varphi) \leq K \leq K_{k}$, this implies $G_{v} \operatorname{Ker}(\varphi) \neq G_{v^{\prime}} \operatorname{Ker}(\varphi)$, so $\varphi\left(G_{v}\right) \neq$ $\varphi\left(G_{v^{\prime}}\right)$. Thus, in the notation introduced prior to the lemma, $\hat{\mathcal{A}}_{v}$ is disjoint from $\hat{\mathcal{A}}_{v^{\prime}}$, which means that $\mathbf{A}$ is separated.

Lemma 3.2. Let $\mathbf{G}=\left(G, \mathcal{G}_{v}\right)_{v \in S}$ be a group pile. Suppose there are no inclusions between distinct groups in $\mathcal{G}_{1}=\bigcup_{v \in S_{1}} \mathcal{G}_{v}$. Let $\varphi: \mathbf{G} \rightarrow \mathbf{A}$ be an epimorphism onto a finite group pile $\mathbf{A}$. Then there is an open normal subgroup $N$ of $G$ with the following property: Let $(\hat{\varphi}, \bar{\varphi})$ be a decomposition of $\varphi$ with $\operatorname{Ker}(\hat{\varphi}) \leq N$. If $G_{1}, G_{2} \in \mathcal{G}_{1}$ and $\hat{\varphi}\left(G_{1}\right) \leq \hat{\varphi}\left(G_{2}\right)$, then $\varphi\left(G_{1}\right)=$ $\varphi\left(G_{2}\right)$.

Proof. Let $A_{1}, A_{2} \in \mathcal{A}_{1}=\varphi\left(\mathcal{G}_{1}\right)$ such that $A_{1} \neq A_{2}$. Consider the compact subsets $\mathcal{G}^{(1)}=\left\{G_{1} \in \mathcal{G}_{1} \mid \varphi\left(G_{1}\right)=A_{1}\right\}$ and $\mathcal{G}^{(2)}=\left\{G_{2} \in \mathcal{G}_{1} \mid\right.$ $\left.\varphi\left(G_{2}\right)=A_{2}\right\}$ of $\mathcal{G}_{1}$. Let $G_{1} \in \mathcal{G}^{(1)}, G_{2} \in \mathcal{G}^{(2)}$. Then $G_{1} \neq G_{2}$, so $G_{1} \not \leq G_{2}$. Therefore there is an open normal subgroup $N=N\left(G_{1}, G_{2}\right)$ of $G$ such that $G_{1} N \not \leq G_{2} N$. If $G_{1}^{\prime} \in \mathcal{G}^{(i)}$ satisfies $G_{1}^{\prime} N=G_{1} N$ and $G_{2}^{\prime} \in \mathcal{G}^{(i)}$ satisfies $G_{2}^{\prime} N=G_{2} N$, then $G_{1}^{\prime} N \not \leq G_{2}^{\prime} N$. By the compactness of $\mathcal{G}^{(1)} \times \mathcal{G}^{(2)}$, there is an open normal subgroup $N$ of $G$, such that $G_{1} N \not \leq G_{2} N$ for all $G_{1} \in \mathcal{G}^{(1)}, G_{2} \in \mathcal{G}^{(2)}$. This remains true if we replace $N$ by any open normal subgroup $K$ of $G$ contained in $N$. Thus, since $\mathcal{A}$ is finite, we may assume that $N$ is good for all $A_{1} \neq A_{2}$ in $\mathcal{A}$. Consequently, if $\hat{\varphi}: \mathbf{G} \rightarrow \hat{\mathbf{A}}$ is an epimorphism with $\operatorname{Ker}(\hat{\varphi}) \leq N$, and $G_{1}, G_{2} \in \mathcal{G}_{1}$ satisfy $\varphi\left(G_{1}\right) \neq \varphi\left(G_{2}\right)$, then $\hat{\varphi}\left(G_{1}\right) \not \leq \hat{\varphi}\left(G_{2}\right)$.

\section{§4. Embedding problems for group piles}

We show how to dominate locally solvable embedding problems for groups piles with rigid finite embedding problems having extra properties.

First we introduce an appropriate vocabulary. Let $\mathbf{G}=\left(G, \mathcal{G}_{v}\right)_{v \in S}$ and $\mathbf{A}=\left(A, \mathcal{A}_{v}\right)_{v \in S}$ be group piles. An epimorphism $\varphi: \mathbf{G} \rightarrow \mathbf{A}$ is rigid if $\varphi: G \rightarrow A$ is injective on each $G^{\prime} \in \mathcal{G}$. 
An embedding problem for $\mathbf{G}$ is a pair of epimorphisms of group piles

$$
(\varphi: \mathbf{G} \rightarrow \mathbf{A}, \alpha: \mathbf{B} \rightarrow \mathbf{A})
$$

which we eventually abbreviate as $(\varphi, \alpha)$. We say that (1) is rigid, if $\alpha$ is rigid, that is, $\alpha: B \rightarrow A$ is injective on each $B^{\prime} \in \mathcal{B}$. We say that (1) is finite, if $B$ is finite. We say that (1) splits group theoretically if there exists a group homomorphism $\alpha^{\prime}: A \rightarrow B$ satisfying $\alpha \circ \alpha^{\prime}=\mathrm{id}_{A}$. We say that (1) is self-generated, if $\mathbf{G}, \mathbf{A}, \mathbf{B}$ are self-generated.

Groups $B^{\prime} \in \mathcal{B}$ and $G^{\prime} \in \mathcal{G}$ are compatible for the embedding problem (1) if there exists an epimorphism $\gamma^{\prime}: G^{\prime} \rightarrow B^{\prime}$ such that $\alpha \circ \gamma^{\prime}=$ $\left.\varphi\right|_{G^{\prime}}$; in particular, $\varphi\left(G^{\prime}\right)=\alpha\left(B^{\prime}\right)$. Note that if $B^{\prime}$ and $G^{\prime}$ are compatible and $B^{\prime \prime}$ is conjugate to $B^{\prime}$, then there is a conjugate $G^{\prime \prime}$ of $G^{\prime}$ which is compatible with $B^{\prime \prime}$. Indeed, if $B^{\prime \prime}=\left(B^{\prime}\right)^{b}$, choose $g \in G$ such that $\varphi(g)=$ $\alpha(b)$. Then $\left(G^{\prime}\right)^{g}$ is compatible with $B^{\prime}$. Similarly, if $G^{\prime \prime}$ is conjugate to $G^{\prime}$, then $G^{\prime \prime}$ is compatible with some conjugate of $B^{\prime}$.

We say that (1) is locally solvable if the following holds for each $v \in S$ :

(2a) for every $B_{v} \in \mathcal{B}_{v}$ there exists a compatible $G_{v} \in \mathcal{G}_{v}$,

(2b) for every $G_{v} \in \mathcal{G}_{v}$ there exists a compatible $B_{v} \in \mathcal{B}_{v}$.

Note that it suffices to demand (2a) only for every $B_{v}$ in a system of representatives of the $B$-classes of $\mathcal{B}_{v}$. Similarly it suffices to demand $(2 \mathrm{~b})$ only for a system of representatives of the $G$-classes of $\mathcal{G}_{v}$.

If both $\mathbf{G}$ and $\mathbf{B}$ in (1) are deficient, then for (1) to be locally solvable it suffices that (2) holds only for $v \in S_{1}$.

A rigid embedding problem is always locally solvable. A solution of (1) is an epimorphism $\gamma: \mathbf{G} \rightarrow \mathbf{B}$ such that $\alpha \circ \gamma=\varphi$.

Let (1) be an embedding problem. Another embedding problem

$$
(\hat{\varphi}: \mathbf{G} \rightarrow \hat{\mathbf{A}}, \hat{\alpha}: \hat{\mathbf{B}} \rightarrow \hat{\mathbf{A}})
$$

is said to dominate (1) if there exists a commutative diagram

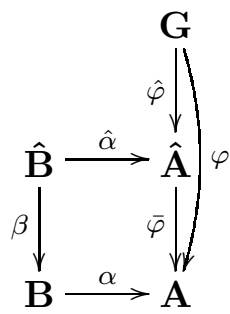


of epimorphisms of group piles. If $\hat{\gamma}: \mathbf{G} \rightarrow \hat{\mathbf{B}}$ is a solution of $(3)$, then $\beta \circ \hat{\gamma}$ is a solution of (1).

If $\hat{B}$ is a subgroup of the fiber product $B \times_{A} \hat{A}$ [FrJ, Sec. 22.2], $\beta$ and $\hat{\alpha}$ are the restrictions of the projections on the corresponding components, and both $\mathbf{B}$ and $\mathbf{G}$ are deficient, then so is $\hat{\mathbf{A}}$. In this case, $\beta\left(\hat{B}_{0}\right)=1$ and $\hat{\alpha}\left(\hat{B}_{0}\right)=1$, so $\hat{B}_{0}=1$ for each $\hat{B}_{0} \in \hat{\mathcal{B}}_{0}$. Therefore, $\hat{\mathbf{B}}$ is deficient. We will often use this observation without mentioning it.

The following result replaces $\mathbf{G}$ in a locally solvable embedding problem (1) by a finite group pile.

LEMMA 4.1. Let (1) be a finite locally solvable embedding problem. Then there exists an open normal subgroup $N$ of $G$ with the following property. Let $\mathbf{G} \stackrel{\hat{\varphi}}{\longrightarrow} \hat{\mathbf{A}} \stackrel{\bar{\varphi}}{\longrightarrow} \mathbf{A}$ be a decomposition of $\varphi$ with $\operatorname{Ker}(\hat{\varphi}) \leq N$. Then $(\bar{\varphi}: \hat{\mathbf{A}} \rightarrow \mathbf{A}, \alpha: \mathbf{B} \rightarrow \mathbf{A})$ is a finite locally solvable embedding problem.

Proof. Let $v \in S$ and consider the family $\left\{\left(B_{i}, G_{i}\right) \in \mathcal{B} \times \mathcal{G} \mid i \in I_{v}\right\}$ of all compatible pairs for (1) with $B_{i} \in \mathcal{B}_{v}$ and $G_{i} \in \mathcal{G}_{v}$. For each $i \in I_{v}$ choose an epimorphism $\gamma_{i}: G_{i} \rightarrow B_{i}$ with $\alpha \circ \gamma_{i}=\left.\varphi\right|_{G_{i}}$. Then $\operatorname{Ker}\left(\gamma_{i}\right)$ is an open subgroup of $G_{i}$. Choose an open normal subgroup $N_{i}$ of $G$ satisfying $N_{i} \leq \operatorname{Ker}(\varphi)$ and $G_{i} \cap N_{i} \leq \operatorname{Ker}\left(\gamma_{i}\right)$. Then $\mathcal{G}^{(i)}=\left\{G^{\prime} \in \mathcal{G}_{v} \mid G^{\prime} N_{i}=G_{i} N_{i}\right\}$ is an open-closed neighborhood of $G_{i}$ in $\mathcal{G}_{v}$ and $\gamma_{i}$ extends to an epimorphism $\delta_{i}: G_{i} N_{i} \rightarrow B_{i}$ with kernel $\operatorname{Ker}\left(\gamma_{i}\right) N_{i}$ such that $\alpha \circ \delta_{i}=\left.\varphi\right|_{G_{i} N_{i}}$.

Since (1) is locally solvable, $\mathcal{B}_{v}=\left\{B_{i} \in \mathcal{B} \mid i \in I_{v}\right\}$ and $\mathcal{G}_{v}=\left\{G_{i} \in\right.$ $\left.\mathcal{G} \mid i \in I_{v}\right\}$, so $\mathcal{G}_{v}=\bigcup_{i \in I_{v}} \mathcal{G}^{(i)}$. Since $\mathcal{G}_{v}$ is compact, there is a finite subset $J_{v}$ of $I_{v}$ such that $\mathcal{G}_{v}=\bigcup_{i \in J_{v}} \mathcal{G}^{(i)}$. Add more elements to $J_{v}$, if necessary, to get $\mathcal{B}_{v}=\left\{B_{i} \in \mathcal{B} \mid i \in J_{v}\right\}$ and put $N_{v}=\bigcap_{i \in J_{v}} N_{i}$. Let $N=\bigcap_{v \in S} N_{v}$. Then $N$ is an open normal subgroup of $G$.

Now let $v \in S$ and consider a decomposition $\mathbf{G} \stackrel{\hat{\varphi}}{\longrightarrow} \hat{\mathbf{A}} \stackrel{\bar{\varphi}}{\longrightarrow} \mathbf{A}$ of $\varphi$ with $\operatorname{Ker}(\hat{\varphi}) \leq N$. Let $\hat{A}^{\prime} \in \hat{\mathcal{A}}_{v}$. There are $i \in J_{v}$ and $G^{\prime} \in \mathcal{G}^{(i)} \subseteq \mathcal{G}_{v}$ such that $\hat{A}^{\prime}=\hat{\varphi}\left(G^{\prime}\right)$. Let $\hat{\varphi}^{\prime}: G^{\prime} \rightarrow \hat{A}^{\prime}$ and $\delta_{i}^{\prime}: G^{\prime} \rightarrow B$ be the restrictions of $\hat{\varphi}$ and $\delta_{i}$ from $G$ and $G_{i} N_{i}$, respectively, to $G^{\prime}$. Since $G^{\prime} N_{i}=G_{i} N_{i}$ and $\operatorname{Ker}(\hat{\varphi}) \leq N_{i} \leq \operatorname{Ker}\left(\delta_{i}\right)$, we have $\delta_{i}^{\prime}\left(G^{\prime}\right)=\delta_{i}\left(G_{i}\right)=B_{i} \in \mathcal{B}_{v}$ and $\delta_{i}^{\prime}$ induces an epimorphism $\bar{\delta}^{\prime}: \hat{A}^{\prime} \rightarrow B_{i}$ such that $\bar{\delta}^{\prime} \circ \hat{\varphi}^{\prime}=\delta_{i}^{\prime}$. Hence, $\alpha \circ \bar{\delta}^{\prime} \circ \hat{\varphi}^{\prime}=\alpha \circ \delta_{i}^{\prime}=\left.\varphi\right|_{G^{\prime}}=\bar{\varphi} \circ \hat{\varphi}^{\prime}$. Since $\hat{\varphi}^{\prime}: G^{\prime} \rightarrow \hat{A}^{\prime}$ is an epimorphism, $\alpha \circ \bar{\delta}^{\prime}=\left.\bar{\varphi}\right|_{\hat{A}^{\prime}}$. Thus, $\left(B_{i}, \hat{A}^{\prime}\right)$ is a compatible pair.

Conversely, let $B^{\prime} \in \mathcal{B}_{v}$. Then $B^{\prime}=B_{i}$ for some $i \in J_{v}$. Set $\hat{A}_{i}=\hat{\varphi}\left(G_{i}\right)$. Then $\hat{A}_{i} \in \hat{\mathcal{A}}_{v}$ and $\left(B_{i}, \hat{A}_{i}\right)$ is a compatible pair. 
The following construction will be used several times to produce dominating embedding problems.

LEMma-ConstruCtion 4.2. Let (1) be a finite embedding problem and

$$
\mathbf{G} \stackrel{\hat{\varphi}}{\longrightarrow} \hat{\mathbf{A}} \stackrel{\bar{\varphi}}{\longrightarrow} \mathbf{A}
$$

a decomposition of $\varphi$. Let $I_{v}, v \in S$, be disjoint sets such that $I_{0}=\{0\}$. For each $i \in I_{v}$ let $\left(B_{i}, \hat{A}_{i}\right) \in \mathcal{B}_{v} \times \hat{\mathcal{A}}_{v}$ be a compatible pair for the embedding problem $(\bar{\varphi}: \hat{A} \rightarrow A, \alpha: \mathbf{B} \rightarrow \mathbf{A})$ such that

$$
\left\{B_{i}^{b} \mid i \in I_{v}, b \in B\right\}=\mathcal{B}_{v} \quad \text { and } \quad\left\{\hat{A}_{i}^{\hat{a}} \mid i \in I_{v}, \hat{a} \in \hat{A}\right\}=\hat{\mathcal{A}}_{v} .
$$

Set $\hat{B}=B \times_{A} \hat{A}$ with the coordinate projections $\hat{\alpha}: \hat{B} \rightarrow \hat{A}$ and $\beta: \hat{B} \rightarrow B$. For each $i \in I_{v}$ let $\gamma_{i}: \hat{A}_{i} \rightarrow B_{i}$ be an epimorphism such that $\alpha \circ \gamma_{i}=\left.\bar{\varphi}\right|_{\hat{A}_{i}}$. It defines a homomorphism $\hat{\gamma}_{i}: \hat{A}_{i} \rightarrow \hat{B}$ such that

$$
\beta \circ \hat{\gamma}_{i}=\gamma_{i} \quad \text { and } \quad \hat{\alpha} \circ \hat{\gamma}_{i}=\operatorname{id}_{\hat{A}_{i}} .
$$

Let $\hat{B}_{i}=\hat{\gamma}_{i}\left(\hat{A}_{i}\right), \hat{\mathcal{B}}_{v}=\left\{\hat{B}_{i}^{\hat{b}} \mid i \in I_{v}, \hat{b} \in \hat{B}\right\}$, and $\hat{\mathbf{B}}=\left(\hat{B}, \hat{\mathcal{B}}_{v}\right)_{v \in S}$. Then:

(a) $\hat{\mathbf{B}}$ is a group pile and (3) is a finite rigid embedding problem that dominates (1).

(b) Suppose $\left\{\hat{A}_{i} \mid i \in I_{v}\right\}$ is a set of representatives of the $\hat{A}$-classes of $\hat{\mathcal{A}}_{v}$. Let $i \in I_{v}$ and $\hat{B}^{\prime} \in \hat{\mathcal{B}}_{v}$. If $\hat{\alpha}\left(\hat{B}^{\prime}\right)$ is conjugate in $\hat{A}$ to $\hat{A}_{i}$, then $\beta\left(\hat{B}^{\prime}\right)$ is conjugate in $B$ to $B_{i}$.

(c) If $\mathbf{B}$ and $\hat{\mathbf{A}}$ are deficient, then so is $\hat{\mathbf{B}}$.

Proof of (a). For each $i \in I_{v}$ we have, $\beta\left(\hat{B}_{i}\right)=\beta \circ \hat{\gamma}_{i}\left(\hat{A}_{i}\right)=\gamma_{i}\left(\hat{A}_{i}\right)=B_{i}$ and $\hat{\alpha}\left(\hat{B}_{i}\right)=\alpha \circ \hat{\gamma}_{i}\left(\hat{A}_{i}\right)=\hat{A}_{i}$ (by $\left.(6)\right)$. Hence, by $(5), \beta\left(\hat{\mathcal{B}}_{v}\right)=\mathcal{B}_{v}$ and $\hat{\alpha}\left(\hat{\mathcal{B}}_{v}\right)=\hat{A}_{v}$. Thus, $\beta: \hat{\mathbf{B}} \rightarrow \mathbf{B}$ and $\hat{\alpha}: \hat{\mathbf{B}} \rightarrow \hat{\mathbf{A}}$ are epimorphisms. Let $i \in I$. Since $\hat{\alpha} \circ \hat{\gamma}_{i}=\operatorname{id}_{\hat{A}_{i}}$, the restriction of $\hat{\alpha}$ to $\hat{B}_{i}$ is an isomorphism onto $\hat{A}_{i}$. By conjugation, $\hat{\alpha}$ is injective on each group in $\hat{\mathcal{B}}_{v}$. Since the latter consequence holds for each $v \in S$, the map $\hat{\alpha}$ is injective on each group in $\hat{\mathcal{B}}$.

Proof of (b). There exist $j \in I_{v}$ and $\hat{b} \in \hat{B}$ such that $\hat{B}^{\prime}=\hat{B}_{j}^{\hat{b}}$. Thus, $\hat{\alpha}\left(\hat{B}^{\prime}\right)=\hat{A}_{j}^{\hat{\alpha}(\hat{b})}$ is conjugate to $\hat{A}_{j}$. On the other hand, by assumption, $\hat{\alpha}\left(\hat{B}^{\prime}\right)$ is conjugate to $\hat{A}_{i}$. Hence, $j=i$, so $\beta\left(\hat{B}^{\prime}\right)=B_{j}^{\beta(\hat{b})}$ is conjugate to $B_{j}=B_{i}$. 
Recall that $G$ is said to be $\mathcal{G}_{1}$-projective if for every finite embedding problem $(\varphi: G \rightarrow A, \alpha: B \rightarrow A)$ satisfying

(7) for each $\Gamma \in \mathcal{G}_{1}$ there exists a homomorphism $\gamma_{\Gamma}: \Gamma \rightarrow B$ with $\alpha \circ \gamma_{\Gamma}=$ $\left.\varphi\right|_{\Gamma}$

there exists a homomorphism $\gamma: G \rightarrow B$ such that $\alpha \circ \gamma=\varphi$ [HJPb, Sec. 3].

The following result is a variant of [HJPb, Lemma 3.1].

LEMMA 4.3. Let (1) be a finite locally solvable embedding problem. Then there exists an open normal subgroup $N$ of $G$ with the following property: Let $K$ be an open normal subgroup of $G$ contained in $N$. Then (1) can be dominated by a finite rigid embedding problem (3) in which $\hat{B}=B \times_{A} \hat{A}$ and $\operatorname{Ker}(\hat{\varphi})=K$. If $G$ is $\mathcal{G}_{1}$-projective, then (3) splits group theoretically.

Proof. Let $N$ be an open normal subgroup of $G$ as in Lemma 4.1. Consider an open normal subgroup $K$ of $G$ contained in $N$. Decompose $\varphi: \mathbf{G} \rightarrow \mathbf{A}$ into $(\hat{\varphi}: \mathbf{G} \rightarrow \hat{\mathbf{A}}, \bar{\varphi}: \hat{\mathbf{A}} \rightarrow \mathbf{A})$ such that $\operatorname{Ker}(\hat{\varphi})=K$. By Lemma $4.1,(\bar{\varphi}, \alpha)$ is a finite locally solvable embedding problem. Then for each $v \in S$ we may choose a set $\left\{\left(B_{i}, \hat{A}_{i}\right) \mid i \in I_{v}\right\} \subseteq \mathcal{B}_{v} \times \hat{\mathcal{A}}_{v}$ of compatible pairs such that $I_{0}=\{0\}$, the $I_{v}$ are disjoint, and (5) holds. Lemma-Construction 4.2 (a) gives Diagram (4) with $\hat{B}=B \times_{A} \hat{A}$ such that (3) is rigid.

If $G$ is $\mathcal{G}_{1}$-projective, there exists a homomorphism $\gamma: G \rightarrow B$ with $\alpha \circ \gamma=\varphi$. We may assume $N \leq \operatorname{Ker}(\gamma)$, so that $\operatorname{Ker}(\hat{\varphi})=K \leq N \leq \operatorname{Ker}(\gamma)$. Then $\gamma$ induces a homomorphism $\bar{\gamma}: \hat{A} \rightarrow B$ with $\gamma=\bar{\gamma} \circ \hat{\varphi}$. Hence, $\alpha \circ \bar{\gamma} \circ \hat{\varphi}=\alpha \circ \gamma=\varphi=\bar{\varphi} \circ \hat{\varphi}$. Since $\hat{\varphi}$ is surjective, $\alpha \circ \bar{\gamma}=\bar{\varphi}$. The universal property of the cartesian square in (4) gives a homomorphism $\hat{\gamma}: \hat{A} \rightarrow \hat{B}$ such that $\beta \circ \hat{\gamma}=\bar{\gamma}$ and $\hat{\alpha} \circ \hat{\gamma}=\mathrm{id}_{\hat{A}}$. Thus, $\hat{\alpha}$ splits group theoretically.

Notation 4.4. Let $\mathbf{G}=\left(G, \mathcal{G}_{v}\right)_{v \in S}$ be a group pile. For each $v \in S$ and each $G_{v} \in \mathcal{G}_{v}$ let $\bar{G}_{v}$ be the conjugacy class of $G_{v}$ in $G$. Let $\overline{\mathcal{G}}_{v}=\left\{\bar{G}_{v} \mid\right.$ $\left.G_{v} \in \mathcal{G}_{v}\right\}$ be the corresponding topological quotient space. We may identify $\overline{\mathcal{G}}_{v}$ with a set of representatives of the conjugacy classes in $\mathcal{G}_{v}$.

LEMMA 4.5. Let (1) be a finite locally solvable embedding problem for $\mathbf{G}=\left(G, \mathcal{G}_{v}\right)_{v \in S}$. Suppose $\overline{\mathcal{G}}_{v}$ has no isolated points for each $v \in S_{1}$. Then (1) can be dominated by a finite rigid embedding problem (4) with $\hat{B}=$ $B \times_{A} \hat{A}$ such that the following statement holds for each $v \in S_{1}$ : 
(*) For every $B_{v} \in \mathcal{B}_{v}$ there exists $\hat{A}_{v} \in \hat{\mathcal{A}}_{v}$ with $\alpha\left(B_{v}\right)=\bar{\varphi}\left(\hat{A}_{v}\right)$ such that if $\hat{B}^{\prime} \in \hat{\mathcal{B}}_{v}$ and $\hat{\alpha}\left(\hat{B}^{\prime}\right)$ is conjugate to $\hat{A}_{v}$, then $\beta\left(\hat{B}^{\prime}\right)$ is conjugate to $B_{v}$.

Proof. By Lemma 4.1 there is a decomposition $\mathbf{G} \stackrel{\hat{\varphi}}{\longrightarrow} \hat{\mathbf{A}} \stackrel{\bar{\varphi}}{\longrightarrow} \mathbf{A}$ of $\varphi$ where $\hat{\mathbf{A}}=\left(\hat{A}, \hat{\mathcal{A}}_{v}\right)_{v \in S}$ is finite and $(\bar{\varphi}, \alpha)$ is a locally solvable embedding problem.

Let $v \in S_{1}$ and let $n$ be the number of conjugacy classes in $\mathcal{B}_{v}$. For each $B^{\prime} \in \mathcal{B}_{v}$ there is an $\hat{A}^{\prime} \in \hat{\mathcal{A}}_{v}$ compatible with $B^{\prime}$. The set $\left\{G^{\prime} \in\right.$ $\left.\mathcal{G}_{v} \mid \hat{\varphi}\left(G^{\prime}\right)=\hat{A}^{\prime}\right\}$ is non-empty and open-closed in $\mathcal{G}_{v}$. By assumption it contains infinitely many non-conjugate subgroups of $G$. Therefore there is a decomposition $\mathbf{G} \stackrel{\varphi_{1}^{*}}{\longrightarrow} \mathbf{A}^{*} \stackrel{\varphi_{2}^{*}}{\longrightarrow} \hat{\mathbf{A}}$ of $\hat{\varphi}: \mathbf{G} \rightarrow \hat{\mathbf{A}}$ with $\mathbf{A}^{*}=\left(A^{*}, \mathcal{A}_{v}^{*}\right)_{v \in S}$ finite such that there are at least $n$ non-conjugate groups in $\mathcal{A}_{v}^{*}$ mapped by $\varphi_{2}^{*}$ onto $\hat{A}^{\prime}$. Each of them is compatible with $B^{\prime}$, with respect to the embedding problem $\left(\bar{\varphi} \circ \varphi_{2}^{*}, \alpha\right)$. Hence, replacing $\hat{\varphi}: \mathbf{G} \rightarrow \hat{\mathbf{A}}$ by $\varphi_{1}^{*}: \mathbf{G} \rightarrow \hat{\mathbf{A}}^{*}$ and $\bar{\varphi}: \hat{\mathbf{A}} \rightarrow \mathbf{A}$ by $\bar{\varphi} \circ \varphi_{2}^{*}: \mathbf{A}^{*} \rightarrow \mathbf{A}$, we may assume that there are at least $n$ non-conjugate groups in $\hat{\mathcal{A}}$ compatible with $B^{\prime}$. In fact, since $\mathcal{B}_{1}$ is finite, we may assume that the latter statement holds for all $B^{\prime} \in \mathcal{B}_{v}$ and all $v \in S_{1}$.

This allows us to choose for each $v \in S_{1}$ a family of compatible pairs $\left\{\left(B_{i}, \hat{A}_{i}\right) \mid i \in I_{v}\right\} \subseteq \mathcal{B}_{v} \times \hat{\mathcal{A}}_{v}$ such that the set $\left\{B_{i} \mid i \in I_{v}\right\}$ meets all of the $B$-classes of $\mathcal{B}_{v}$ and $\left\{\hat{A}_{i} \mid i \in I_{v}\right\}$ is a system of representatives of the conjugacy classes of $\hat{\mathcal{A}}_{v}$. Indeed, for $v \in S_{1}$ let $\left\{B_{i} \mid i \in J_{v}\right\}$ be a system of representatives of the $B$-classes of $\mathcal{B}_{v}$. By the preceding paragraph, $\left|J_{v}\right| \leq n$, so we may choose for each $i \in J_{v}$ a compatible $\hat{A}_{i} \in \hat{\mathcal{A}}$ such that the $\hat{\hat{A}}_{i}$ 's, $i \in J_{v}$, are non-conjugate. Complete $\left\{\hat{A}_{i} \mid i \in J_{v}\right\}$ to a system $\left\{\hat{A}_{i} \mid i \in I_{v}\right\}$ of representatives of the $\hat{A}$-classes of $\hat{\mathcal{A}}_{v}$ and choose for each $\hat{A}_{i} \in \hat{\mathcal{A}}_{v}$ with $i \in I_{v} \backslash J_{v}$ a compatible $B_{i} \in \mathcal{B}_{v}$. Finally we may change the sets $I_{v}$, if necessary, to assume that they are disjoint and do not contain 0 .

Lemma-Construction Lemma 4.2 gives the required dominating embedding problem (4). Indeed, we choose a compatible pair $\left(B_{0}, \hat{A}_{0}\right) \in \mathcal{B}_{0} \times \hat{\mathcal{A}}_{0}$ and set $\hat{\mathbf{B}}=\left(\hat{B}, \hat{\mathcal{B}}_{v}\right)_{v \in S}$ and $\left\{\hat{B}_{i} \mid i \in I_{v}\right\}$ for each $v \in S$ as in Lemma 4.2. Then (1) is dominated by a finite rigid embedding problem (4).

Now consider $v \in S$. For each $B_{v} \in \mathcal{B}_{v}$ there exist $i \in I_{v}$ and $b \in B$ with $B_{v}=B_{i}^{b}$. Since the pair $\left(B_{i}, \hat{A}_{i}\right)$ is compatible, $\alpha\left(B_{i}\right)=\bar{\varphi}\left(\hat{A}_{i}\right)$. By $(6)$, $\hat{\alpha}\left(\hat{B}_{i}\right)=\hat{\alpha}\left(\hat{\gamma}_{i}\left(\hat{A}_{i}\right)\right)=\hat{A}_{i}$. Choose $\hat{a} \in \hat{A}$ with $\alpha(b)=\bar{\varphi}(\hat{a})$ and set $\hat{A}_{v}=\hat{A}_{i} \hat{a}$. Then $\alpha\left(B_{v}\right)=\bar{\varphi}\left(\hat{A}_{v}\right)$. If for some $\hat{B}^{\prime} \in \hat{\mathcal{B}}_{v}$ the group $\hat{\alpha}\left(\hat{B}^{\prime}\right)$ is conjugate to $\hat{A}_{v}$, then $\hat{\alpha}\left(\hat{B}^{\prime}\right)$ is conjugate to $\hat{A}_{i}$. Therefore, by Lemma $4.2(\mathrm{~b}), \beta\left(\hat{B}^{\prime}\right)$ is conjugate to $B_{i}$, hence to $B_{v}$, as is required by $(*)$. 
Remark 4.6. In Lemma 4.5 let (4) satisfy statement (*). Let

$$
\left(\varphi^{*}: \mathbf{G} \rightarrow \mathbf{A}^{*}, \alpha^{*}: \mathbf{B}^{*} \rightarrow \mathbf{A}^{*}\right)
$$

be another finite locally solvable embedding problem which dominates (3) such that $B^{*}=\hat{B} \times{ }_{\hat{A}} A^{*}$. Then (7) dominates (1) and satisfies statement (*) of Lemma 4.5. This allows us to replace (3) by an embedding problem with additional properties.

\section{$\S 5$. Free products}

We follow Melnikov-Haran and define a free product of profinite groups indexed by a profinite space. A special free product will be shown to have all necessary properties entering in the definition of a Cantor group pile in the next section.

The basic notion underlying the free product of profinite groups is that of a sheaf of profinite groups [Mel, (1.13)]. It is a triple $(X, \tau, T)$ of profinite spaces $X, T$ with a surjective continuous map $\tau: X \rightarrow T$ such that $X_{T, t}=\tau^{-1}(t)$ is a profinite group for each $t \in T$ and the map $(x, y) \mapsto x^{-1} y$ from $\{(x, y) \in X \times X \mid \tau(x)=\tau(y)\}$ into $X$ is continuous.

The simplest sheaves of profinite groups are the constant sheaves [Mel, (1.13)]: Let $\Gamma$ be a profinite group and let $T$ be a profinite space. Consider the triple $(\Gamma \times T$, pr, $T)$, where pr: $\Gamma \times T \rightarrow T$ is the projection on the second coordinate. For each $t \in T$ the fiber $\operatorname{pr}^{-1}(t)=\Gamma \times\{t\}$ is a profinite group isomorphic to $\Gamma$ by $(\gamma, t) \mapsto \gamma$.

DATA 5.1. We retain the finite set $S$ from Section 3 and its partition $S=S_{0} \cup S_{1}$ with $S_{0}=\{0\}$ and $1 \notin S$. For each $v \in S$ let $\Gamma_{v}$ be a finitely generated profinite group and $T_{v}$ a profinite space such that $T_{0}=\{0\}$. Suppose $\Gamma_{0}$ is profinite free, $\Gamma_{v}$ is nontrivial for each $v \in S_{1}$, and the $T_{v}$ 's, $v \in S$, are disjoint. Thus, $T_{1}=\bigcup_{v \in S_{1}} T_{v}$ is a profinite space and $T=$ $T_{0} \cup T_{1}=\bigcup_{v \in S} T_{v}$ are partitions of $T$ into open-closed subsets.

We combine the constant sheaves $\left(\Gamma_{v} \times T_{v}, \mathrm{pr}, T_{v}\right)$ to a semi-constant sheaf: Set $X_{T}=\bigcup_{v \in S}\left(\Gamma_{v} \times T_{v}\right)$ and let pr: $X_{T} \rightarrow T$ be the unique map which extends the projection maps pr: $\Gamma_{v} \times T_{v} \rightarrow T_{v}$. Then $\mathbf{X}_{T}=$ $\left(X_{T}, \mathrm{pr}, T\right)$ is a sheaf of profinite groups with $X_{T, t}=\Gamma_{v} \times\{t\}$ for $v \in S$ and $t \in T_{v}$.

Let $G_{T}=\mathbb{F}_{T} \mathbf{X}_{T}$ be the free product of $\mathbf{X}_{T}$ [Mel, (1.14)]. Thus, $G_{T}$ is a profinite group together with a continuous map $\omega: X_{T} \rightarrow G_{T}$ with the following properties: 
(1a) The restriction of $\omega$ to each fiber $X_{T, t}$ is an injective homomorphism $X_{T, t} \rightarrow G_{T}$ [Mel, (1.15)].

(1b) Given a profinite group $B$ and a continuous map $\beta: X_{T} \rightarrow B$, whose restriction to each fiber $X_{T, t}$ is a homomorphism, there is a unique homomorphism $\gamma: G_{T} \rightarrow B$ such that $\gamma \circ \omega=\beta$.

For each $t \in T$ put $G_{T, t}=\omega\left(X_{T, t}\right)$. Then $G_{T, t} \cong \Gamma_{v}$ for each $t \in T_{v}$ and each $v \in S$. By [Mel, (1.16), (1.17)], $G_{T}=\mathbb{*}_{t \in T} G_{T, t}$ is the (inner) free product of the groups $G_{T, t}, t \in T$. This means that the map $\bar{\omega}: T \rightarrow \operatorname{Subgr}\left(G_{T}\right)$ defined by $\bar{\omega}(t)=G_{T, t}$ is étale continuous and every continuous map from $\bigcup_{t \in T} G_{T, t}$ into a profinite group $H$, whose restriction to each $G_{T, t}$ is a homomorphism $G_{T, t} \rightarrow H$, admits a unique extension to a homomorphism $G_{T} \rightarrow H$. By [Mel, (4.9)],

(2) $G_{T, t}$ and $G_{T, t^{\prime}}$ are non-conjugate if $t, t^{\prime} \in T$ are distinct.

The partition $T=\bigcup_{v \in S} T_{v}$ into open-closed sets yields a free decomposition $G_{T}=\Re_{v \in S} \circledast_{t \in T} G_{T, t}[\mathrm{Mel},(1.7)]$. Since $T_{0}=\{0\}$, we get

$$
G_{T}=G_{T, 0} * \prod_{v \in S_{1}} \prod_{t \in T_{v}} G_{T, t} .
$$

For each $v \in S$ let $\mathcal{G}_{T, v}^{\prime}=\left\{G_{T, t} \mid t \in T_{v}\right\}, \mathcal{G}_{T, v}=\left\{G_{T, t}^{g} \mid t \in T_{v}, g \in\right.$ $\left.G_{T}\right\}, \mathcal{G}_{T, 1}=\bigcup_{v \in S_{1}} \mathcal{G}_{T, v}$, and $\mathcal{G}_{T}=\bigcup_{v \in S} \mathcal{G}_{T, v}$.

The following result of Efrat is an analog of a lemma of Gaschütz [FrJ, Lemma 17.7.2]. We will have two opportunities to use this result.

Lemma 5.2. ([Efr, Main Theorem]) Let $\alpha: B \rightarrow A$ be an epimorphism of finite groups, $A_{1}, \ldots, A_{n}$ subgroups of $A$, and $B_{1}, \ldots, B_{n}$ subgroups of B. Suppose $A=\left\langle A_{1}, \ldots, A_{n}\right\rangle, B=\left\langle B_{1}, \ldots, B_{n}\right\rangle$, and $\alpha\left(B_{i}\right)$ is a conjugate of $A_{i}$ for $i=1, \ldots, n$. Then there exist $b_{1}, \ldots, b_{n} \in B$ such that $B=$ $\left\langle B_{1}^{b_{1}}, \ldots, B_{n}^{b_{n}}\right\rangle$ and $\alpha\left(B_{i}^{b_{i}}\right)=A_{i}$ for $i=1, \ldots, n$.

Proposition 5.3. The structure $\mathbf{G}_{T}=\left(G_{T}, \mathcal{G}_{T, v}\right)_{v \in S}$ is a group pile. It satisfies:

(a) The map $\bar{\omega}: T \rightarrow \operatorname{Subgr}\left(G_{T}\right)$ given by $t \mapsto G_{T, t}$ is strictly continuous (and not only étale continuous).

(b) The map $T \times G_{T} \rightarrow \operatorname{Subgr}\left(G_{T}\right)$ given by $(t, g) \mapsto G_{T, t}^{g}$ is strictly continuous. 
(c) $G_{T}=\left\langle G_{T, 0}, \mathcal{G}_{T, v}^{\prime} \mid v \in S_{1}\right\rangle$; in particular $\mathbf{G}_{T}$ is self-generated.

(d) Suppose $T=\varliminf_{j \in J} T^{(j)}$ and $T^{(j)}=\bigcup_{v \in S} T_{v}^{(j)}$, where each $T_{v}^{(j)}$ is open-closed in $T^{(j)}, T_{0}^{(j)}=\{0\}$, and the maps $T \rightarrow T^{(j)}$ map $T_{v}$ into $T_{v}^{(j)}$ for all $v \in S$ and $j \in J$. Then $G_{T}=\lim _{j \in J} G_{T^{(j)}}$. Moreover, for each $j \in J$, if $t \in T$ and $t^{(j)}$ is its image in $T^{(j)}$, then the induced map $G_{T, t} \rightarrow G_{T^{(j)}, t^{(j)}}$ is an isomorphism.

(e) If $T$ has a countable basis for its topology, then $G_{T}$ is countably generated.

(f) $\mathcal{G}_{T}=\bigcup_{v \in S} \mathcal{G}_{T, v}$, is a partition into open-closed subsets of $\mathcal{G}_{T}$; moreover, for every $v \in S$ and $H \in \mathcal{G}_{T, v}$ we have $H \cong \Gamma_{v}$.

(g) For each $v \in S_{1}$, the set $\mathcal{G}_{T, v}^{\prime}$ is a closed system of representatives of the $G_{T}$-classes of $\mathcal{G}_{T, v}$ and the space $\overline{\mathcal{G}}_{T, v}$ of the $G_{T}$-classes of the groups in $\mathcal{G}_{T, v}$ is homeomorphic to $T_{v}$.

(h) If $T_{1}$ has no isolated points, then every finite self-generated locally solvable embedding problem for $\mathbf{G}_{T}$ is solvable.

(i) $G_{T}$ is $\mathcal{G}_{T, 1}$-projective.

Proof. First we note that for each $v \in S$ the subset $\mathcal{G}_{T, v}$ of $\operatorname{Subgr}\left(G_{T}\right)$ is closed. Indeed, by (b) proven below, $(t, g) \mapsto G_{T, t}^{g}$ is a continuous map of profinite spaces $T_{v} \times G_{T} \rightarrow \operatorname{Subgr}\left(G_{T}\right)$. As such it is a closed map, hence its image $\mathcal{G}_{T, v}$ is closed.

Proof of (a). Let $t \in T$, say, $t \in T_{v} \subseteq T$ with $v \in S$, and let $N$ be an open normal subgroup of $G_{T}$. The composed map $\omega_{N}: X_{T} \rightarrow G_{T} / N$ of $\omega: X_{T} \rightarrow G_{T}$ with the quotient map $G_{T} \rightarrow G_{T} / N$ is continuous. Since $G_{T} / N$ is discrete, for each $(\gamma, t) \in X_{T, t}=\Gamma_{v} \times\{t\}$ there are open-closed neighborhoods $U$ of $\gamma$ in $\Gamma_{v}$ and $V$ of $t$ in $T_{v} \subseteq T$ such that $\omega_{N}(U \times V)=$ $\left\{\omega_{N}(\gamma, t)\right\}$. By the compactness of $X_{t}$ we may assume that $V$ does not depend on $\gamma$. Then, for all $t^{\prime} \in V$, we have $\omega_{N}\left(X_{T, t^{\prime}}\right)=\omega_{N}\left(X_{T, t}\right)$. By definition, $\bar{\omega}(t)=\omega\left(X_{T, t}\right)$. Hence, $\bar{\omega}\left(t^{\prime}\right) N=\bar{\omega}(t) N$. Consequently, $\bar{w}: T \rightarrow$ $\operatorname{Subgr}\left(G_{T}\right)$ is continuous.

Proof of (b). This follows from (a).

Proof of (c). See [Mel, Lemma 1.15].

Proof of $(\mathrm{d})$. The sheaf $\mathbf{X}_{T}=\left(X_{T}, \mathrm{pr}, T\right)$ is the inverse limit of the sheaves $\mathbf{X}_{T^{(j)}}=\left(X_{T^{(j)}}\right.$, pr, $\left.T^{(j)}\right)$, where the maps $X_{T} \rightarrow X_{T^{(j)}}$ are induced from the maps $\Gamma_{v} \times T_{v} \rightarrow \Gamma_{v} \times T_{v}^{(j)}$; therefore they map the fibers 
of $X_{T} \rightarrow T$ isomorphically onto the fibers of $X_{T^{(j)}} \rightarrow T^{(j)}$. We have $G_{T^{(j)}}=\Re_{T^{(j)}} \mathbf{X}_{T^{(j)}}$. Then $G_{T}=\lim _{i} G_{T^{(j)}}$ [Mel, (2.4)].

As for the last assertion, we have $t \in T_{v}$ for some $v \in S$. Then $t^{(j)} \in$ $T_{v}^{(j)}$. Both $X_{T, t}$ and $X_{T^{(j)}, t^{(j)}}$ are isomorphic copies of $\Gamma_{v}$ (by (1a)) and the map $G_{T, t} \rightarrow G_{T^{(j)}, t^{(j)}}$ is induced by the identity map of $\Gamma_{v}$. Hence it is an isomorphism.

Proof of (e). If $T$ is finite, then by (c), $G_{T}$ is generated by finitely many finitely generated profinite groups, so it is finitely generated.

In the general case we may write $T$ as the inverse limit of a sequence of finite sets $T^{(j)}$ as in (d). By (d) and the preceding paragraph, $G_{T}$ is the inverse limit of a sequence of finitely generated profinite groups, hence is countably generated.

Proof of (f). Since a continuous map of profinite spaces is closed, by (b), the sets $\mathcal{G}$ and $\mathcal{G}_{T, v}$ are closed in $\operatorname{Subgr}\left(G_{T}\right)$. By [Mel, (1.17)], $G_{T}$ is the free product of the groups $\left\{G_{T, t}\right\}_{t \in T}$. Hence, by $(2)$, the sets $\mathcal{G}_{T, v}$ are disjoint. Therefore, the sets $\mathcal{G}_{T, v}$ are open in $\mathcal{G}_{T}$ as well.

Let $t \in T_{v}$ and $g \in G_{T}$. Then $X_{T, t} \cong \Gamma_{v}$. By (1a), $G_{T, t} \cong X_{T, t}$. Hence $G_{T, t}^{g} \cong G_{T, t} \cong X_{T, t} \cong \Gamma_{v}$.

Proof of (g). The map $\bar{\omega}$ defined in (a) maps the profinite space $T_{v}$ continuously onto the closed subset $\mathcal{G}_{T, v}^{\prime}$ of $\mathcal{G}_{T, v}$. By $(2), \bar{\omega}$ is injective, so it is a homeomorphism. Moreover, by $(2), \mathcal{G}_{T, v}^{\prime}$ meets exactly once each conjugacy class in $\mathcal{G}_{T, v}$. Hence, $\mathcal{G}_{T, v}^{\prime}$ is a closed set of representatives of the conjugacy classes of $\mathcal{G}_{T, v}$. Since $\mathcal{G}_{T, v}^{\prime}$ is homeomorphic to $\overline{\mathcal{G}}_{T, v}$, it is also homomorphic to $T_{v}$.

Proof of (h). Let

$$
\left(\varphi: \mathbf{G}_{T} \rightarrow \mathbf{A}, \alpha: \mathbf{B} \rightarrow \mathbf{A}\right)
$$

be a finite self-generated locally solvable embedding problem with $\mathbf{A}=$ $\left(A, \mathcal{A}_{v}\right)_{v \in S}$ and $\mathbf{B}=\left(B, \mathcal{B}_{v}\right)_{v \in S}$. We break the proof that (4) is solvable into several parts.

PART A: Making (4) rigid. By (c), $G_{T}=\left\langle G_{T, 0}, \mathcal{G}_{T, v}\right\rangle_{v \in S_{1}}$. Hence, $A=\left\langle A_{0}, \mathcal{A}_{v}\right\rangle_{v \in S_{1}}$, where $A_{0}=\varphi\left(G_{T, 0}\right)$. Since $\mathcal{B}$ is self-generated, there exists $B_{0}^{*} \in \mathcal{B}_{0}$ such that $B=\left\langle B_{0}^{*}, \mathcal{B}_{v}\right\rangle_{v \in S_{1}}$. Next note that $\alpha\left(B_{0}^{*}\right)$ belongs to $\mathcal{A}_{0}$, so $\alpha\left(B_{0}^{*}\right)$ is conjugate in $A$ to $A_{0}$. Also, $\alpha\left(\mathcal{B}_{v}\right)=\mathcal{A}_{v}$ for each $v \in S_{1}$. Hence, by Lemma 5.2 , there exists $B_{0} \in \mathcal{B}_{0}$ such that $B=\left\langle B_{0}, \mathcal{B}_{v}\right\rangle_{v \in S_{1}}$ 
and $\alpha\left(B_{0}\right)=A_{0}$. Since (4) is locally solvable, there exist a $g \in G_{T}$ and an epimorphism $\varepsilon_{0}: G_{T, 0}^{g} \rightarrow B_{0}$ such that $\alpha \circ \varepsilon_{0}=\left.\varphi\right|_{G_{T, 0}^{g}}$. In particular, $\operatorname{rank}\left(G_{T, 0}\right)=\operatorname{rank}\left(G_{T, 0}^{g}\right) \geq \operatorname{rank}\left(B_{0}\right)$. Since $G_{T, 0} \cong \Gamma_{0}$ and $\Gamma_{0}$ is free, we may apply Gaschütz' lemma [FrJ, 17.7.2], to find an epimorphism $\delta_{0}: G_{T, 0} \rightarrow B_{0}$ such that $\alpha \circ \delta_{0}=\left.\varphi\right|_{G_{T, 0}}$. By Lemma 4.1, there is a decomposition $\mathbf{G}_{T} \stackrel{\hat{\varphi}}{\longrightarrow} \hat{A} \stackrel{\bar{\varphi}}{\longrightarrow} \mathbf{A}$ such that $\operatorname{Ker}(\hat{\varphi}) \leq \operatorname{Ker}\left(\delta_{0}\right)$ and $(\bar{\varphi}: \hat{\mathbf{A}} \rightarrow \mathbf{A}, \alpha: \mathbf{B} \rightarrow \mathbf{A})$ is a finite locally solvable embedding problem. In particular, with $\hat{A}_{0}=\hat{\varphi}\left(G_{T, 0}\right)$, the map $\delta_{0}$ defines an epimorphism $\gamma_{0}: \hat{A}_{0} \rightarrow B_{0}$ such that $\alpha \circ \gamma_{0}=\left.\bar{\varphi}\right|_{\hat{A}_{0}}$. Thus, $\left(B_{0}, \hat{A}_{0}\right)$ is a compatible pair.

For each $v \in S_{1}$ we choose a finite set $I_{v}$ and compatible pairs $\left(B_{i}, \hat{A}_{i}\right) \in$ $\mathcal{B}_{v} \times \hat{A}_{v}, i \in I_{v}$, such that Condition (5) of Lemma 4.2 is satisfied, the $I_{v}$ 's are disjoint, and $I_{0}=\{0\}$. By that lemma, there is a commutative diagram

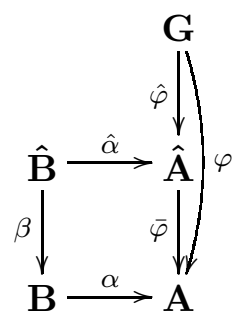

of group piles such that $\hat{\mathbf{B}}$ is finite, $\hat{\alpha}$ is a rigid epimorphism, and $\beta$ is an epimorphism.

Unfortunately, $\hat{\mathbf{B}}$ need not be self-generated. Nevertheless, by Lemma 4.2 , there exists a homomorphism $\hat{\gamma}_{0}: \hat{A}_{0} \rightarrow \hat{B}$ such that $\beta \circ \hat{\gamma}_{0}=\gamma_{0}$ and $\hat{\alpha} \circ \hat{\gamma}_{0}=\operatorname{id}_{\hat{A}_{0}}$. Let $B_{0}^{\prime}=\hat{\gamma}_{0}\left(\hat{A}_{0}\right), B^{\prime}=\left\langle B_{0}^{\prime}, \hat{\mathcal{B}}_{v}\right\rangle_{v \in S_{1}}, \mathcal{B}_{0}^{\prime}=\left\{\left(B_{0}^{\prime}\right)^{b^{\prime}} \mid b^{\prime} \in B^{\prime}\right\}$, $\mathcal{B}_{v}^{\prime}=\hat{\mathcal{B}}_{v}$ for each $v \in S_{1}, \mathbf{B}^{\prime}=\left(B^{\prime}, \mathcal{B}_{v}^{\prime}\right)_{v \in S}, \beta^{\prime}=\left.\beta\right|_{B^{\prime}}$, and $\alpha^{\prime}=\left.\hat{\alpha}\right|_{B^{\prime}}$. Then $\mathbf{B}^{\prime}$ is a self-generated finite group pile. Moreover, since $B=\left\langle B_{0}, \mathcal{B}_{v}\right\rangle_{v \in S_{1}}$, and $\beta^{\prime}\left(B_{0}^{\prime}\right)=\beta^{\prime}\left(\hat{\gamma}_{0}\left(\hat{A}_{0}\right)\right)=\gamma_{0}\left(\hat{A}_{0}\right)=B_{0}$, the morphism $\beta^{\prime}: \mathbf{B}^{\prime} \rightarrow \mathbf{B}$ is an epimorphism. Similarly, $\alpha^{\prime}: \mathbf{B}^{\prime} \rightarrow \hat{A}$ is an epimorphism. Moreover, $\alpha^{\prime}$ is rigid, because $\hat{\alpha}$ is rigid. In particular,

$$
\left(\hat{\varphi}: \mathbf{G}_{T} \rightarrow \hat{\mathbf{A}}, \alpha^{\prime}: \mathbf{B}^{\prime} \rightarrow \hat{\mathbf{A}}\right)
$$

is locally solvable. Every solution of (6) yields a solution of (4). Consequently, we may assume without loss that (4) is rigid.

PART B: Selection of subgroups. For each $v \in S_{1}$ consider again the closed subset $\mathcal{G}_{T, v}^{\prime}$ of $\mathcal{G}_{T, v}$ and the subset $\mathcal{A}_{v}^{\prime}=\varphi\left(\mathcal{G}_{T, v}^{\prime}\right)$ of $\mathcal{A}_{v}$. Since 
$\mathcal{G}_{T, v}=\left(\mathcal{G}_{T, v}^{\prime}\right)^{G}$, we have $\mathcal{A}_{v}=\left(\mathcal{A}_{v}^{\prime}\right)^{A}$. Moreover, with $A_{0}=\varphi\left(G_{0}\right)$ we get, by (c), that $A=\left\langle A_{0}, \mathcal{A}_{v}^{\prime} \mid v \in S_{1}\right\rangle$. Next we choose $B_{0} \in \mathcal{B}_{0}$ and subsets $\mathcal{B}_{v}^{\prime} \subseteq \mathcal{B}_{v}, v \in S_{1}$, such that

(7) $B=\left\langle B_{0}, \mathcal{B}_{v}^{\prime} \mid v \in S_{1}\right\rangle$ and $\mathcal{B}_{v}^{\prime}$ meets every $B$-class in $\mathcal{B}_{v}$ for each $v \in S_{1}$

(e.g. $\left.\mathcal{B}_{v}^{\prime}=\mathcal{B}_{v}\right)$. Then $\alpha\left(\mathcal{B}_{v}^{\prime}\right) \subseteq \alpha\left(\mathcal{B}_{v}\right)=\mathcal{A}_{v}=\left(\mathcal{A}_{v}^{\prime}\right)^{A}$. Therefore, we may find disjoint finite sets $J_{v}, v \in S_{1}$, not containing 0 and for each $v \in S_{1}$ label the elements of $\mathcal{A}_{v}^{\prime}$ as $A_{v, j}$ and the elements of $\mathcal{B}_{v}^{\prime}$ as $B_{v, j}, j \in J_{v}$, where the $A_{j}$ 's need not be distinct, the $B_{j}$ 's need not be distinct, but $\alpha\left(B_{v, j}\right)$ is a conjugate of $A_{v, j}$ in $A, j \in J_{v}$. In addition, we put $J_{0}=\{0\}, B_{0,0}=B_{0}$, $A_{0,0}=A_{0}$, and note that $\alpha\left(B_{0,0}\right)$ is conjugate to $A_{0,0}$. By (7) and by Lemma 5.2 , we may replace the $B_{j, v}$ 's by appropriate conjugate subgroups in $B$ such that after the replacement

$\left(7^{\prime}\right) B=\left\langle B_{0,0}, \mathcal{B}_{v}^{\prime} \mid v \in S_{1}\right\rangle, B_{0,0} \in \mathcal{B}_{0}, \mathcal{B}_{v}^{\prime}$ meets every conjugacy class in $\mathcal{B}_{v}$, and $\alpha\left(B_{v, j}\right)=A_{v, j}$ for all $j \in J_{v}$ and $v \in S$.

PART C: Partition of $T$. By (a), for each $v \in S_{1}$, the map $t \mapsto \varphi\left(G_{T, t}\right)$ from $T_{v}$ to $\mathcal{A}_{v}$ is continuous. Hence, each of the subsets $T_{v, j}=\left\{t \in T_{v} \mid\right.$ $\left.\varphi\left(G_{T, t}\right)=A_{v, j}\right\}$ of $T_{v}$ is open-closed. Moreover, $T_{v, j} \neq \emptyset$, because $\varphi: \mathbf{G}_{T} \rightarrow$ A is surjective. However, $T_{v, j}=T_{v, j^{\prime}}$ for distinct $j, j^{\prime} \in J_{v}$ if $A_{v, j}=A_{v, j^{\prime}}$. Nevertheless, since $T_{v}$ has no isolated points, we may partition each $T_{v, j}$ such that they become disjoint and get a partition $T_{v}=\bigcup_{j \in J_{v}} T_{v, j}$ into open-closed subsets such that $\varphi\left(G_{T, t}\right)=A_{v, j}$ for each $t \in T_{v, j}$ and each $j \in J_{v}$. In addition, we set $T_{0,0}=T_{0}=\{0\}$. Then $\varphi\left(G_{T, t}\right)=A_{v, j}$ for $t \in T_{v, j}, j \in J_{v}$, and $v \in S$. Let $\alpha_{v, j}^{\prime}$ be the inverse of the isomorphism $\alpha: B_{v, j} \rightarrow A_{v, j}$.

PART D: Solution of (4). For each $v \in S$ and $j \in J_{v}$ let $X_{v, j}=$ $\operatorname{pr}^{-1}\left(T_{v, j}\right)$. Then, $X_{T}=\bigcup_{v \in S} \bigcup_{j \in J_{v}} X_{v, j}$ is a partition of $X$ into openclosed subsets. If $x \in X_{v, j}$, then $t=\operatorname{pr}(x) \in T_{v, j}, \omega(x) \in G_{T, t}$, and $\varphi(\omega(x)) \in A_{v, j}$, so that $\alpha_{v, j}^{\prime}(\varphi(\omega(x)))$ is well defined. We may therefore define a map $\beta: X_{T} \rightarrow B$ by $\left.\beta\right|_{X_{v, j}}=\left.\alpha_{v, j}^{\prime} \circ \varphi \circ \omega\right|_{X_{v, j}}$. It satisfies

$$
\left.\alpha \circ \beta\right|_{X_{v, j}}=\left.\alpha \circ \alpha_{v, j}^{\prime} \circ \varphi \circ \omega\right|_{X_{v, j}}=\left.\varphi \circ \omega\right|_{X_{v, j}}
$$

for $v \in S$ and $j \in J_{v}$. By (1b), $\beta$ defines a homomorphism $\gamma: G_{T} \rightarrow B$ such that $\gamma \circ \omega=\beta$. By (8), $(\alpha \circ \gamma) \circ \omega=\alpha \circ \beta=\varphi \circ \omega$. Therefore, by the uniqueness property (1b), $\alpha \circ \gamma=\varphi$. Further, for each $t \in T_{v, j}$ we have 
$X_{T, t} \subseteq X_{v, j}$, so $\gamma\left(G_{T, t}\right)=\gamma\left(\omega\left(X_{T, t}\right)\right)=\beta\left(X_{T, t}\right)=\alpha_{v, j}^{\prime} \circ \varphi \circ \omega\left(X_{T, t}\right)=$ $\alpha_{v, j}^{\prime} \circ \varphi\left(G_{T, t}\right)=\alpha_{v, j}^{\prime}\left(A_{v, j}\right)=B_{v, j}$. It follows from $\left(7^{\prime}\right)$ that $\gamma(G)=B$. In addition, $\gamma\left(\mathcal{G}_{T, 0}\right)=\mathcal{B}_{0}$ and $\gamma\left(\mathcal{G}_{T, v}\right)=\gamma\left(\left(\mathcal{G}_{T, v}^{\prime}\right)^{G}\right)=\left(\mathcal{B}_{v}^{\prime}\right)^{B}=\mathcal{B}_{v}$ for each $v \in S_{1}$. Consequently, $\gamma: \mathbf{G} \rightarrow \mathbf{B}$ is an epimorphism solving embedding problem (4).

Proof of (i). Let $\alpha: B \rightarrow A$ be an epimorphism of finite groups and $\varphi: G_{T} \rightarrow A$ be an epimorphism. Suppose for each $H \in \mathcal{G}_{T, 1}$ there exists a homomorphism $\gamma_{H}: H \rightarrow B$ such that $\alpha \circ \gamma_{H}=\left.\varphi\right|_{H}$. We have to produce a homomorphism $\gamma: G_{T} \rightarrow B$ such that $\alpha \circ \gamma=\varphi$.

To that end we write $T$ as the inverse limit of finite spaces $\lim _{j \in J} T^{(j)}$. By (d), $\varphi$ factors through $G_{T^{(j)}}$ for some $j \in J$. Moreover, the map $G_{T} \rightarrow$ $G_{T^{(j)}}$ is injective on each $H \in \mathcal{G}_{T}$. By assumption, $G_{T, 0}$ is isomorphic to the free finitely generated profinite group $\Gamma_{0}$. Hence, $G_{T^{(j)}, 0}$ is a free finitely generated profinite group and each $H^{(j)} \in \mathcal{G}_{T^{(j)}, 1}$ satisfies the condition of local solvability. We may therefore assume that $T$ is finite and $G_{T}=G_{T, 0}$ * $*_{t \in T_{1}} G_{T, t}$ is the free product of finitely many profinite groups, with $G_{T, 0}$ free. By [FrJ, Cor. 22.4.5], $G_{T, 0}$ is projective, so there exists $\gamma_{0}: G_{T, 0} \rightarrow B$ such that $\alpha \circ \gamma_{0}=\left.\varphi\right|_{G_{T, 0}}$. By assumption, for each $t \in T_{1}$ there is a group $B_{t} \in \mathcal{B}$ and an epimorphism $\gamma_{t}: G_{T, t} \rightarrow B_{t}$ such that $\alpha \circ \gamma_{t}=\left.\varphi\right|_{G_{T, t}}$. These maps extend to a homomorphism $\gamma: G_{T} \rightarrow B$ such that $\alpha \circ \gamma=\varphi$, as claimed.

\section{§6. Iwasawa criterion for group piles}

Iwasawa has characterized the free profinite group $\hat{F}_{\omega}$ of countable rank as a profinite group of countable rank for which every finite embedding problem is solvable. Using the language of piles and the same method of proof, we characterize the free product of groups of finitely many isomorphism types over Cantor sets by essentially the same condition, namely solvability of finite embedding problems, more precisely by Condition (1) below.

Let $\left(S, S_{0}, S_{1}, \Gamma_{v}, T_{v}\right)_{v \in S}$ be a data as in Data 5.1.

Definition 6.1. Let $\mathbf{G}=\left(G, \mathcal{G}_{v}\right)_{v \in S}$ be a group pile. We say that $\mathbf{G}$ is a Cantor group pile over $\left(\Gamma_{v}\right)_{v \in S}$ if it satisfies the following conditions:

(1a) $\operatorname{rank}(G) \leq \aleph_{0}$.

(1b) For each $v \in S_{1}$, the space $\overline{\mathcal{G}}_{v}$ of the $G$-classes of $\mathcal{G}_{v}$ has no isolated points. 
(1c) $\mathcal{G}=\bigcup_{v \in S} \mathcal{G}_{v}$, where, for each $v \in S, \mathcal{G}_{v}$ is an open-closed subset of $\mathcal{G}$ and $H \cong \Gamma_{v}$ for every $H \in \mathcal{G}_{v}$.

(1d) $\mathbf{G}$ is self-generated and every finite locally solvable self-generated embedding problem for $\mathbf{G}$ is solvable.

The name "Cantor group pile" is justified by Conditions (1a) and (1b). By [HaJ1, Lemma 1.2], they are equivalent to the spaces $\overline{\mathcal{G}}_{v}, v \in S_{1}$, being homeomorphic to the Cantor middle third set, which we refer to as the Cantor space. Thus, the following result is a special case of Proposition 5.3.

Corollary 6.2. For each $v \in S_{1}$ let $T_{v}$ be a homeomorphic copy of the Cantor space. Then $\mathbf{G}_{T}=\left(G_{T}, \mathcal{G}_{T, v}\right)_{v \in S}$ is a Cantor group pile over $\left(\Gamma_{v}\right)_{v \in S}$.

Having constructed a Cantor group pile over $\left(\Gamma_{v}\right)_{v \in S}$ we now prove its uniqueness. The proof is modeled after the proof of [FrJ, Lemma 24.4.7].

Proposition 6.3. Let $\mathbf{G}=\left(G, \mathcal{G}_{v}\right)_{v \in S}$ and $\mathbf{G}^{\prime}=\left(G^{\prime}, \mathcal{G}_{v}^{\prime}\right)_{v \in S}$ be Cantor group piles over $\left(\Gamma_{v}\right)_{v \in S}$. Then $\mathbf{G} \cong \mathbf{G}^{\prime}$.

Proof. Choose descending sequences $G=K_{1} \geq K_{2} \geq \cdots$ and $G^{\prime}=$ $K_{1}^{\prime} \geq K_{2}^{\prime} \geq \cdots$ of open normal subgroups of $G$ and $G^{\prime}$, respectively, with $\bigcap_{n=1}^{\infty} K_{n}=1$ and $\bigcap_{n=1}^{\infty} K_{n}^{\prime}=1$.

Inductively define descending sequences $L_{1} \geq L_{2} \geq \cdots$ and $L_{1}^{\prime} \geq L_{2}^{\prime} \geq$ .. of open normal subgroups of $G$ and $G^{\prime}$, respectively, and isomorphisms $\theta_{n}: \mathbf{G} / L_{n} \rightarrow \mathbf{G}^{\prime} / L_{n}^{\prime}$, for $n=1,2, \ldots$ satisfying the following conditions, for every $n \geq 1$ :

(3a) $L_{n} \leq K_{n}$.

(3b) $L_{n}^{\prime} \leq K_{n}^{\prime}$.

(3c) If $n \geq 2$, then the following diagram, in which the horizontal arrows are the quotient maps (and hence $\lambda_{n} \circ \pi_{n}=\pi_{n-1}$ and $\lambda_{n}^{\prime} \circ \pi_{n}^{\prime}=\pi_{n-1}^{\prime}$ ), is commutative:

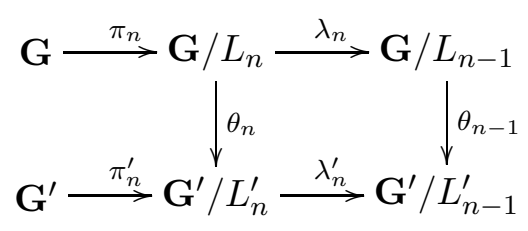


(3d) The following condition holds for each $v \in S$ : for each $H \in \mathcal{G}_{v}$ there exists $H^{\prime} \in \mathcal{G}_{v}^{\prime}$ and for each $H^{\prime} \in \mathcal{G}_{v}^{\prime}$ there exists $H \in \mathcal{G}_{v}$ with an isomorphism $\gamma_{0}: H \rightarrow H^{\prime}$ such that $\left.\theta_{n} \circ \pi_{n}\right|_{H}=\pi_{n}^{\prime} \circ \gamma_{0}$.

Then $\mathbf{G}=\lim _{n} \mathbf{G} / L_{n}$ and $\mathbf{G}^{\prime}=\lim _{n} \mathbf{G}^{\prime} / L_{n}^{\prime}$, and the isomorphisms $\theta_{1}, \theta_{2}, \theta_{3}, \ldots$ define an isomorphism $\theta: \overleftarrow{\mathbf{G}} \rightarrow_{\rightarrow}^{n} \mathbf{G}^{\prime}$. (This follows already from (3a) $-(3 \mathrm{c})$; we need (3d) only for the induction step.)

For $n=1$ let $L_{1}=G, L_{1}^{\prime}=G^{\prime}$, and set $\theta_{1}$ to be the trivial map. Then (3a) and (3b) hold trivially, (3c) is vacuous, and (3d) holds by (1c).

Now let $n \geq 2$ and suppose (3) holds for $n-1$. In particular, $L_{n-1}$, $L_{n-1}^{\prime}$, and the isomorphism $\theta_{n-1}: G / L_{n-1} \rightarrow G^{\prime} / L_{n-1}^{\prime}$ have already been constructed and

$\left(3^{\prime} \mathrm{d}\right)$ the following condition holds for each $v \in S$ : for each $H \in \mathcal{G}_{v}$ there exists $H^{\prime} \in \mathcal{G}_{v}^{\prime}$ and for each $H^{\prime} \in \mathcal{G}_{v}^{\prime}$ there exists $H \in \mathcal{G}_{v}$ with an isomorphism $\gamma_{0}: H \rightarrow H^{\prime}$ such that $\left.\theta_{n-1} \circ \pi_{n-1}\right|_{H}=\pi_{n-1}^{\prime} \circ \gamma_{0}$.

Choose an open normal subgroup $L_{n}^{\prime}$ of $G^{\prime}$ such that $L_{n}^{\prime} \leq K_{n}^{\prime} \cap L_{n-1}^{\prime}$. This gives $(3 \mathrm{~b})$. Let $m=\left(G^{\prime}: L_{n}^{\prime}\right)$. Then, for each $H^{\prime} \in \mathcal{G}^{\prime}$, we have $\left(H^{\prime}: H^{\prime} \cap L_{n}^{\prime}\right)=\left(H^{\prime} L_{n}^{\prime}: L_{n}^{\prime}\right) \leq m$, so, in the notation of Section 1, $H_{(m)}^{\prime} \leq H^{\prime} \cap L_{n}^{\prime} \leq L_{n}^{\prime}$. By (1c), each $H^{\prime}$ is isomorphic to one of the groups $\Gamma_{v}$, so $H^{\prime}$ is finitely generated (Data 5.1). Hence, by Lemma 1.2, there is an $r \geq m$ such that, for every $H^{\prime} \in \mathcal{G}^{\prime}$, every automorphism of $\left(H^{\prime}\right)^{(m)}$ which lifts to an automorphism of $\left(H^{\prime}\right)^{(r)}$ can be lifted to an automorphism of $H^{\prime}$. By Lemma 3.1(a) there is an open normal subgroup $\hat{L}^{\prime}$ of $G^{\prime}$ such that $H^{\prime} \cap \hat{L}^{\prime} \leq H_{(r)}^{\prime}$ for every $H^{\prime} \in \mathcal{G}^{\prime}$. We may assume that $\hat{L}^{\prime} \leq L_{n}^{\prime}$. This gives the following diagram in which all horizontal maps are quotient maps.

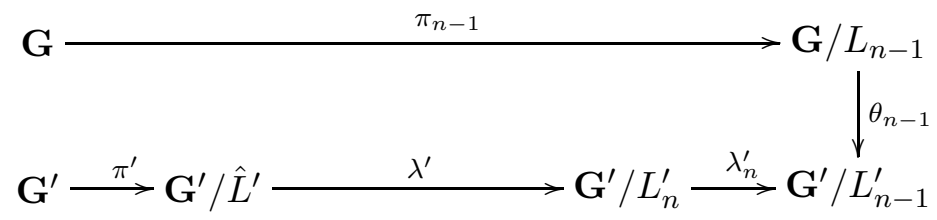

In particular, $\lambda^{\prime} \circ \pi^{\prime}=\pi_{n}^{\prime}$ and $\lambda_{n}^{\prime} \circ \lambda^{\prime} \circ \pi^{\prime}=\pi_{n-1}^{\prime}$.

By $\left(3^{\prime} \mathrm{d}\right)$,

$$
\left(\theta_{n-1} \circ \pi_{n-1}: \mathbf{G} \rightarrow \mathbf{G}^{\prime} / L_{n-1}^{\prime}, \lambda_{n}^{\prime} \circ \lambda^{\prime}: \mathbf{G}^{\prime} / \hat{L}^{\prime} \rightarrow \mathbf{G}^{\prime} / L_{n-1}^{\prime}\right)
$$

is a finite locally solvable embedding problem. Hence, by $(1 \mathrm{~d})$, there exists an epimorphism $\tau: \mathbf{G} \rightarrow \mathbf{G}^{\prime} / \hat{L}^{\prime}$ such that $\lambda_{n}^{\prime} \circ \lambda^{\prime} \circ \tau=\theta_{n-1} \circ \pi_{n-1}$. 
Let $\hat{L}$ be the kernel of $\tau: \mathbf{G} \rightarrow \mathbf{G}^{\prime} / \hat{L}^{\prime}$ and let $L_{n}$ be the kernel of $\lambda^{\prime} \circ \tau: \mathbf{G} \rightarrow \mathbf{G}^{\prime} / L_{n}^{\prime}$. Then $\tau, \lambda^{\prime} \circ \tau$ induce isomorphisms $\hat{\theta}: \mathbf{G} / \hat{L} \rightarrow \mathbf{G}^{\prime} / \hat{L}^{\prime}$ and $\theta_{n}: \mathbf{G} / L_{n} \rightarrow \mathbf{G}^{\prime} / L_{n}^{\prime}$ such that the following diagram commutes.

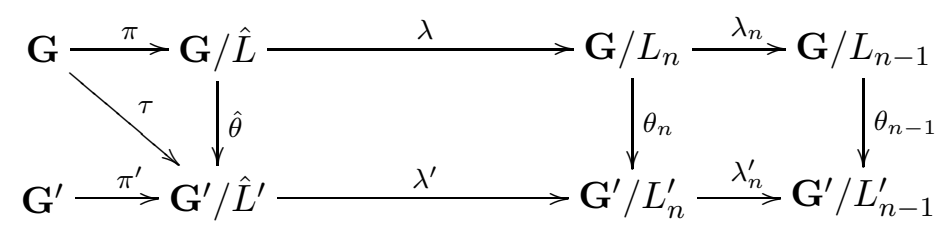

This gives (3c).

Now we verify (3d). Since $\tau\left(\mathcal{G}_{v}\right)=\pi^{\prime}\left(\mathcal{G}_{v}^{\prime}\right)$, the following condition holds for each $v \in S$ : for each $H \in \mathcal{G}_{v}$ there is an $H^{\prime} \in \mathcal{G}_{v}^{\prime}$ and for each $H^{\prime} \in \mathcal{G}_{v}^{\prime}$ there is $H \in \mathcal{G}_{v}$ with $\hat{\theta}\left((\pi(H))=\pi^{\prime}\left(H^{\prime}\right)\right.$. For such groups $\hat{\theta}$ induces an isomorphism $H / H \cap \hat{L} \cong H^{\prime} / H^{\prime} \cap \hat{L}^{\prime}$. Thus we have the following diagram

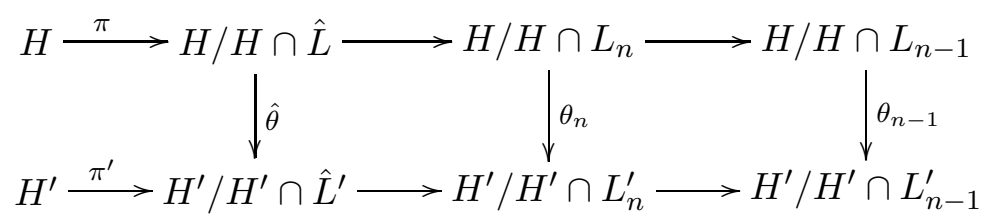

in which the horizontal maps are the quotient maps and $\hat{\theta}, \theta_{n}, \theta_{n-1}$ are the restrictions of these maps defined above to the images of $H$.

We have $H^{\prime} \cap \hat{L}^{\prime} \leq H_{(r)}^{\prime} \leq H_{(m)}^{\prime} \leq H^{\prime} \cap L_{n}^{\prime}$. Hence, by Corollary 1.3, $\theta_{n}$ lifts to an isomorphism $H \rightarrow H^{\prime}$.

If (3a) holds, then we are done. If not, we replace $L_{n-1}, L_{n-1}^{\prime}$, and $\theta_{n-1}$ by $L_{n}^{\prime}, L_{n}$, and $\theta_{n}^{-1}$. Reversing the roles of $\mathbf{G}$ and $\mathbf{G}^{\prime}$ in the above construction, we may construct an open normal subgroup $M_{n}$ of $G$ in $L_{n} \cap K_{n}$, an open normal subgroup $M_{n}^{\prime}$ of $G^{\prime}$ in $L_{n}^{\prime}$ (hence in $K_{n}^{\prime}$ ) and an isomorphism $\mu_{n}: \mathbf{G}^{\prime} / M_{n}^{\prime} \rightarrow \mathbf{G} / M_{n}$ such that the following diagram (in which the horizontal arrows are the quotient maps) commutes

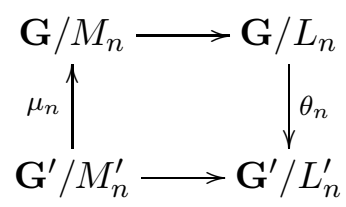

and where (3d) holds with respect to the quotient maps $\mathbf{G}^{\prime} \rightarrow \mathbf{G}^{\prime} / M_{n}^{\prime}$ and $\mathbf{G} \rightarrow \mathbf{G} / M_{n}$ and to $\mu_{n}$ replacing $\theta_{n}$. Finally, we replace $L_{n}, L_{n}^{\prime}$ and $\theta_{n}$ by $M_{n}, M_{n}^{\prime}$, and $\mu_{n}^{-1}$, respectively to obtain all conditions of (3).

This finishes the induction. 
Proposition 6.4. Let $\left(S, S_{0}, S_{1}, \Gamma_{v}, T_{v}\right)_{v \in S}$ be as in Data 5.1 , let $\mathbf{G}=$ $\left(G, \mathcal{G}_{v}\right)_{v \in S}$ be a Cantor group pile over $\left(\Gamma_{v}\right)_{v \in S}$, and let $G_{0} \in \mathcal{G}_{0}$. Suppose $T_{v}$ is a homeomorphic copy of the Cantor space, $v \in S$. Then:

(a) $G$ is isomorphic to the free product of the semi-constant sheaf $\mathbf{X}=$ $(X, \mathrm{pr}, T)$, where $X=\bigcup_{v \in S} \Gamma_{v} \times T_{v}, T=\bigcup_{v \in S} T_{v}$, and pr is the projection on the second coordinate.

(b) $G$ has a presentation as an inner free product

$$
G=G_{0} * \prod_{v \in S_{1}} \prod_{t \in T_{v}} G_{t}
$$

such that for each $v \in S_{1}$ the set $\left\{G_{t} \mid t \in T_{v}\right\}$ is a closed system of representatives of the $G$-classes of $\mathcal{G}_{v}$.

Proof of (a). By Corollary 6.2, $\mathbf{G}_{T}$ is a Cantor group pile. By assumption, so is G. Hence, by Proposition 6.3, there is an isomorphism $\theta: \mathbf{G}_{T} \rightarrow \mathbf{G}$ of group piles. By construction, $G_{T}$ is the free product of the sheaf $\mathbf{X}$, hence so is $G$.

Proof of (b). By (a), $\theta\left(G_{T, 0}\right)=G_{0}^{g}$ for some $g \in G$. Let $\theta^{\prime}=\iota_{g^{-1}} \circ \theta$ be the composition of $\theta$ with conjugation by $g^{-1}$. Then $\theta^{\prime}: \mathbf{G}_{T} \rightarrow \mathbf{G}$ is an isomorphism of group piles satisfying $\theta^{\prime}\left(G_{T, 0}\right)=G_{0}$. Replacing $\theta$ by $\theta^{\prime}$, we may assume that $\theta\left(G_{T, 0}\right)=G_{0}$. Now let $G_{t}=\theta\left(G_{T, t}\right)$ for each $t \in T_{1}$.

By (3) of Section $5, G_{T}=G_{T, 0} * *_{v \in S_{1}} *_{t \in T_{v}} G_{T, t}$, so $G=G_{0} *$ $\mathbb{F}_{v \in S_{1}} \mathbb{F}_{t \in T_{v}} G_{t}$. Moreover, for each $v \in S_{1},\left\{G_{T, t} \mid t \in T_{v}\right\}$ is a closed system of representatives of the $G_{T}$-classes of $\mathcal{G}_{T, v}$ (Proposition $5.3(\mathrm{~g})$ ). Since $\theta\left(\mathcal{G}_{T, v}\right)=\mathcal{G}_{v}$, the set $\left\{G_{t} \mid t \in T_{v}\right\}$ is a closed system of representatives of the $G$-classes of $\mathcal{G}_{v}$.

\section{$\S 7$. Big quotients}

Omitting the spaces $T_{v}$ from Data 5.1 , we demand that the set $\left\{\Gamma_{v} \mid\right.$ $\left.v \in S_{1}\right\}$ has a "system of big quotients". Big quotients enter in an essential way in the proof of Proposition 7.5 which is one of the key steps in the proof of our main result.

DATA 7.1. We continue to consider the finite set $S=S_{0} \cup S_{1}$ with $S_{0}=\{0\}$ and $1 \notin S$. For each $v \in S$ let $\Gamma_{v}$ be a finitely generated profinite group. Put $\mathcal{C}_{1}=\left\{\Gamma_{v} \mid v \in S_{1}\right\}$. A finite quotient $\bar{\Gamma}_{v}$ of $\Gamma_{v}$ is said to be big if it satisfies the following condition: 
(1) Let $\hat{F}$ be a finitely generated free profinite group and $J$ a finite set. For each $j \in J$ let $\Delta_{j} \in \mathcal{C}_{1}$. Consider the free profinite product $B^{*}=$ $\hat{F} * \mathbb{F}_{j \in J} \Delta_{j}$. Let $\Delta$ be a closed subgroup of $B^{*}$ with epimorphisms $\Gamma_{v} \stackrel{\gamma}{\longrightarrow} \Delta \rightarrow \bar{\Gamma}_{v}$. Then $\Delta$ is conjugate in $B^{*}$ to a closed subgroup of a certain $\Delta_{j}$ and $\gamma$ is an isomorphism.

The definition of "big quotients" depends on $\mathcal{C}_{1}$. The latter set will be always clear from the context.

Note that if $\bar{\Gamma}^{\prime}$ is a finite quotient of $\Gamma_{v}$ and $\bar{\Gamma}_{v}$ is a quotient of $\bar{\Gamma}^{\prime}$, then also $\bar{\Gamma}^{\prime}$ is a big quotient of $\Gamma_{v}$.

We assume that

(2a) $\Gamma_{0}$ is a finitely generated free profinite group and that

(2b) each $\Gamma_{v}$ with $v \in S_{1}$ has a big quotient $\bar{\Gamma}_{v}$.

Lemma 7.2. Let $\mathbf{B}=\left(B, \mathcal{B}_{v}\right)_{v \in S}$ be a finite group pile. Suppose each group in $\mathcal{B}_{v}$ is a quotient of $\Gamma_{v}$. Then there exists a finite group pile $\mathbf{B}^{\prime}=$ $\left(B^{\prime}, \mathcal{B}_{v}^{\prime}\right)_{v \in S}$ and an epimorphism $\beta: \mathbf{B}^{\prime} \rightarrow \mathbf{B}$ such that the following holds for each $v \in S$ :

(a) For every homomorphism $\psi: \Gamma_{v} \rightarrow B$ with $\psi\left(\Gamma_{v}\right) \in \mathcal{B}_{v}$ there is a homomorphism $\psi^{\prime}: \Gamma_{v} \rightarrow B^{\prime}$ with $\psi^{\prime}\left(\Gamma_{v}\right) \in \mathcal{B}_{v}^{\prime}$ and $\beta \circ \psi^{\prime}=\psi$.

(b) Suppose $v \in S_{1}$. If a subgroup $C^{\prime}$ of $B^{\prime}$ is a quotient of $\Gamma_{v}$ and $\beta\left(C^{\prime}\right)$ is a big quotient of $\Gamma_{v}$, then $\beta\left(C^{\prime}\right)$ is a subgroup of some group in $\mathcal{B}_{1}$.

Moreover, if $\mathbf{B}$ is deficient, then $\mathbf{B}^{\prime}$ can be chosen to be deficient.

Proof. We divide the proof into three parts.

PART A: Free product. Choose a homomorphism $\psi_{0}: \Gamma_{0} \rightarrow B$ such that $\psi_{0}\left(\Gamma_{0}\right) \in \mathcal{B}_{0}$, write $\Gamma_{0}$ also as $\Gamma^{\left(\psi_{0}\right)}$, and let $\Psi_{0}=\left\{\psi_{0}\right\}$. For each $v \in S_{1}$ let $\Psi_{v}$ be the set of all homomorphisms $\psi: \Gamma_{v} \rightarrow B$ such that $\psi\left(\Gamma_{v}\right) \in \mathcal{B}_{1}$. For each $\psi \in \Psi_{v}$ let $\Gamma^{(\psi)}$ be an identical copy of $\Gamma_{v}$. Then $\psi$ is a homomorphism of $\Gamma^{(\psi)}$ into $B$ whose image lies in $\mathcal{B}_{1}$. Since $\Gamma_{v}$ is finitely generated and $B$ is finite, the set $\Psi_{v}$ is finite. We consider the various $\Psi_{v}$ as disjoint and set $\Psi=\bigcup_{v \in S} \Psi_{v}$. Finally consider a finitely generated free profinite group $\hat{F}$ with $\operatorname{rank}(\hat{F}) \geq \operatorname{rank}(B)$ and let $\zeta: \hat{F} \rightarrow B$ be an epimorphism.

Now consider the free product $B^{(\infty)}=\hat{F} * \circledast_{v \in S} \circledast_{\psi \in \Psi_{v}} \Gamma^{(\psi)}$ and let $\gamma: B^{(\infty)} \rightarrow B$ be the epimorphism whose restriction to $\hat{F}$ is $\zeta$, and to each 
$\Gamma^{(\psi)}$ is $\psi$. Choose a descending sequence $\operatorname{Ker}(\gamma)=N^{(0)} \geq N^{(1)} \geq N^{(2)} \geq$ ... of open normal subgroups of $B^{(\infty)}$ whose intersection is 1 . For each $j \geq 0$ put $B^{(j)}=B^{(\infty)} / N^{(j)}$. (We are not using here $B^{(j)}$ in the sense of Section 1.) Then let $\gamma^{(j)}: B^{(\infty)} \rightarrow B^{(j)}, \beta^{(j)}: B^{(j)} \rightarrow B$, and $\gamma_{j+1, j}: B^{(j+1)} \rightarrow B^{(j)}$ be the quotient maps. Then $\gamma^{(j)}=\gamma_{j+1, j} \circ \gamma^{(j+1)}$ and $\beta \circ \gamma^{(j)}=\gamma$. Since $\gamma$ is an epimorphism, so is each $\beta^{(j)}$. We may identify $B$ with $B^{(0)}$ and $\gamma$ with $\gamma^{(0)}$.

PArt B: Construction of $B^{\prime}$ and Proof of (b). We choose $B^{\prime}$ to be $B^{(j)}$ with $j$ sufficiently large. To that end we consider $v \in S_{1}$ and a subgroup $C$ of $B$ which is a big quotient of $\Gamma_{v}$ but contained in no group belonging to $\mathcal{B}_{1}$. Assume, toward contradiction, that for each $j$ there is a subgroup $C^{(j)}$ of $B^{(j)}$ which is a quotient of $\Gamma_{v}$ such that $\beta^{(j)}\left(C^{(j)}\right)=C$. Since for each $j$ the group $B^{(j)}$ has only finitely many subgroups, a compactness argument allows us to choose the $C^{(j)}$ 's such that $\gamma_{j+1, j}\left(C^{(j+1)}\right)=C^{(j)}$ for all $j$. The inverse image of the $C^{(j)}$ 's is a closed subgroup $C^{(\infty)}$ of $B^{(\infty)}$ satisfying $\gamma^{(j)}\left(C^{(\infty)}\right)=$ $C^{(j)}$ for each $j$. In particular, $\gamma\left(C^{(\infty)}\right)=C$. Since $\Gamma_{v}$ is finitely generated, another compactness argument gives a compatible sequence of epimorphisms $\delta^{(j)}: \Gamma_{v} \rightarrow C^{(j)}$. That sequence defines an epimorphism $\delta: \Gamma \rightarrow C^{(\infty)}$. Note that $B^{(\infty)}=\left(\hat{F} * \Gamma^{(0)}\right) * \Gamma_{v \in S_{1}} \circledast_{\psi \in \Psi_{v}} \Gamma^{(\psi)}$ and $\hat{F} * \Gamma^{(0)}$ is a finitely generated free profinite group. By the defining properties of $\mathcal{C}_{1}$ (Data 7.1), $C^{(\infty)}$ is conjugate to a closed subgroup of $\Gamma^{(\psi)}$ for some $\psi \in \Psi_{v}$ and $v \in S_{1}$. Then $C=\gamma\left(C^{(\infty)}\right)$ is conjugate to a subgroup of $\gamma\left(\Gamma^{(\psi)}\right)=\psi\left(\Gamma^{(\psi)}\right) \in \mathcal{B}_{1}$. Since $\mathcal{B}_{1}$ is closed under conjugation, $C$ is contained in a group in $\mathcal{B}_{1}$, a contradiction.

The contradiction proves that there exists a positive integer $j$ such that (b) holds for $B^{\prime}=B^{(j)}$ and $\beta=\beta^{(j)}$.

PART C: Proof of (a). For each $v \in S$ let $\mathcal{B}_{v}^{\prime}$ be the conjugacy domain of $\operatorname{Subgr}\left(B^{\prime}\right)$ generated by the groups $\gamma^{(j)}\left(\Gamma^{(\psi)}\right)$ with $\psi \in \Psi_{v}$. If $\mathbf{B}$ is deficient, we choose $\mathcal{B}_{0}^{\prime}$ to be the conjugacy class consisting of the trivial group.

Let $\psi: \Gamma_{0} \rightarrow B$ be a homomorphism with $\psi\left(\Gamma_{0}\right) \in \mathcal{B}_{0}$. Then with $B_{0}=\gamma\left(\Gamma^{\left(\psi_{0}\right)}\right)$ and $B_{0}^{\prime}=\gamma^{(j)}\left(\Gamma^{\left(\psi_{0}\right)}\right)$ (or $B_{0}=B_{0}^{\prime}=1$ if $\mathbf{B}$ is deficient), we have $\psi\left(\Gamma_{0}\right)=B_{0}^{b}$ for some $b \in B$. Choose $b^{\prime} \in B^{\prime}$ such that $\beta\left(b^{\prime}\right)=b$. Then, $B_{0}^{\prime} \in \mathcal{B}_{0}^{\prime}, \operatorname{rank}\left(\left(B_{0}^{\prime}\right)^{b^{\prime}}\right)=\operatorname{rank}\left(B_{0}^{\prime}\right) \leq \operatorname{rank}\left(\Gamma_{0}\right)$, and $\beta\left(\left(B_{0}^{\prime}\right)^{b^{\prime}}\right)=$ $B_{0}^{b}=\psi\left(\Gamma_{0}\right)$. By assumption, $\Gamma_{0}$ is a finitely generated free profinite group. Therefore, by Gaschütz, there exists an epimorphism $\psi^{\prime}: \Gamma_{0} \rightarrow\left(B_{0}^{\prime}\right)^{b^{\prime}}$ such that $\beta \circ \psi^{\prime}=\psi$ [FrJ, Prop. 17.7.3]. This settles the case $v=0$.

Now consider $v \in S_{1}$ and let $\psi: \Gamma_{v} \rightarrow B$ be a homomorphism with 
$\psi\left(\Gamma_{v}\right) \in \mathcal{B}_{v}$. Then $\psi \in \Psi_{v}$ and $\Gamma_{v}=\Gamma^{(\psi)}$. Set $\psi^{\prime}=\left.\gamma^{(j)}\right|_{\Gamma^{(\psi)}}$. Then $\psi^{\prime}\left(\Gamma_{v}\right) \in \mathcal{B}_{v}^{\prime}$ and $\beta \circ \psi^{\prime}=\psi$, as desired.

Remark 7.3. Non-improvable. It is impossible to deduce in Lemma 7.2(b) that $\beta\left(C^{\prime}\right)$ is a subgroup of some group in $\mathcal{B}_{v}$ because, for example, $\Gamma_{v}$ can be isomorphic to a subgroup of $\Gamma_{v^{\prime}}$ for distinct $v, v^{\prime} \in S_{1}$. We overcome this difficulty in Part $\mathrm{F}$ of the proof of Proposition 7.5 by considering separated rigid finite embedding problems.

Lemma 7.4. Let $\mathbf{G}=\left(G, \mathcal{G}_{v}\right)_{v \in S}$ be a separated group pile. Suppose each group in $\mathcal{G}_{v}$ is isomorphic to $\Gamma_{v}, v \in S_{1}$. Then there exists an open normal subgroup $K$ of $G$ with the following property: If $\varphi: G \rightarrow A$ is an epimorphism onto a finite group $A$ with $\operatorname{Ker}(\varphi) \leq K$, then $\mathbf{A}=\left(A, \varphi\left(\mathcal{G}_{v}\right)\right)_{v \in S}$ is separated and $\varphi(H)$ is a big quotient of $\Gamma_{v}$ for every $H \in \mathcal{G}_{v}$ and each $v \in S_{1}$.

Proof. There is an $n$ such that, in the notation of Section $1, \Gamma_{v}^{(n)}$ is a big quotient of $\Gamma_{v}$ for each $v \in S_{1}$. Thus, $H^{(n)}$ is a big quotient of $H$ for each $H \in \mathcal{G}_{1}$. Note that the groups in $\mathcal{G}_{0}$ are conjugate to each other, hence isomorphic. Lemma 3.1 gives an open normal subgroup $K$ of $G$ such that $\mathbf{G} / K$ is separated and $K \cap H \leq H_{(n)}$ for each $H \in \mathcal{G}_{1}$. Consider an epimorphism $\varphi: G \rightarrow A$ with $A$ finite and $\operatorname{Ker}(\varphi) \leq K$. Let $H \in \mathcal{G}_{1}$. Then $H \rightarrow H^{(n)}$ factors through $\varphi: H \rightarrow \varphi(H)$, hence $\varphi(H)$ is a big quotient of $H$.

The following proposition is an essential step toward a solution of a finite locally solvable embedding problem (3) for a group pile $\mathbf{G}$. We cover the deficient group pile associated with $\mathbf{G}$ by a deficient group pile $\mathbf{H}$ and solve the corresponding embedding problem (4) for $\mathbf{H}$ assuming among others that the group theoretic embedding problem for the underlying profinite groups is solvable.

Proposition 7.5. Let $\mathbf{G}=\left(G, \mathcal{G}_{v}\right)_{v \in S}$ be a separated deficient group pile, $\mathbf{H}=\left(H, \mathcal{H}_{v}\right)_{v \in S}$ a deficient group pile, and $\lambda: \mathbf{H} \rightarrow \mathbf{G}$ a rigid epimorphism. Suppose:

(a) Each group in $\mathcal{G}_{v}$ is isomorphic to $\Gamma_{v}, v \in S_{1}$.

(b) There are no inclusions between distinct groups in $\mathcal{G}_{1}$.

(c) $G$ is $\mathcal{G}_{1}$-projective. 
(d) The space $\overline{\mathcal{G}}_{1}$ of the $G$-orbits of $\mathcal{G}_{1}$ has no isolated points.

(e) For every finite split embedding problem $(\varphi: G \rightarrow A, \alpha: B \rightarrow A)$ of profinite groups there exists a group epimorphism $\delta: H \rightarrow B$ such that $\alpha \circ \delta=\varphi \circ \lambda$ and $\lambda(\operatorname{Ker}(\delta))=\operatorname{Ker}(\varphi)$.

Then for every finite locally solvable embedding problem

$$
(\varphi: \mathbf{G} \rightarrow \mathbf{A}, \alpha: \mathbf{B} \rightarrow \mathbf{A})
$$

of deficient group piles there exists an epimorphism $\delta: \mathbf{H} \rightarrow \mathbf{B}$ of deficient group piles such that $\alpha \circ \delta=\varphi \circ \lambda$ and $\lambda(\operatorname{Ker}(\delta))=\operatorname{Ker}(\varphi)$.

Proof. Let (3) be a finite locally solvable embedding problem of deficient group piles. We want to solve the embedding problem

$$
(\varphi \circ \lambda: \mathbf{H} \rightarrow \mathbf{A}, \alpha: \mathbf{B} \rightarrow \mathbf{A})
$$

Let us call a group epimorphism $\delta: H \rightarrow B$ which satisfies $\alpha \circ \delta=\varphi \circ \lambda$ and $\lambda(\operatorname{Ker}(\delta))=\operatorname{Ker}(\varphi)$ a group theoretic regular solution of (4). It will be a regular solution if $\delta\left(\mathcal{H}_{v}\right)=\mathcal{B}_{v}$ for each $v \in S_{1}$.

PART A: Domination principle. If

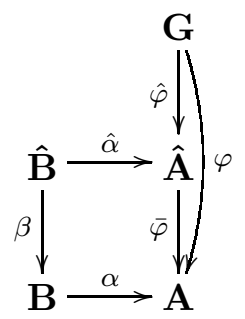

is a commutative diagram of epimorphisms of deficient group piles with $\hat{B}=B \times_{A} \hat{A}$, then

$$
(\hat{\varphi} \circ \lambda: \mathbf{H} \rightarrow \hat{\mathbf{A}}, \hat{\alpha}: \hat{\mathbf{B}} \rightarrow \hat{\mathbf{A}})
$$

is a finite embedding problem dominating (4). If $\hat{\delta}$ is a (group theoretic) regular solution of (6), then $\delta=\beta \circ \hat{\delta}$ is a (group theoretic) regular solution to $(4)$.

Indeed, suppose that $\hat{\alpha} \circ \hat{\delta}=\hat{\varphi} \circ \lambda$ and $\lambda(\operatorname{Ker}(\hat{\delta}))=\operatorname{Ker}(\hat{\varphi})$. We prove that $\lambda(\operatorname{Ker}(\delta))=\operatorname{Ker}(\varphi)$. If $g \in \lambda(\operatorname{Ker}(\delta))$, then $g=\lambda(h)$ with $h \in \operatorname{Ker}(\delta)$. Hence, $\varphi(g)=\varphi(\lambda(h))=\alpha(\delta(h))=1$. Conversely, suppose 
that $g \in \operatorname{Ker}(\varphi)$. Choose $h \in H$ with $\lambda(h)=g$ and set $\hat{b}=\hat{\delta}(h)$. Then $\alpha(\beta(\hat{b}))=\varphi(\lambda(h))=\varphi(g)=1$. By [FrJ, Lemma 22.2.4] we may write $\hat{b}=\hat{b}_{1} \hat{b}_{2}$ with $\beta\left(\hat{b}_{1}\right)=1$ and $\hat{\alpha}\left(\hat{b}_{2}\right)=1$. Choose $h_{1} \in H$ with $\hat{\delta}\left(h_{1}\right)=\hat{b}_{1}$ and set $g_{1}=\lambda\left(h_{1}\right)$. Then $\hat{\varphi}\left(g_{1}^{-1} g\right)=\hat{\varphi}\left(\lambda\left(h_{1}^{-1} h\right)\right)=\hat{\alpha}\left(\hat{\delta}\left(h_{1}^{-1} h\right)\right)=\hat{\alpha}\left(\hat{b}_{1}^{-1} \hat{b}\right)=$ $\hat{\alpha}\left(\hat{b}_{2}\right)=1$. Since $\lambda(\operatorname{Ker}(\hat{\delta}))=\operatorname{Ker}(\hat{\varphi})$, there exists $h_{2} \in \operatorname{Ker}(\hat{\delta})$ with $g_{1}^{-1} g=$ $\lambda\left(h_{2}\right)$. Thus, $g=\lambda\left(h_{1} h_{2}\right)$ and $\delta\left(h_{1} h_{2}\right)=\beta\left(\hat{\delta}\left(h_{1}\right)\right) \beta\left(\hat{\delta}\left(h_{2}\right)\right)=\beta\left(\hat{b}_{1}\right) \beta(1)=1$. Consequently, $g \in \lambda(\operatorname{Ker}(\delta))$, as claimed.

Since fiber products over $\hat{\alpha}: \hat{B} \rightarrow \hat{A}$ are fiber products over $\alpha: B \rightarrow A$, we may iterate the same construction several times.

Similarly, if $\beta: B^{\prime} \rightarrow B$ is an epimorphism of profinite groups, $\delta^{\prime}: H \rightarrow$ $B^{\prime}$ is a homomorphism satisfying $\alpha \circ \beta \circ \delta^{\prime}=\varphi \circ \lambda$, and $\delta=\beta \circ \delta^{\prime}$, then $\lambda\left(\operatorname{Ker}\left(\delta^{\prime}\right)\right)=\operatorname{Ker}(\varphi)$ implies $\lambda(\operatorname{Ker}(\delta))=\operatorname{Ker}(\varphi)$.

PART B: Without loss A is separated, $\varphi\left(G_{v}\right)$ is a big quotient of $\Gamma_{v}$ for each $G_{v} \in \mathcal{G}_{v}$ and each $v \in S_{1}$, and $\alpha$ is rigid. Indeed, by Lemma 7.4 (here we use Assumption (a)) and Lemma 4.3 there is a commutative diagram (5) of deficient group piles such that $\hat{\mathbf{A}}$ is separated, $\hat{\varphi}\left(G_{v}\right)$ is a big quotient of $\Gamma_{v}$ for each $G_{v} \in \mathcal{G}_{v}$ and each $v \in S_{1}, \hat{B}=B \times_{A} \hat{A}$, and $\hat{\alpha}$ is rigid. By Part A, a regular solution $\hat{\delta}: \mathbf{H} \rightarrow \hat{\mathbf{B}}$ of (6) gives a regular solution $\beta \circ \hat{\delta}: \mathbf{H} \rightarrow \mathbf{B}$ of (4).

PART C: For every locally embedding problem (3) of deficient group piles, embedding problem (4) has a group theoretic regular solution. In fact, Lemma 4.3 gives a commutative diagram (5) in which $\hat{\alpha}$ splits (here we use Assumption (c)). Assumption (e) gives a group theoretic regular solution $\hat{\delta}$ to (6). By Part A, $\delta=\beta \circ \hat{\delta}$ is a group theoretic regular solution of $(4)$.

PART D: Embedding problem (4) has a group theoretic regular solution $\delta: H \rightarrow B$ such that for each $H_{1} \in \mathcal{H}_{1}$ there is a $B_{1} \in \mathcal{B}_{1}$ with $\delta\left(H_{1}\right) \leq B_{1}$. Indeed, by Lemma 7.2 there exists an epimorphism $\beta: \mathbf{B}^{\prime} \rightarrow \mathbf{B}$ of deficient group piles such that (a) and (b) of that lemma hold. Let $G_{v} \in \mathcal{G}_{v}$ for some $v \in S_{1}$. Then there exists $B_{v} \in \mathcal{B}_{v}$ and an epimorphism $\psi: G_{v} \rightarrow$ $B_{v}$ such that $\alpha \circ \psi=\left.\varphi\right|_{G_{v}}$ (because (3) is locally solvable). By (a) of Lemma 7.2, $\psi$ lifts to an epimorphism $\psi^{\prime}: G_{v} \rightarrow B_{v}^{\prime}$ for some $B_{v}^{\prime} \in \mathcal{B}_{v}^{\prime}$ with $\beta\left(B_{v}^{\prime}\right)=B_{v}$. Thus $G_{v}$ is compatible with $B_{v}^{\prime}$. Therefore $\mathcal{B}_{v}^{\prime \prime}=\left\{B_{v}^{\prime} \in\right.$ $\mathcal{B}_{v}^{\prime} \mid B_{v}^{\prime}$ is compatible with some $\left.G_{v} \in \mathcal{G}_{v}\right\}$ is a $B^{\prime}$-domain that satisfies $\beta\left(\mathcal{B}_{v}^{\prime \prime}\right)=\mathcal{B}_{v}$. If necessary, replace $\mathcal{B}_{v}^{\prime}$ by $\mathcal{B}_{v}^{\prime \prime}$ to make $(\varphi, \alpha \circ \beta)$ a locally solvable embedding problem for $\mathbf{G}$.

Now consider $H_{1} \in \mathcal{H}_{1}$. Put $G_{1}=\lambda\left(H_{1}\right), B_{1}^{\prime}=\delta^{\prime}\left(H_{1}\right)$, and $B_{1}=$ 
$\beta\left(B_{1}^{\prime}\right)$. Then $B_{1}=\delta\left(H_{1}\right)$ and $\alpha\left(B_{1}\right)=\varphi\left(G_{1}\right)$. Since $\lambda$ is rigid, its restriction to $H_{1}$ is an isomorphism $H_{1} \rightarrow G_{1}$. Therefore $B_{1}^{\prime} \leq B^{\prime}$ is a quotient of $G_{1} \in \mathcal{G}_{1}$. By Part B, $\varphi\left(G_{1}\right)$ is a big quotient of $\Gamma_{v}$ for the unique $v \in S_{1}$ with $G_{1} \in \mathcal{G}_{v}$. By Lemma $7.2(\mathrm{~b}), B_{1}$ is a subgroup of some group in $\mathcal{B}_{1}$, as claimed.

PART E: Embedding problem (4) has a group theoretic regular solution $\delta: H \rightarrow B$ such that $\delta\left(\mathcal{H}_{1}\right) \subseteq \mathcal{B}_{1}$. By Lemma 3.2 and Lemma 4.3 , there is a commutative diagram (5) with $\hat{B}=B \times_{A} \hat{A}$ in which $\hat{\alpha}$ is rigid such that

(7) if $\hat{A}_{1}, \hat{A}_{2} \in \hat{\mathcal{A}}_{1}$ and $\hat{A}_{1} \leq \hat{A}_{2}$, then $\bar{\varphi}\left(\hat{A}_{1}\right)=\bar{\varphi}\left(\hat{A}_{2}\right)$

(here we use Assumption (b)). By Part D, the embedding problem

$$
(\hat{\varphi} \circ \lambda: \mathbf{H} \rightarrow \hat{\mathbf{A}}, \hat{\alpha}: \hat{\mathbf{B}} \rightarrow \hat{\mathbf{A}})
$$

has a group theoretic regular solution $\hat{\delta}: H \rightarrow \hat{B}$ such that for each $H_{1} \in \mathcal{H}_{1}$ there is $\hat{B}_{1} \in \hat{\mathcal{B}}_{1}$ with $\hat{\delta}\left(H_{1}\right) \leq \hat{B}_{1}$. Fix such $H_{1}, \hat{B}_{1}$. Then

$$
\hat{\alpha}\left(\hat{\delta}\left(H_{1}\right)\right) \leq \hat{\alpha}\left(\hat{B}_{1}\right) \quad \text { and } \quad \beta\left(\hat{\delta}\left(H_{1}\right)\right) \leq \beta\left(\hat{B}_{1}\right) .
$$

But $\hat{\alpha}\left(\left(\hat{\delta}\left(H_{1}\right)\right)=\hat{\varphi}\left(\lambda\left(H_{1}\right)\right) \in \hat{\mathcal{A}}_{1}\right.$ and $\hat{\alpha}\left(\hat{B}_{1}\right) \in \hat{\mathcal{A}}_{1}$. Hence, by $(7)$, $\bar{\varphi}\left(\hat{\alpha}\left(\hat{\delta}\left(H_{1}\right)\right)\right)=\bar{\varphi}\left(\hat{\alpha}\left(\hat{B}_{1}\right)\right)$, that is, $\alpha\left(\beta\left(\hat{\delta}\left(H_{1}\right)\right)\right)=\alpha\left(\beta\left(\hat{B}_{1}\right)\right)$. Since $\alpha$ is rigid, hence injective on $\beta\left(\hat{B}_{1}\right) \in \mathcal{B}_{1}$, this gives $\delta\left(H_{1}\right)=\beta\left(\hat{\delta}\left(H_{1}\right)\right)=\beta\left(\hat{B}_{1}\right) \in$ $\mathcal{B}_{1}$. Consequently, $\delta=\beta \circ \hat{\delta}$ has the required property.

PART F: Embedding problem (4) has a regular solution. By (d) and by Lemma 4.5 there is a commutative diagram (5) with $\hat{B}=B \times_{A} \hat{A}$ in which $\hat{\alpha}$ is rigid such that

(9) for every $v \in S_{1}$ and $B_{v} \in \mathcal{B}_{v}$ there exists $\hat{A}_{v} \in \hat{\mathcal{A}}_{v}$ with $\alpha\left(B_{v}\right)=$ $\bar{\varphi}\left(\hat{A}_{v}\right)$ such that if $\hat{B}^{\prime} \in \hat{\mathcal{B}}_{v}$ and $\hat{\alpha}\left(\hat{B}^{\prime}\right)$ is conjugate to $\hat{A}_{v}$, then $\beta\left(\hat{B}^{\prime}\right)$ is conjugate to $B_{v}$.

By Part E, (8) has a group theoretic regular solution $\hat{\delta}: H \rightarrow \hat{B}$ such that $\hat{\delta}\left(\mathcal{H}_{1}\right) \subseteq \hat{\mathcal{B}}_{1}$. We show that the group theoretic regular solution $\delta=\beta \circ \hat{\delta}$ of (4) satisfies $\delta\left(\mathcal{H}_{v}\right)=\mathcal{B}_{v}$ for each $v \in S_{1}$. This will prove that $\delta$ is a regular solution of (4).

The inclusion $\hat{\delta}\left(\mathcal{H}_{1}\right) \subseteq \hat{\mathcal{B}}_{1}$ implies that $\delta\left(\mathcal{H}_{1}\right) \subseteq \mathcal{B}_{1}$. Let $v \in S_{1}$ and $H_{v} \in \mathcal{H}_{v}$. Then there exists $v^{\prime} \in S_{1}$ such that $\delta\left(H_{v}\right) \in \mathcal{B}_{v^{\prime}}$, so $\alpha\left(\delta\left(H_{v}\right)\right) \in$ $\mathcal{A}_{v^{\prime}}$. On the other hand, since $\varphi \circ \lambda: \mathbf{H} \rightarrow \mathbf{A}$ is an epimorphism of group piles, $\alpha\left(\delta\left(H_{v}\right)\right)=\varphi\left(\lambda\left(H_{v}\right)\right) \in \mathcal{A}_{v}$. Therefore $\mathcal{A}_{v} \cap \mathcal{A}_{v^{\prime}} \neq \emptyset$. But, by Part B, A is separated, so $v=v^{\prime}$. Consequently, $\delta\left(\mathcal{H}_{v}\right) \subseteq \mathcal{B}_{v}$. 
Conversely, let $B_{v} \in \mathcal{B}_{v}$. Let $\hat{A}_{v} \in \hat{\mathcal{A}}_{v}$ be as in (9). Then, there is an $H_{v} \in \mathcal{H}_{v}$ such that $\hat{\varphi} \circ \lambda\left(H_{v}\right)=\hat{A}_{v}$. Since $\hat{\delta}\left(\mathcal{H}_{1}\right) \subseteq \hat{\mathcal{B}}_{1}$, we have $\hat{\delta}\left(H_{v}\right) \in \hat{\mathcal{B}}_{v^{\prime}}$ for some $v^{\prime} \in S_{1}$. Thus, $\hat{\alpha}\left(\hat{\delta}\left(H_{v}\right)\right) \in \hat{\mathcal{A}}_{v^{\prime}}$ and $\hat{\varphi}\left(\lambda\left(H_{v}\right)\right) \in \hat{\mathcal{A}}_{v}$. Since $\hat{\alpha} \circ \hat{\delta}=\lambda \circ \hat{\varphi}$, we get $\hat{\mathcal{A}}_{v} \cap \hat{\mathcal{A}}_{v^{\prime}} \neq \emptyset$. Since $\mathbf{A}$ is separated, so is $\hat{\mathbf{A}}$. Therefore, $v=v^{\prime}$. Finally, since $\hat{\delta}\left(H_{v}\right)=\hat{A}_{v}$, Condition (9) gives a $b \in B$ with $\delta\left(H_{v}\right)=\beta\left(\hat{\delta}\left(H_{v}\right)\right)=B_{v}^{b}$. Let $h \in H$ with $\delta(h)=b^{-1}$. Then $H_{v}^{h} \in \mathcal{H}_{v}$ and $\delta\left(H_{v}^{h}\right)=B_{v}$.

It follows that $\delta: H \rightarrow B$ is the desired epimorphism.

Remark 7.6. Galois theoretic interpretation of regularity. Let $N / M$ be a finite Galois extension and let $t$ be transcendental over $M$. Set $G=$ $\operatorname{Gal}(M), A=\operatorname{Gal}(N / M)$, and $H=\operatorname{Gal}(M(t))$. Let $\varphi: G \rightarrow A$ and $\lambda: H \rightarrow$ $G$ be the restriction maps. Suppose $\delta$ is a group theoretic solution of (4), that is $\delta: H \rightarrow B$ is an epimorphism and $\alpha \circ \delta=\varphi \circ \lambda$. Let $P$ be the fixed field of $\operatorname{Ker}(\delta)$ in $\widetilde{M(t)}$. Then the condition $\lambda(\operatorname{Ker}(\delta))=\operatorname{Ker}(\varphi)$ for the regularity of $\delta$ is equivalent to $P \cap \tilde{M}=N$. If $\operatorname{char}(M)=0$, the latter condition is equivalent to " $P$ is regular over $N$ ".

\section{$\S 8 . \quad$ P-adically closed fields}

Ordered fields and $p$-adically valued fields have common features. For example, both have closures and the theory of these closures is model complete. In this section we present a unified vocabulary for both types of fields and survey their basic properties.

Let $(K, v)$ be an ordered field or a valued field. We call $(K, v) \mathrm{P}$-adic if

(1) either $(K, v)$ is an ordered field

(2) or $(K, v)$ is a valued field and there exists a prime number $p$ such that

(2a) the residue field of $(K, v)$ is finite, say, with $p^{f}$ elements (we call $p$ the residue characteristic),

(2b) there is a $\pi \in K^{\times}$with a smallest positive value $v(\pi)$ in $v\left(K^{\times}\right)$ (we call $\pi$ a prime element of $(K, v)$ ),

(2c) and there is a positive integer $e$ with $v(p)=e v(\pi)$ (we call $e$ the ramification index of $(K, v))$.

We refer to Case (1) as the real case and to Case (2) with $p$ the residue characteristic as the $p$-adic case. The type of $(K, v)$ is $(0,1,1)$ in the real 
case and $(p, e, f)$ in the $p$-adic case. In both cases we call $e f$ the rank of $(K, v)$.

Let $(K, v)$ and $\left(K^{\prime}, v^{\prime}\right)$ be $\mathrm{P}$-adic fields. We say that $\left(K^{\prime}, v^{\prime}\right)$ is an extension of $(K, v)$ if $K \subseteq K^{\prime}, v=\left.v^{\prime}\right|_{K}$, and in Case (2) they have the same residue characteristic. Let $(p, e, f)$ and $\left(p^{\prime}, e^{\prime}, f^{\prime}\right)$ be the types of $(K, v)$ and $\left(K^{\prime}, v^{\prime}\right)$, respectively. Then $p=p^{\prime}$, e $\mid e^{\prime}$, and $f \mid f^{\prime}$. Hence, $\left(K^{\prime}, v^{\prime}\right)$ and $(K, v)$ are of the same type if and only if they are of the same rank.

We say that $(K, v)$ is $\mathrm{P}$-adically closed if $(K, v)$ is a $\mathrm{P}$-adic field which admits no finite proper $\mathrm{P}$-adic extensions of the same type. In the real case $(K, v)$ is real closed, hence is elementarily equivalent to $(\mathbb{R}, \leq)$, where $\leq$ is the standard ordering of the $\mathbb{R}$ [Pre, Cor. 5.3]. In particular, an element $x \in K$ is nonnegative if and only if it is a square. In the $p$-adic case $K$ is elementarily equivalent to a finite extension of $\mathbb{Q}_{p}[\mathrm{HJPb}$, Prop. 8.2(j)] and $v$ is the unique valuation of $K$ such that $(K, v)$ is $\mathrm{P}$-adically closed [ $\mathrm{HJPb}$, Prop. 8.2(c)]. Occasionally, we denote $v$ also by $v_{K}$.

A P-adic closure of $(K, v)$ is an algebraic extension $(\bar{K}, \bar{v})$ of $(K, v)$ which is maximal $\mathrm{P}$-adic of the same type, in particular $(\bar{K}, \bar{v})$ is P-adically closed. Zorn's lemma guarantees the existence of $(\bar{K}, \bar{v})$. In the real case $(\bar{K}, \bar{v})$ is unique up to a $K$-isomorphism [Pre, Thm. 3.10]. This is not necessarily so in the $p$-adic case [PrR, Thm. 3.2]; however, $(\bar{K}, \bar{v})$ is Henselian [PrR, Thm. 3.1], so each Henselian closure $\left(K_{v}, v\right)$ of $(K, v)$ is $K$-embeddable in $(\bar{K}, \bar{v})$.

Each $\mathrm{P}$-adic closure of $(K, v)$ is also called a P-adic closure of $K$.

By (1) and $(2), \operatorname{char}(K)=0$. Let $K_{\text {abs }}=K \cap \tilde{\mathbb{Q}}$ be the algebraic part of $K$.

Lemma 8.1. Let $(K, v)$ be a Henselian $\mathrm{P}$-adic valued field. Then $\left(K_{\mathrm{abs}}, v\right)$ is a Henselian $\mathrm{P}$-adic field of the same type as $(K, v)$. Moreover, let $\bar{K}$ be a $\mathrm{P}$-adic closure of $K$ at $v$. Then $\bar{K}_{\mathrm{abs}}=K_{\mathrm{abs}}$. In particular, $K_{\mathrm{abs}}$ is $\mathrm{P}$-adically closed.

Proof. By [PrR, Lemma 3.5(i)], $(K, v)$ and $\left(K_{\mathrm{abs}}, v\right)$ have the same residue field. By $[\mathrm{PrR}$, Lemma $3.5(\mathrm{ii})], K_{\mathrm{abs}}$ contains a prime element of $(K, v)$. Hence, by $(2 \mathrm{c})$, the ramification indices of $(K, v)$ and $\left(K_{\mathrm{abs}}, v\right)$ are the same. It follows that $(K, v)$ and $\left(K_{\mathrm{abs}}, v\right)$ have the same type.

Now consider a P-adic closure $(\bar{K}, \bar{v})$ of $(K, v)$. Then, $(\bar{K}, \bar{v})$ has the same type as $(K, v)$, say $(p, e, f)$. By the first part of the lemma, this is also the type of $\left(\bar{K}_{\mathrm{abs}}, v\right)$. Thus, $\mathbb{Q}_{p, \text { abs }} \subseteq K_{\mathrm{abs}} \subseteq \bar{K}_{\mathrm{abs}}$ and $\left[K_{\mathrm{abs}}: \mathbb{Q}_{p, \text { abs }}\right]=$ ef $=\left[\bar{K}_{\text {abs }}: \mathbb{Q}_{p, \text { abs }}\right]$. Therefore, $K_{\text {abs }}=\bar{K}_{\text {abs }}$. 
Lemma 8.2. Let $(K, v)$ be a $\mathrm{P}$-adic field and $\left(E, v_{E}\right),\left(F, v_{F}\right)$ two $\mathrm{P}$ adic closures of $(K, v)$. Then $\left(E, v_{E}\right)$ and $\left(F, v_{F}\right)$ are elementarily equivalent as ordered fields in the real case and as valued fields in the p-adic case.

Proof. By Tarski, all real closed fields are elementarily equivalent as ordered fields [Pre, Cor. 5.3]. Suppose $(K, v)$ is $p$-adic. Replace $(K, v)$ by a Henselian closure and $\left(E, v_{E}\right),\left(F, v_{F}\right)$ by conjugate valued fields over $K$, if necessary, to assume that $(K, v)$ is Henselian. By Lemma 8.1, $E_{\text {abs }}=K_{\text {abs }}=$ $F_{\text {abs }}$ and $\left(K_{\text {abs }}, v\right)$ is P-adically closed. It follows from [PrR, Thm. 5.1] that $\left(E, v_{E}\right) \equiv\left(K_{\mathrm{abs}}, v\right) \equiv\left(F, v_{F}\right)$.

Let $F / K$ be a field extension. A $K$-rational place of $F$ is a place $\varphi$ of $F$ with residue field $K$ such that $\varphi(a)=a$ for each $a \in K$.

Lemma 8.3. Let $(K, v)$ be a $\mathrm{P}$-adic field, $(\bar{K}, \bar{v})$ a $\mathrm{P}$-adic closure of $(K, v), F$ an extension of $K$, and $\varphi$ a $K$-rational place of $F$. Then $F$ has a $\mathrm{P}$-adic closure $(\bar{F}, \bar{w})$ extending $(\bar{K}, \bar{v})$ and $\varphi$ extends to a $\bar{K}$-rational place of $\bar{F}$.

Proof. By [FrJ, Lemma 2.6.9(b)], $F$ is a regular extension of $K$. Hence, $\varphi$ extends to a $\bar{K}$-rational place $\varphi$ of $F \bar{K}$ [FrJ, Lemma 2.5.5].

Proposition 7.4(c) of [HJPa] gives an algebraic extension $\bar{F}$ of $F \bar{K}$ and $\varphi$ extends to a $\bar{K}$-rational place $\bar{\varphi}$ such that res: $\operatorname{Gal}(\bar{F}) \rightarrow \operatorname{Gal}(\bar{K})$ is an isomorphism. In the real case $\operatorname{Gal}(\bar{F})$ is of the same order of $\operatorname{Gal}(\bar{K})$, that is 2 . Hence, $\bar{F}$ is real closed. Denote the unique ordering of $\bar{F}$ by $\bar{w}$. Then $(\bar{F}, \bar{w})$ extends $(\bar{K}, \bar{v})$. In the $p$-adic case, $\bar{F}$ is P-adically closed and $\bar{F}_{\text {abs }}=\bar{K}_{\text {abs }}[$ Pop1, Thm. E11]. Denote the unique P-adic valuation of $\bar{F}$ by $\bar{w}$. Then $(\bar{F}, \bar{w})$ has the same type as $(\bar{K}, \bar{v})$ (Lemma 8.1$)$, so $(\bar{F}, \bar{w})$ extends $(\bar{K}, \bar{v})$.

LEMma 8.4. Let $K$ be a subfield of a $\mathrm{P}$-adically closed field $\bar{E}$. Then:

(a) $\bar{E} \cap \tilde{K}$ is the unique algebraic extension $\bar{K}$ of $K$ contained in $\bar{E}$ which is $\mathrm{P}$-adically closed of the same type as $\bar{E}$.

(b) In the real case let $v$ and $w$ be the unique orderings of $\bar{K}$ and $\bar{E}$; in the $p$-adic case let $v$ and $w$ be the unique $\mathrm{P}$-adic valuations of $\bar{K}$ and $\bar{E}$. Then $(\bar{K}, v)$ is an elementary submodel of $(\bar{E}, w)$.

(c) $\operatorname{Gal}(\bar{E})$ is a nontrivial finitely generated group and the map res: $\operatorname{Gal}(\bar{E}) \rightarrow \operatorname{Gal}(\bar{K})$ is an isomorphism. 
Proof of (a) and (b). Assertion (a) is [Pre, Lemma 3.13] in the real case and [PrR, Thm. 3.4] in the $p$-adic case. Assertion (b) follows from [Pre, Thm. 5.1] in the real case and from [PrR, Thm. 5.1] in the P-adic case.

Proof of (c). In the real case $\operatorname{Gal}(\bar{E}) \cong \operatorname{Gal}(\bar{K}) \cong \mathbb{Z} / 2 \mathbb{Z}$. In the $p$-adic case $\operatorname{Gal}(\bar{E})$ is infinite, e.g. because it has a finite residue field. Nevertheless $\operatorname{Gal}(\bar{E})$ is finitely generated [HJPb, Prop. $8.2(\mathrm{k})]$. Hence, by $(\mathrm{b}), \operatorname{Gal}(\bar{E}) \cong$ $\operatorname{Gal}(\bar{K})$ [FrJ, Prop. 20.4.6]. Since res: $\operatorname{Gal}(\bar{E}) \rightarrow \operatorname{Gal}(\bar{K})$ is surjective, it is an isomorphism [FrJ, Prop. 16.10.6(b)].

Each P-adic field $(K, v)$ carries a natural $v$-adic topology. If $v$ is an ordering $<$, then a basic $v$-open neighborhood of an element $a$ of $K$ is $\{x \in K \mid-\varepsilon<x-a<\varepsilon\}$, where $\varepsilon \in K$ and $\varepsilon>0$. In the $p$-adic case, a basic $v$-open neighborhood of $a$ is $\{x \in K \mid v(x-a)>v(c)\}$, where $c \in K^{\times}$.

Lemma 8.5. Let $K$ be a field, $t$ an indeterminate, $F$ a finite Galois extension of $E=K(t)$, and $(\bar{E}, v)$ a $\mathrm{P}$-adically closed field containing $E$. Suppose $K$ is v-dense in $\bar{K}=\bar{E} \cap \tilde{K}$. Then $K$ has a nonempty v-open subset $A$ satisfying the following condition:

(3) For each $a \in A$ the $K$-specialization $t \rightarrow$ a extends to a place $\varphi$ of $F$ with residue field $F^{\prime}$ such that $\bar{K} \cap F^{\prime}$ is the residue field of $\bar{E} \cap F$.

Proof. Put $F_{0}=\bar{E} \cap F$. List the intermediate fields of $F / F_{0}$ as $F_{0}, F_{1}, \ldots, F_{m-1}, F_{m}=F$. For each $i$ between 0 and $m$ let $z_{i}$ be a primitive element for $F_{i} / E$ which is integral over $K[t]$ and let $h_{i} \in K[T, Z]$ be a polynomial satisfying $h_{i}(t, Z)=\operatorname{irr}\left(z_{i}, K(t)\right)$. Let $H=\left\{a \in \mathbb{A}^{1} \mid\right.$ $\left.\prod_{i=0}^{m} \operatorname{discr}\left(h_{i}(a, Z)\right) \neq 0\right\}$. This is a Zariski $K$-open subset of $\mathbb{A}^{1}$ and $t \in H(E)$. For each $a \in H(K)$ and for each place $\varphi$ of $F$ extending the $K$ specialization $t \rightarrow a$ the extension $F / E$ is unramified at $\varphi$ and the residue field of $F$ is a finite Galois extension of $K$. Moreover,

(4) if $E_{1}$ is an intermediate field of $F / E$ and $E_{1}^{\prime}$ is its residue field under $\varphi$, then $E_{1}^{\prime}\left(\varphi\left(z_{i}\right)\right)$ is the residue field of $E_{1}\left(z_{i}\right)=E_{1} F_{i}$ at $\varphi$, for $i=$ $0,1, \ldots, m$. In particular, $F_{i}^{\prime}=K\left(\varphi\left(z_{i}\right)\right)$ is the residue field of $F_{i}$ at $\varphi, i=0,1, \ldots, m$ [FrJ, Remark 6.1.6].

Since $F_{0}=\bar{E} \cap F$,

(5) $h_{0}(t, Z)$ has a root in $\bar{E}$, while $h_{1}(t, Z), \ldots, h_{m}(t, Z)$ have no roots in $\bar{E}$. 
Indeed if $h_{i}(t, Z)$ had a root $z_{i}^{\prime}$ in $\bar{E}$, then $z_{i}^{\prime} \in F_{0}$, so $\operatorname{deg}\left(h_{i}(t, Z)\right)=$ $\left[E\left(z_{i}^{\prime}\right): E\right] \leq\left[F_{0}: E\right]<\left[F_{i}: E\right]=\operatorname{deg}\left(h_{i}(t, Z)\right)$, which is a contradiction. By Lemma $8.4, \bar{K}$ is a P-adically closed field and $\left(\bar{K},\left.v\right|_{\bar{K}}\right)$ is an elementary submodel of $(\bar{E}, v)$. Hence, by (5) there exists $a \in H(\bar{K})$ such that

(6) $h_{0}(a, Z)$ has a root in $\bar{K}$, while $h_{1}(a, Z), \ldots, h_{m}(a, Z)$ have no root in $\bar{K}$.

By the theorem about the continuity of roots of polynomials [Jar2, Prop. 16.7 and Prop. 12.3] there is a $v$-open neighborhood $\bar{A}$ of $a$ in $\bar{K}$ such that (6) holds for each $a \in \bar{A}$. Since $K$ is $v$-dense in $\bar{K}$, there is a nonempty $v$-open set $A \subseteq K$ contained in $\bar{A}$. Without loss $A \subseteq H(K)$.

Consider $a \in A$. The $K$-specialization $t \rightarrow a$ extends to a place $\varphi$ of $F$. Let $F^{\prime}$ be its residue field and let $F_{i}^{\prime}$ be the residue field of $F_{i}$ under $\varphi$, for $i=0,1, \ldots, m$. Then $F_{0}^{\prime}, F_{1}^{\prime}, \ldots, F_{m}^{\prime}$ are intermediate fields of $F^{\prime} / F_{0}^{\prime}$. Moreover, every intermediate field of $F^{\prime} / F_{0}^{\prime}$ is of this form. Indeed, let $E_{1}$ be the decomposition field of $F / E$ at $\varphi$. Then $\left\{E_{1} F_{0}, \ldots, E_{1} F_{m}\right\}$ is the set of all intermediate fields of $F / E_{1} F_{0}$. By (4), $\left\{F_{0}^{\prime}=K\left(\varphi\left(z_{0}\right)\right), \ldots, F_{m}^{\prime}=\right.$ $\left.K\left(\varphi\left(z_{m}\right)\right)\right\}$ is the set of their residue fields. This proves our claim.

Since $\varphi\left(z_{i}\right)$ is a root of $h_{i}(a, Z)$, we may assume by (6) that $F_{0}^{\prime} \subseteq \bar{K}$ and $F_{i}^{\prime} \nsubseteq \bar{K}$ for $i=1, \ldots, m$. Consequently, $F_{0}^{\prime}=\bar{K} \cap F^{\prime}$.

\section{§9. $S_{1}$-adic hilbertianity}

The P-adic closures of a field $K$ extending a given basic P-adic field build a topological space. Given a Hilbertian field equipped with a finite set of independent "classical" P-adic fields and a set of irreducible polynomials over $K$ with algebraically independent parameters $t_{1}, \ldots, t_{r}$, we specialize the parameters to elements of $K$ and extend this specialization to a place which maps the $\mathrm{P}$-adic space over $K(\mathbf{t})$ onto the P-adic space over $K$.

Consider a P-adic field $(K, v)$ and a field extension $E$ of $K$. Let $\operatorname{Alg} \operatorname{Ext}(E, v)$ be the set of all P-adically closed algebraic extensions of $E$ whose unique $\mathrm{P}$-adic valuation or ordering extends $v$ and is of the same type as $v$. It is a topological subspace of the profinite space $\operatorname{Alg} \operatorname{Ext}(E)$ of all algebraic extensions of $E$ with the strict topology. A basic open neighborhood of a field $\bar{E} \in \operatorname{Alg} \operatorname{Ext}(E)$ is the set $\left\{E^{\prime} \in \operatorname{Alg} \operatorname{Ext}(E) \mid E^{\prime} \cap F=\bar{E} \cap F\right\}$ where $F$ is a finite Galois extension of $E$ [HJPa, Section 6]. Galois correspondence maps $\operatorname{Alg} \operatorname{Ext}(E)$ homeomorphically onto $\operatorname{Subgr}(E)$ with respect to the strict topologies. In particular, it maps $\operatorname{AlgExt}(E, v)$ onto

$$
\mathcal{G a l}(E, v)=\{\operatorname{Gal}(\bar{E}) \mid \bar{E} \in \operatorname{Alg} \operatorname{Ext}(E, v)\} .
$$


The absolute Galois group $\operatorname{Gal}(E)$ acts continuously on $\operatorname{AlgExt}(E)$ and $\operatorname{Alg} \operatorname{Ext}(E, v)$ (from the right). Let $\operatorname{AlgExt}(E, v) / \operatorname{Gal}(E)$ be the quotient space under this action. Likewise, $\operatorname{Gal}(K)$ acts on $\mathcal{G} a l(E, v)$ by conjugation from the right.

For a Galois extension $F / E$ let

$$
\begin{aligned}
\operatorname{AlgExt}(F / E, v) & =\{\bar{E} \cap F \mid \bar{E} \in \operatorname{AlgExt}(E, v)\} \\
\mathcal{G} a l(F / E, v) & =\{\operatorname{Gal}(F / \bar{E} \cap F) \mid \bar{E} \in \operatorname{AlgExt}(E, v)\} .
\end{aligned}
$$

Then $\operatorname{Gal}(F / E)$ acts on both $\operatorname{AlgExt}(F / E, v)$ and $\mathcal{G a l}(F / E, v)$ from the right. Also, the restriction to $F$ maps $\operatorname{Alg} \operatorname{Ext}(E, v)$ onto $\operatorname{Alg} \operatorname{Ext}(F / E, v)$ and $\mathcal{G} a l(E, v)$ onto $\mathcal{G} a l(F / E, v)$.

Definition 9.1. We call a P-adic field $(K, v)$ classical in each of the following cases:

(1a) $v$ is an ordering and $(K, v)$ embeds into $(\mathbb{R},<)$, where $<$ is the usual ordering of $\mathbb{R}$.

(1b) $v$ is a $p$-adic valuation and $(K, v)$ embeds into $(F, w)$ where $F$ is a finite extension of $\mathbb{Q}_{p}$ and $w$ is the extension of the $p$-adic valuation of $\mathbb{Q}_{p}$ to $F$.

In both cases the $\mathrm{P}$-adic closure $(\bar{K}, \bar{v})$ of $(K, v)$ is uniquely determined up to a $K$-isomorphism. In addition, there is a unique (up to equivalence) absolute value on $K$ which induces the $v$-adic topology on $K$. In the real case this is part of the Artin-Schreier theory [Lan, p. 455, Thm. 2.9]. In the $p$-adic case, $v$ is discrete and the statement follows from [PrR, Thm. 3.2]. Moreover, in this case $(\bar{K}, \bar{v})$ is a Henselian closure of $(K, v)$. In both cases $K$ is $v$-dense in $\bar{K}$, so Lemma 8.5 applies.

Definition 9.2. Let $S_{1}$ be a finite set of independent classical Padic orderings and valuations of $K$. Thus, the $v$-topologies of $K$ for distinct $v \in S_{1}$ are distinct. Equivalently, by the weak approximation theorem, the orderings in $S_{1}$ are distinct and the valuations in $S_{1}$ are inequivalent.

The family of all intersections of basic $v$-open sets, with $v \in S_{1}$, forms a basis for the $S_{1}$-topology of $K$. Each $S_{1}$-open set has the form $\bigcap_{v \in S_{1}} U_{v}$, where $U_{v}=\left\{x \in K \mid-\varepsilon_{v}<x-a_{v}<\varepsilon_{v}\right\}$ with $\varepsilon_{v}, a_{v} \in K$ and $\varepsilon_{v}>0$ if $v$ is an ordering $<$ and $U_{v}=\left\{x \in K \mid v\left(x-a_{v}\right)>v\left(c_{v}\right)\right\}$ with $a_{v} \in K$ and $c_{v} \in K^{\times}$if $v$ is a $p$-adic valuation. 
Definition 9.3. Let $M$ be a field and $O$ a subset of $M^{r}$. Following [JaR1, Def. 1.1], we say that $M$ is PAC over $O$ if for every absolutely irreducible variety $V$ of dimension $r \geq 0$ and for each dominating separable rational map $\varphi: V \rightarrow \mathbb{A}^{r}$ defined over $M$ there exists $\mathbf{a} \in V(M)$ such that $\varphi(\mathbf{a}) \in O$.

The next result generalizes the characterization of "PAC over $O$ " given in [JaR1, Lemma 1.3] from a subring of $M$ to an arbitrary subset $O$ of $M^{r}$.

Lemma 9.4. Let $M$ be a field and let $O$ be a subset of $M^{r}$. Then the following condition is necessary and sufficient for $M$ to be PAC over $O$ :

(2) Let $f \in M\left[T_{1}, \ldots, T_{r}, X\right]$ be an absolutely irreducible polynomial with $\frac{\partial f}{\partial X} \neq 0$ and let $0 \neq g \in M\left[T_{1}, \ldots, T_{r}\right]$. Then there exists $\mathbf{a} \in O$ and $b \in M$ such that $f(\mathbf{a}, b)=0$ and $g(\mathbf{a}) \neq 0$.

Proof. Necessity of (2) is obvious. To prove that (2) is sufficient, we consider an absolutely irreducible variety $V$ and a dominating separable rational map $\varphi: V \rightarrow \mathbb{A}^{r}$ defined over $M$. Let $\mathbf{x}$ be a generic point of $V$ over $M$ and $\mathbf{t}=\varphi(\mathbf{x})$. Then $\mathbf{t}$ is a separating transcendence basis for $M(\mathbf{x}) / M$ [Lan, p. 363]. Choose a primitive element $y$ for $M(\mathbf{x}) / M(\mathbf{t})$ which is integral over $M[\mathbf{t}]$ and let $f \in M[\mathbf{T}, Y]$ be a monic polynomial in $Y$ such that $f(\mathbf{t}, Y)=\operatorname{irr}(M(\mathbf{t}), y)$. Then $f$ is absolutely irreducible [FrJ, Cor. 10.2.2] and $\frac{\partial f}{\partial Y} \neq 0$. Denote the hypersurface in $\mathbb{A}^{r+1}$ which the equation $f(\mathbf{T}, Y)=$ 0 defines over $M$ by $W$. Let $\pi: W \rightarrow \mathbb{A}^{r}$ be the projection on the first $r$ coordinates. The map $(\mathbf{t}, y) \mapsto \mathbf{x}$ defines a birational map $\theta: W \rightarrow V$ over $M$ such that $\varphi \circ \theta=\pi$. Find a nonzero polynomial $g \in M[\mathbf{T}]$, an $M$-open subset $V_{0}$ of $V$ and an $M$-open subset $W_{0}$ of $W$ such that $\left.\varphi\right|_{V_{0}}: V_{0} \rightarrow \mathbb{A}^{r}$ is a morphism, $\left.\theta\right|_{W_{0}}: W_{0} \rightarrow V_{0}$ is an isomorphism, and $W_{0}=\pi^{-1}\left(\mathbb{A}^{r} \backslash V(g)\right)$. By (2) there exists $\mathbf{a} \in O$ and $b \in M$ such that $f(\mathbf{a}, b)=0$ and $g(\mathbf{a}) \neq 0$. Let $\mathbf{c}=\theta(\mathbf{a}, b)$. Then $(\mathbf{a}, b) \in W_{0}(M), \mathbf{c} \in V(M)$, and $\varphi(\mathbf{c})=\mathbf{a} \in O$. Consequently, $M$ is PAC over $O$.

The following result (except for Condition (3a), which is new) is an analog of [FHV, Lemma 3].

Lemma 9.5. Let $K$ be a Hilbertian field [FrJ, Sec. 12.1], $S_{1}$ a finite set of independent classical $\mathrm{P}$-adic orderings and valuations of $K$, and $K_{0}$ a separable algebraic extension of $K$. Let $L$ be a finite Galois extension of $K$, $t$ an indeterminate, and $F$ a finite Galois extension of $K(t)$ which is regular over $L$, and $F_{0}$ an extension of $K(t)$ in $F$. Set $L_{0}=K_{0} \cap L$. Suppose 
(3a) $K_{0}$ is PAC over each subset $H \cap A$, where $H$ is a Hilbert subset of $K^{r}$ and $A$ is a nonempty $S_{1}$-open subset of $K^{r}$,

(3b) $F_{0} \cap L=L_{0}$, and $F_{0} L=F$.

Then there exists an epimorphism $\gamma: \operatorname{Gal}(K) \rightarrow \operatorname{Gal}(F / K(t))$ such that $\operatorname{res}_{F / L} \circ \gamma=\operatorname{res}_{\tilde{K} / L}, \gamma\left(\operatorname{Gal}\left(K_{0}\right)\right)=\operatorname{Gal}\left(F / F_{0}\right)$, and for each $v \in S_{1}$ we have $\gamma(\mathcal{G a l}(K, v))=\mathcal{G} a l(F / K(t), v)$.

Proof. By abuse of notation, we abbreviate a place of fields $\psi: M \rightarrow$ $N \cup\{\infty\}$ to $\psi: M \rightarrow N$ and write $\psi(M)$ for the residue field of $M$ under $\psi$.

PART A: Hilbertianity. There is a Hilbert subset $H$ of $K$ with the following property: For each $a \in H$ each extension of the specialization $t \mapsto$ $a$ to an $L$-place $\varphi_{a}: F \rightarrow \tilde{K}$ with residue field $F_{a}$ induces an isomorphism $\gamma_{a}: \operatorname{Gal}\left(F_{a} / K\right) \rightarrow \operatorname{Gal}(F / K(t))$ such that $\varphi_{a}\left(\gamma_{a}(\sigma)(x)\right)=\sigma\left(\varphi_{a}(x)\right)$ for each $x \in F$ with $\varphi_{a}(x) \neq \infty$ and each $\sigma \in \operatorname{Gal}\left(F_{a} / K\right)$; in particular $\operatorname{res}_{F / L} \circ \gamma_{a}=$ $\operatorname{res}_{F_{a} / L}$ [FrJ, Lemma 13.1.1]. Put $\gamma=\gamma_{a} \circ \operatorname{res}_{\tilde{K} / F_{a}}$. Then $\operatorname{res}_{F / L} \circ \gamma=$ $\operatorname{res}_{\tilde{K} / L}$. Thus, it suffices to choose $a \in H$ such that $\gamma_{a}\left(\operatorname{Gal}\left(F_{a} / F_{a} \cap K_{0}\right)\right)=$ $\operatorname{Gal}\left(F / F_{0}\right)$ and $\gamma_{a}\left(\mathcal{G a l}\left(F_{a} / K, v\right)\right)=\mathcal{G a l}(F / K(t), v)$ for each $v \in S_{1}$.

Let $E$ be a field between $K(t)$ and $F$. Denote the residue field of $E$ under $\varphi_{a}$ by $E_{a}$. Then $\gamma_{a}\left(\operatorname{Gal}\left(F_{a} / E_{a}\right)\right)=\operatorname{Gal}(F / E)$. Therefore the map $E \mapsto E_{a}$ is a bijection between the lattices of intermediate fields of $F / K(t)$ and of $F_{a} / K$. Then it suffices to choose $a \in H$ such that

(4a) $F_{0, a}=K_{0} \cap F_{a}$, and

(4b) $E \in \operatorname{Alg} \operatorname{Ext}(F / K(t), v) \Leftrightarrow E_{a} \in \operatorname{Alg} \operatorname{Ext}\left(F_{a} / K, v\right)$ for each $v \in S_{1}$.

Indeed, in that case $\gamma\left(\operatorname{Gal}\left(K_{0}\right)\right)=\gamma_{a}\left(\operatorname{Gal}\left(F_{a} / K_{0} \cap F_{a}\right)\right)=\gamma_{a}\left(\operatorname{Gal}\left(\left(F_{a} /\right.\right.\right.$ $\left.\left.F_{0, a}\right)\right)=\operatorname{Gal}\left(F / F_{0}\right)$ and $\gamma(\mathcal{G a l}(K, v))=\gamma_{a}\left(\mathcal{G} a l\left(F_{a} / K, v\right)\right)=\operatorname{Gal}(F / K(t), v)$ for each $v \in S_{1}$.

PART B: Lifting. Suppose $E_{a} \in \operatorname{AlgExt}\left(F_{a} / K, v\right)$. Then there is a P-adic closure $(\bar{K}, \bar{v})$ of $(K, v)$ such that $\bar{K} \cap F_{a}=E_{a}$. By Lemma 8.3, there is a P-adic closure $(\bar{E}, \bar{w})$ of $E$ which extends $(\bar{K}, \bar{v})$ such that the restriction $E \rightarrow E_{a}$ of $\varphi_{a}$ to $E$ extends to a place $\bar{\varphi}_{a}: \bar{E} \rightarrow \bar{K}$. Then

$$
E_{a}=\varphi_{a}(E) \subseteq \varphi_{a}(\bar{E} \cap F) \subseteq \varphi_{a}(\bar{E}) \cap \varphi_{a}(F)=\bar{K} \cap F_{a}=E_{a}
$$

Therefore, $\varphi_{a}(E)=\varphi_{a}(\bar{E} \cap F)$. By the bijection in Part A, $E=\bar{E} \cap F$. In addition, $\bar{E} \in \operatorname{Alg} \operatorname{Ext}(K(t), v)$, so $E \in \operatorname{Alg} \operatorname{Ext}(F / K(t), v)$. 
PART C: Open neighborhoods. Suppose $E \in \operatorname{AlgExt}(F / K(t), v)$. Then, there is a P-adic closure $\bar{E} \in \operatorname{Alg} \operatorname{Ext}(K(t), v)$ of $E$ such that $\bar{E} \cap F=$ $E$. Extend $v$ to a P-adic ordering or valuation of $\bar{E}$ and let $\bar{K}=\bar{E} \cap \tilde{K}$. Then $\bar{K} \in \operatorname{Alg} \operatorname{Ext}(K, v)$. By Definition $9.1, K$ is $v$-dense in $\bar{K}$.

By Lemma 8.5, $K$ has a $v$-open subset $A_{v}$ such that for each $a \in A_{v}$ the $K$-specialization $t \rightarrow a$ extends to a place $\varphi_{a}$ of $F$ with residue field $F_{a}$ such that $\bar{K} \cap F_{a}$ is the residue field $E_{a}$ of $E$. Hence, if $a \in A_{v}$, then $E_{a} \in \operatorname{Alg} \operatorname{Ext}\left(F_{a} / K, v\right)$.

PART D: Conclusion. By assumption, the orderings and valuations in $S$ are classical and independent. Hence, by [Gey, Lemma 3.4], $H \cap$ $\bigcap_{v \in S} A_{v} \neq \emptyset$. By assumption, $F_{0} \cap L=L_{0}, F_{0} L=F$, and $F / L$ is regular. Choose a primitive element $x$ for $F_{0} / L_{0}(t)$ which is integral over $L_{0}[t]$ and let $f \in L_{0}[T, X]$ be a monic polynomial in $X$ such that $f(t, X)=\operatorname{irr}\left(x, L_{0}(t)\right)$. Then $f \in K_{0}[T, X]$ and $f$ is absolutely irreducible [FrJ, Cor. 10.2.2(b)]. Moreover, $\operatorname{discr}(f(t, X)) \neq 0$, so we may make $H$ smaller, if necessary, such that for each $a \in H$ each extension of the specialization $t \rightarrow a$ to an $L$-place $\varphi_{a}: F \rightarrow \tilde{K}$, we have $\operatorname{discr}(f(a, X)) \neq 0$, hence $\varphi_{a}\left(F_{0}\right)=L_{0}\left(\varphi_{a}(x)\right)$.

By (3a), we may choose $a \in H \cap \bigcap_{v \in S} A_{v}$ and $b \in K_{0}$ such that $f(a, b)=$ 0 . Now we extend the specialization $t \rightarrow a$ to an $L$-place $\varphi_{a}: F \rightarrow \tilde{K}$ such that $\varphi_{a}(x)=b$. Let $F_{a}=\varphi_{a}(F)$ and $F_{0, a}=\varphi_{a}\left(F_{0}\right)$. Then $F_{0, a}=L_{0}(b) \subseteq$ $K_{0}$, so $\left[F: F_{0}\right] \geq\left[F_{a}: F_{0, a}\right] \geq\left[F_{a}: K_{0} \cap F_{a}\right] \geq\left[L: L_{0}\right]=\left[F: F_{0}\right]$. It follows that all of the latter inequalities are in fact equalities and $K_{0} \cap F_{a}=F_{0, a}$. By Part A, this implies that $\gamma\left(\operatorname{Gal}\left(K_{0}\right)\right)=\operatorname{Gal}\left(F / F_{0}\right)$, so (4a) holds. Finally, (4b) follows from Parts B and $C$.

\section{$\S 10$. Totally $S_{1}$-adic extensions}

Starting from a countable Hilbertian field $K$, a set $S_{1}$ of classical P-adic orderings and valuations of $K$, and a distinguished algebraic extension $K_{0}$ of $K$, we consider the maximal totally $S_{1}$-adic extension $K_{\text {tot, } S_{1}}$ of $K$ and the field

$$
M=K_{0} \cap K_{\text {tot, } S_{1}}=K_{0} \cap \bigcap_{v \in S_{1}} \bigcap_{\sigma \in \operatorname{Gal}(K)} K_{v}^{\sigma}
$$

and note that $M \subseteq K_{v}$ for each $v \in S_{1}$. Proposition 10.5 gives a weak solution to an embedding problem over $M$ with local data. The proof of that proposition reduces the problem over $M$ to an embedding problem over a finite extension $K^{\prime}$ of $K$ and then uses Lemma 9.5 to solve the reduced problem. 
SETuP 10.1. For the rest of this section and the next one we fix a countable Hilbertian field $K$ of characteristic 0 , an algebraic closure $\tilde{K}$ of $K$, a field extension $K_{0}$ of $K$ in $\tilde{K}$, and a finite set $S_{1}$ of independent classical P-adic orderings and valuations of $K$; in particular $0,1 \notin S_{1}$. Set $S_{0}=\{0\}$ and $S=S_{0} \cup S_{1}$.

For an extension $E$ of $K$ let

$$
\begin{aligned}
\operatorname{Alg} \operatorname{Ext}\left(E, S_{1}\right) & =\bigcup_{v \in S_{1}} \operatorname{AlgExt}(E, v) \text { and } \\
\mathcal{G} a l\left(E, S_{1}\right) & =\bigcup_{v \in S_{1}} \mathcal{G} a l(E, v) .
\end{aligned}
$$

For a Galois extension $F$ of $E$ let

$$
\begin{aligned}
\operatorname{Alg} \operatorname{Ext}\left(F / E, S_{1}\right) & =\bigcup_{v \in S_{1}} \operatorname{AlgExt}(F / E, v) \text { and } \\
\mathcal{G} a l\left(F / E, S_{1}\right) & =\bigcup_{v \in S_{1}} \mathcal{G} a l(F / E, v)
\end{aligned}
$$

The maximal totally $S_{1}$-adic extension of $K$ is the intersection of all $\bar{K} \in \operatorname{Alg} \operatorname{Ext}\left(K, S_{1}\right)$. We denote it by $K_{\text {tot, } S_{1}}$. It is a Galois extension of $K$, because each $\operatorname{Alg} \operatorname{Ext}(K, v)$ is closed under the conjugation by elements of $\operatorname{Gal}(K)$. For each $v \in S_{1}$ we choose a real closure of $K$ at $v$ if $v$ is an ordering or a Henselian closure $K_{v}$ of $K$ at $v$ if $v$ is a valuation.

We also set $M=K_{0} \cap K_{\text {tot }, S_{1}}, \mathcal{G a l}\left(M, S_{0}\right)=\left\{\operatorname{Gal}\left(K_{0}\right)^{\tau} \mid \tau \in \operatorname{Gal}(M)\right\}$, $\mathcal{G} a l(M, S)=\mathcal{G a l}\left(M, S_{0}\right) \cup \mathcal{G a l}\left(M, S_{1}\right)$, and make the following assumptions on $K_{0}$ and $M$ :

(1a) $K_{0}$ is PAC over each set $H \cap A$, where $H$ is a Hilbertian subset of $K^{r}$ and $A$ is a nonempty open $S_{1}$-adic subset of $K^{r}$.

(1b) $\mathrm{Gal}\left(K_{0}\right)$ is a finitely generated free profinite group.

(1c) $M$ is $\mathrm{P} S_{1} \mathrm{C}$. This means that every absolutely irreducible variety $V$ which is defined over $M$ and has a simple $K_{v}$-rational point for each $v \in S_{1}$ has an $M$-rational point.

(1d) $[M: K]=\infty$.

Corollary 10.2. The field $M$ is ample. That is, every absolutely irreducible curve $C$ defined over $M$ with a simple $M$-rational point has infinitely many $M$-rational points. 
Proof. Let $\mathbf{p}$ be a simple $M$-rational point on $C$. Consider $\mathbf{q}_{1}, \ldots, \mathbf{q}_{n} \in$ $C(M)$. Then $C^{\prime}=C \backslash\left\{\mathbf{q}_{1}, \ldots, \mathbf{q}_{n}\right\}$ is also an absolutely irreducible curve defined over $M$. Let now $v \in S_{1}$. Then $\mathbf{p} \in C_{\text {simp }}\left(K_{v}\right)$. Hence, $C\left(K_{v}\right)$ is infinite, because $K_{v}$ is either real closed or Henselian [GPR, Thm. 9.2]. Hence, $C_{\text {simp }}^{\prime}\left(K_{v}\right) \neq \emptyset$. Applying the $\mathrm{P} S_{1} \mathrm{C}$ property of $M$ to $C^{\prime}$, we conclude that $C(M)$ has an additional point $\mathbf{q}_{n+1}$.

LEMMA 10.3. The following statements hold for $M$ and for each $v \in$ $S_{1}$ :

(a) $\operatorname{Alg} \operatorname{Ext}(M, v)=\operatorname{Alg} \operatorname{Ext}(K, v)=\left\{K_{v}^{\sigma} \mid \sigma \in \operatorname{Gal}(K)\right\}$.

(b) $K_{v}^{\sigma}=K_{v}$ if and only if $\sigma \in \operatorname{Gal}\left(K_{v}\right)$.

(c) $K_{0} \notin \operatorname{Alg} \operatorname{Ext}\left(M, S_{1}\right)$ and the sets $\operatorname{Alg} \operatorname{Ext}(M, v), v \in S_{1}$, are disjoint. Moreover, there are no inclusions among distinct fields belonging to $\operatorname{Alg} \operatorname{Ext}\left(M, S_{1}\right)$.

(d) Let $E$ be a field extension of $K$. Then $\operatorname{AlgExt}(E, v)$ is closed in $\operatorname{Alg} \operatorname{Ext}(E)$.

(e) The topological space $\operatorname{Alg} \operatorname{Ext}(M, v) / \operatorname{Gal}(M)$ has no isolated points.

(f) Every finite split embedding problem over $M$ is regularly solvable over $M(t)$. That is, let $N / M$ be a finite Galois extension, $B$ a finite group, $\alpha: B \rightarrow \operatorname{Gal}(N / M)$ an epimorphism admitting a group theoretic section, $\lambda: \operatorname{Gal}(M(t)) \rightarrow \operatorname{Gal}(M)$ the restriction map, and $\varphi: \operatorname{Gal}(M(t)) \rightarrow \operatorname{Gal}(N / M)$ an epimorphism. Then there exists an epimorphism $\delta: \operatorname{Gal}(M(t)) \rightarrow \operatorname{Gal}(N / M)$ such that $\alpha \circ \delta=\varphi$ and $\lambda(\operatorname{Ker}(\delta))=\operatorname{Ker}(\varphi)$. Equivalently (Remark 7.6), the fixed field $F$ of $\operatorname{Ker}(\delta)$ in $\widetilde{M(t)}$ is regular over $N$.

Proof of (a). As indicated in Definition 9.1, the set $\operatorname{AlgExt}(K, v)$ of the P-adic closures of $(K, v)$ coincides with the set of Henselian closures of $K$ at $v$ in $\tilde{K}$. Thus $\operatorname{Alg} \operatorname{Ext}(K, v)=\left\{K_{v}^{\sigma} \mid \sigma \in \operatorname{Gal}(K)\right\}$. By definition, $\operatorname{Alg} \operatorname{Ext}(M, v)=\operatorname{Alg} \operatorname{Ext}(K, v)$.

Proof of (b). Suppose $K_{v}^{\sigma}=K_{v}$. Then $\left.\sigma\right|_{K_{v}}$ belongs to $\operatorname{Aut}\left(K_{v} / K\right)$. By [Lan, p. 455, Thm. 2.9] for real closed fields and [Jar2, Prop. 14.5] for Henselian closures, $\operatorname{Aut}\left(K_{v} / K\right)$ is trivial, so $\sigma \in \operatorname{Gal}\left(K_{v}\right)$.

Proof of (c). By (1a), $K_{0}$ is PAC. As such, $K_{0}$ is neither real closed [FrJ, Thm. 11.5.1] nor does $K_{0}$ have a valuation with a finite residue field [FrJ, p. 217, Exercise 7(b)]. Thus, $K_{0} \notin \operatorname{AlgExt}\left(K, S_{1}\right)$. 
Next note that, by Lemma 8.4, no field in $\operatorname{Alg} \operatorname{Ext}\left(K, S_{1}\right)$ is algebraically closed. Consider distinct fields $K^{\prime}, K^{\prime \prime} \in \operatorname{Alg} \operatorname{Ext}\left(K, S_{1}\right)$. Assume that $K^{\prime} \subset$ $K^{\prime \prime}$. Since the absolute Galois group of a real closed field is of order 2 while the absolute Galois group of a $p$-adically closed field is torsion free [HJPb, Lemma 8.3], neither $K^{\prime}$ nor $K^{\prime \prime}$ are real closed.

If $K^{\prime} \in \operatorname{AlgExt}\left(K, v^{\prime}\right)$ and $K^{\prime \prime} \in \operatorname{AlgExt}\left(K, v^{\prime \prime}\right)$ for some $v^{\prime}, v^{\prime \prime} \in S_{1}$ with $v^{\prime} \neq v^{\prime \prime}$, then $K^{\prime \prime}$ is Henselian with respect to $v^{\prime \prime}$ and to an extension of $v^{\prime}$. Since, $v^{\prime}$ and $v^{\prime \prime}$ are independent and $K^{\prime \prime}$ is not separably closed (Lemma 8.4(c)), this contradicts [Jar2, Lemma 13.2].

If $K^{\prime}, K^{\prime \prime} \in \operatorname{Alg} \operatorname{Ext}(K, v)$ for some $v \in S_{1}$, then $K^{\prime}$ and $K^{\prime \prime}$ are conjugate over $K$, so $K^{\prime \prime}$ cannot properly contain $K^{\prime}$ [FrJ, Lemma 20.6.2].

Proof of (d). Let $\bar{E} \in \operatorname{AlgExt}(E)$ and put $\bar{K}=\bar{E} \cap \tilde{K}$. If $\bar{E} \in$ $\operatorname{Alg} \operatorname{Ext}(E, v)$, then, by Lemma 8.4, $\bar{K} \in \operatorname{Alg} \operatorname{Ext}(K, v)$ and $\bar{E} \equiv \bar{K}$. By (a), $\bar{K}$ is isomorphic to $K_{v}$. Hence, $\bar{E} \equiv K_{v}$. Conversely, if $\bar{K} \in \operatorname{AlgExt}(K, v)$ and $\bar{E} \equiv K_{v}$, then $\bar{E}$ is P-adically closed of the same type as $K_{v}$. In the $p$-adic case this follows from [HJPb, Proposition 8.2(h)]. In the real case $\bar{E}$ is real closed and our conclusion follows. Therefore, $\bar{E} \in \operatorname{Alg} \operatorname{Ext}(E, v)$. It follows that $\operatorname{Alg} \operatorname{Ext}(E, v)$ is the intersection of $A_{1}=\{\bar{E} \in \operatorname{AlgExt}(E) \mid$ $\bar{E} \cap \tilde{K} \in \operatorname{Alg} \operatorname{Ext}(K, v)\}$ and $A_{2}=\left\{\bar{E} \in \operatorname{Alg} \operatorname{Ext}(E) \mid \bar{E} \equiv K_{v}\right\}$.

By (a), $\operatorname{Alg} \operatorname{Ext}(K, v)$ is closed in $\operatorname{Alg} \operatorname{Ext}(K)$. Since the restriction $\operatorname{Alg} \operatorname{Ext}(E) \rightarrow \operatorname{Alg} \operatorname{Ext}(K)$ is continuous, $A_{1}$ is closed in $\operatorname{AlgExt}(E)$. By [HJPb, Lemma 10.1], also $A_{2}$ is closed in $\operatorname{AlgExt}(E)$. Consequently, $\operatorname{Alg} \operatorname{Ext}(E, v)$ is closed in $\operatorname{Alg} \operatorname{Ext}(E)$.

Proof of (e). The map $\operatorname{Gal}(K) \rightarrow \operatorname{AlgExt}(K)$ given by $\sigma \mapsto K_{v}^{\sigma}$ is a continuous map of profinite spaces. By (a), its image is $\operatorname{Alg} \operatorname{Ext}(M, v)$. By (b), $K_{v}^{\sigma_{1}}=K_{v}^{\sigma_{2}}$ with $\sigma_{1}, \sigma_{2} \in \operatorname{Gal}(K)$ if and only if $\sigma_{2} \in \operatorname{Gal}\left(K_{v}\right) \sigma_{1}$. Therefore $\operatorname{Gal}(K) \rightarrow \operatorname{AlgExt}(K)$ induces a homeomorphism $\operatorname{Gal}\left(K_{v}\right) \backslash \operatorname{Gal}(K) \rightarrow$ $\operatorname{Alg} \operatorname{Ext}(M, v)$. This map is compatible with the action of $\operatorname{Gal}(M)$ on both spaces (on $\operatorname{Gal}\left(K_{v}\right) \backslash \operatorname{Gal}(K)$ by multiplication from the right) and hence induces a homeomorphism of quotient spaces $\operatorname{Gal}\left(K_{v}\right) \backslash \operatorname{Gal}(K) / \operatorname{Gal}(M) \rightarrow$ $\operatorname{Alg} \operatorname{Ext}(M, v) / \operatorname{Gal}(M)$. Thus, by Lemma 2.2, it suffices to show that $\operatorname{Gal}\left(K_{v}^{\sigma}\right) \operatorname{Gal}(M)$ is an open subset of $\operatorname{Gal}(K)$ for no $\sigma \in \operatorname{Gal}(K)$. But $M \subseteq K_{v}^{\sigma}$ and $[M: K]=\infty$ (Condition (1d)), hence $\operatorname{Gal}\left(K_{v}^{\sigma}\right) \operatorname{Gal}(M)=$ $\operatorname{Gal}(M)$ is not open.

Proof of (f). By (1c), $M$ is $\mathrm{P} S_{1}$ C. Hence, by Corollary 10.2, $M$ is ample. Therefore, every finite split embedding problem over $M$ is regularly solvable over $M(t)$ ([Pop4, Main Theorem A] or [HaJ3, Thm. C]). 
We rewrite the results of Lemma 10.3 in group theoretic terms. To this end we add the following notation to Setup 10.1:

$$
\begin{aligned}
\mathcal{C}_{1} & =\left\{\operatorname{Gal}\left(K_{v}\right) \mid v \in S_{1}\right\} \\
\mathcal{G} a l(M, 0) & =\left\{\operatorname{Gal}\left(K_{0}\right)^{\sigma} \mid \sigma \in \operatorname{Gal}(M)\right\} \\
\mathcal{G} a l(M, v) & =\left\{\operatorname{Gal}\left(K_{v}\right)^{\sigma} \mid \sigma \in \operatorname{Gal}(K)\right\}, \quad v \in S_{1} \\
\mathcal{G} a l\left(M, S_{1}\right) & =\bigcup_{v \in S_{1}} \mathcal{G a l}(M, v) \\
\mathcal{G} a l(M, S) & =\bigcup_{v \in S} \mathcal{G a l}(M, v) \\
\operatorname{Gal}(M, S) & =(\operatorname{Gal}(M), \mathcal{G} a l(M, v))_{v \in S}
\end{aligned}
$$

Proposition 10.4. In the above notation, the following holds:

(a) Each group in $\mathcal{C}_{1}$ has a big quotient with respect to $\mathcal{C}_{1}$.

(b) There are no inclusions between distinct groups in $\mathcal{G} a l\left(M, S_{1}\right)$.

(c) $\mathcal{G a l}(M, S)=\bigcup_{v \in S} \mathcal{G a l}(M, v)$ and for each $v \in S, \mathcal{G} a l(M, v)$ is openclosed in $\mathcal{G a l}(M, S)$ and each group in $\mathcal{G} a l(M, v)$ is isomorphic to $\operatorname{Gal}\left(K_{v}\right)$.

(d) $\operatorname{Gal}(M)$ is $\mathcal{G} a l\left(M, S_{1}\right)$-projective.

(e) For each $v \in S_{1}$ the space $\mathcal{G} a l(M, v) / \operatorname{Gal}(M)$ has no isolated points.

(f) $\operatorname{Gal}(M, S)$ is a self-generated group pile.

Proof of (a). By [HJPb, Prop. 8.2(j), (m) and Remark 8.4], each $\operatorname{Gal}\left(K_{v}\right)$ with $v \in S$ is isomorphic to $\operatorname{Gal}(\mathbb{R})$ or to $\operatorname{Gal}(\mathbb{F})$, where $\mathbb{F}$ is a finite extension of $\mathbb{Q}_{p}$ for some prime number $p$ (note that our definition of $\mathrm{P}$-adically closed fields has been extended to include $\mathbb{R}$ ). Hence, by $[\mathrm{HJPb}$, Lemma 9.4], each group in $\mathcal{C}_{1}$ has a big quotient with respect to $\mathcal{C}_{1}$.

Proof of (b). By Lemma 10.3(c), there are no inclusions among distinct elements of $\operatorname{Alg} \operatorname{Ext}\left(M, S_{1}\right)$, so there are no inclusions among distinct elements of $\mathcal{G a l}\left(M, S_{1}\right)$.

Proof of (c). By definition, $\mathcal{G} a l(M, 0)$ is a closed $\operatorname{Gal}(M)$-class. For each $v \in S_{1}, \operatorname{Alg} \operatorname{Ext}(M, v)$ is closed in $\operatorname{AlgExt}(M)$ (Lemma 10.3(d)), so $\mathcal{G} a l(M, v)$ is closed in $\operatorname{Gal}(M)$ and in particular in $\mathcal{G} a l(M, S)$. By 10.3(c), $\operatorname{Gal}\left(K_{0}\right) \notin \mathcal{G} a l\left(M, S_{1}\right)$, so $\mathcal{G} a l(M, 0)$ is disjoint from $\mathcal{G} a l\left(M, S_{1}\right)$. By (b), the sets $\mathcal{G a l}(M, v), v \in S_{1}$, are disjoint. Therefore, $\mathcal{G} a l(M, S)=\bigcup_{v \in S} \mathcal{G} a l(M, v)$ 
is a partition into open closed sets. Finally, each group in $\mathcal{G} a l(M, 0)$ is by definition isomorphic to $\operatorname{Gal}\left(K_{0}\right)$, and for $v \in S_{1}$ each group in $\operatorname{Gal}(M, v)$ is isomorphic to $\operatorname{Gal}\left(K_{v}\right)$ (Lemma 10.3(a)).

Proof of (d). By Assumption (1c), $M$ is P $S_{1}$ C. By (c) and the second paragraph of Section $2, \mathcal{G} a l\left(M, S_{1}\right)$ is étale compact. It follows from [HJPb, Prop. 4.1] that $\operatorname{Gal}(M)$ is $\mathcal{G} a l\left(M, S_{1}\right)$-projective.

Proof of (e). Let $v \in S_{1}$. By Lemma 10.3(e), $\operatorname{AlgExt}(M, v) / \operatorname{Gal}(M)$ has no isolated points. Hence, $\operatorname{Gal}(M, v) / \operatorname{Gal}(M)$ has no isolated points.

Proof of (f). By definition, $\mathcal{G} a l\left(M, S_{0}\right)$ is a $\operatorname{Gal}(M)$-class in Subgr $(\operatorname{Gal}(M))$. By $(\mathrm{c}), \mathcal{G} a l(M, v)$ is a closed $\operatorname{Gal}(M)$-domain in $\operatorname{Subgr}(\operatorname{Gal}(M))$, $v \in S_{1}$. Hence, $\operatorname{Gal}(M, S)$ is a group pile. By Lemma $10.3(\mathrm{~b}), \operatorname{Gal}(M)=$ $\left\langle\operatorname{Gal}\left(K_{0}\right), \mathcal{G a l}\left(M, S_{1}\right)\right\rangle$. Consequently, $\mathbf{G a l}(M)$ is self-generated.

Proposition 10.5. In the above notation, let $N$ be a finite Galois extension of $M, t$ an indeterminate, $P$ a finite Galois extension of $M(t)$ which is regular over $N$, and $P_{0}$ a subfield of $P$ which contains $M(t)$. Set $N_{0}=K_{0} \cap N$ and suppose that $P_{0} \cap N=N_{0}$ and $P_{0} N=P$. Then there exists a homomorphism $\gamma: \operatorname{Gal}(M) \rightarrow \operatorname{Gal}(P / M(t))$ such that $\operatorname{res}_{P / N}$ o $\gamma=$ $\operatorname{res}_{\tilde{M} / N}, \gamma\left(\operatorname{Gal}\left(K_{0}\right)\right)=\operatorname{Gal}\left(P / P_{0}\right)$, and $\gamma(\mathcal{G a l}(M, v))=\mathcal{G a l}(P / M(t), v)$ for each $v \in S_{1}$.

Proof. The proof naturally breaks up into three parts.

PART A: Replacing $K$ by a finite extension. Let $K^{\prime}$ be a finite extension of $K$ contained in $M$. For each $v \in S_{1}$ we have $\mathcal{G} a l(P / M(t), v)=$ $\bigcup_{v^{\prime} \in \operatorname{Val}\left(K^{\prime}, v\right)} \mathcal{G} a l\left(P / M(t), v^{\prime}\right)$ and $\mathcal{G a l}(N / M, v)=\bigcup_{v^{\prime} \in \operatorname{Val}\left(K^{\prime}, v\right)} \mathcal{G a l}\left(N / M, v^{\prime}\right)$, where $\operatorname{Val}\left(K^{\prime}, v\right)$ is the set of all extensions of $v$ to $K^{\prime}$ of the same type as $v$. Therefore we may replace $K$ by $K^{\prime}$ and $S_{1}$ by $S_{1}^{\prime}=\bigcup_{v \in S} \operatorname{Val}\left(K^{\prime}, v\right)$. Now we choose a suitable $K^{\prime}$.

Let $c \in N$ with $N=M(c)$ and let $f(X)=\operatorname{irr}(c, M) \in M[X]$. Choose a finite extension $K^{\prime}$ of $K$ contained in $M$, such that $f \in K^{\prime}[X]$ and $f$ splits over $K^{\prime}(c)$ into linear factors. Put $L=K^{\prime}(c)$ and $L_{0}=N_{0} \cap L$. Then $L / K^{\prime}$ is a finite Galois extension such that $M L=N, M \cap L=K^{\prime}$, and $L_{0}=K_{0} \cap N \cap L=K_{0} \cap L$. In addition, $N_{0} L=N_{0}(c)=N$, hence $\left[N: N_{0}\right]=\left[L: L_{0}\right]$.

Now choose $z \in P$ integral over $M[t]$ such that $P=M(t, z)$ and let $g(t, X)=\operatorname{irr}(z, M(t)) \in M[t, X]$. Let $p \in K[T, X]$ and $q \in K[T]$ such that $q \neq 0$ and $c=\frac{p(t, z)}{q(t)}$. Put $F=K^{\prime}(t, z)$. If $K^{\prime}$ is sufficiently large, then it 
contains the coefficients of $g, p$, and $q$, and $g(t, X)$ splits over $F$ into linear factors. In this case $F$ is a finite Galois extension of $K^{\prime}(t)$ containing $L$ such that $M(t) F=P$ and $M(t) \cap F=K^{\prime}(t)$.

By the first paragraph of this part we may assume that $K^{\prime}=K$. This gives a diagram of fields

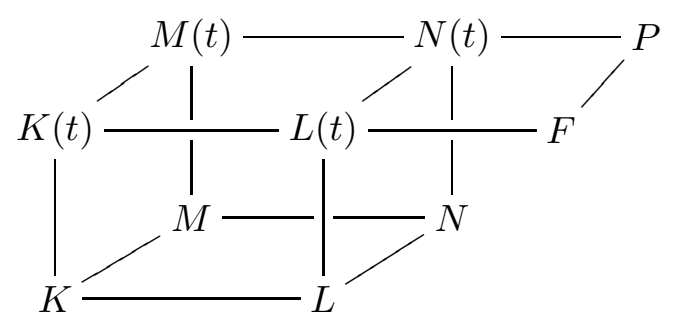

in which $M L=N, M \cap L=K, M(t) F=P$, and $M(t) \cap F=K(t)$. Thus, $M(t)$ and $F$ are linearly disjoint over $K(t)$. Since $M(t) L(t)=N(t)$, it follows that $N(t)$ is linearly disjoint from $F$ over $L(t)$. Hence, $N$ is linearly disjoint from $F$ over $L$. Since $N F=P$ and $P$ is linearly disjoint from $\tilde{K}$ over $N$ (because $P / N$ is regular), $F$ is linearly disjoint from $\tilde{K}$ over $L$. This means that $F / L$ is regular.

Next let $F_{0}=P_{0} \cap F$ and observe that $F_{0} \cap L=P_{0} \cap F \cap L=P_{0} \cap N \cap L=$ $N_{0} \cap L=L_{0}$. Since $\left[P: P_{0}\right]=\left[N: N_{0}\right]=\left[L: L_{0}\right]$ and $\left[P: P_{0}\right] \geq\left[F_{0}: F\right] \geq$ $\left[L: L_{0}\right]$, we have $\left[F: F_{0}\right]=\left[L: L_{0}\right]$, so $F_{0} L=F$. This gives commutative diagrams of fields and of Galois groups in which each of the restriction maps is an isomorphism:
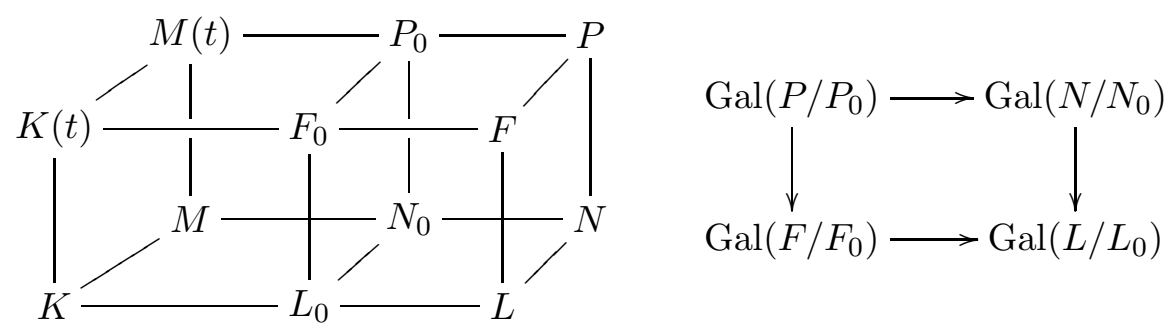

In addition, we have the following commutative diagrams of restrictions of Galois groups and sets of subgroups for each $v \in S_{1}$.

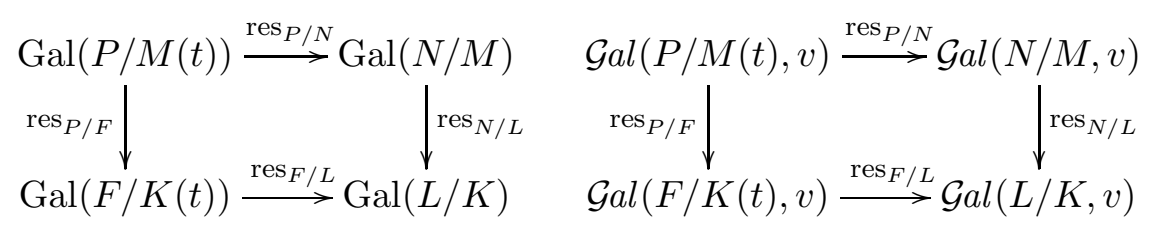


PART B: We prove that the vertical maps in diagram (2) are bijections. By Part A, the vertical maps in the diagram on the left are isomorphisms. The vertical maps in the diagram on the right are induced by them, hence they are injective. We show they are surjective. Every field in $\operatorname{Alg} \operatorname{Ext}(F / K(t), v)$ has the form $\bar{E} \cap F$ for some $\bar{E} \in \operatorname{Alg} \operatorname{Ext}(K(t), v)$. Then, $\bar{E} \cap \tilde{K} \in \operatorname{Alg} \operatorname{Ext}(K, v)$. By assumption, $M \subseteq \bar{E} \cap \tilde{K}$ and $K(t) \subseteq \bar{E}$, so $M(t) \subseteq \bar{E}$. Therefore $\bar{E} \cap P \in \operatorname{Alg} \operatorname{Ext}(P / M(t), v)$ and $(\bar{E} \cap P) \cap F=\bar{E} \cap F$, as claimed. Similarly $\operatorname{res}_{N / L}$ is surjective.

PART C: Homomorphism. By Lemma 9.5, there exists an epimorphism $\gamma_{1}: \operatorname{Gal}(K) \rightarrow \operatorname{Gal}(F / K(t))$ such that $\operatorname{res}_{F / L} \circ \gamma_{1}=\operatorname{res}_{\tilde{K} / L}, \gamma_{1}\left(\operatorname{Gal}\left(K_{0}\right)\right)=$ $\operatorname{Gal}\left(F / F_{0}\right)$, and $\gamma_{1}\left((\mathcal{G a l}(K, v))=\mathcal{G} a l(F / K(t), v)\right.$ for each $v \in S_{1}$.

Let $\gamma_{2}=\operatorname{res}_{P / F}^{-1} \circ \gamma_{1}: \operatorname{Gal}(K) \rightarrow \operatorname{Gal}(P / M(t))$. Set $\gamma$ to be the restriction of $\gamma_{2}$ to $\operatorname{Gal}(M)$. By the commutativity of (2) and by Claim B we have $\operatorname{res}_{P / N} \circ \gamma=\operatorname{res}_{\tilde{M} / N}, \gamma\left(\operatorname{Gal}\left(K_{0}\right)\right)=\operatorname{Gal}\left(P / P_{0}\right)$, and $\gamma(\mathcal{G} a l(M, v)=$ $\gamma_{2}(\mathcal{G} a l(K, v))=\mathcal{G} a l(P / M(t), v)$ for each $v \in S_{1}$. This completes the proof of the proposition.

\section{§11. Free product of local groups}

The group theoretic and field theoretic information gathered up to now gives a free product theorem: $\operatorname{Gal}(M)$ is a free product of local subgroups.

The homomorphism $\gamma: \operatorname{Gal}(M) \rightarrow \operatorname{Gal}(P / M(t))$ of Proposition 10.5 need not be surjective. We fix this drawback by assuming in the next result that $\operatorname{Gal}(P / M(t))=\left\langle\operatorname{Gal}\left(P / P_{0}\right), \operatorname{Gal}(P / M(t))\right\rangle_{v \in S_{1}}$.

For an extension $E$ of $K$ and a finite Galois extension $F$ of $E$ let $\mathcal{G a l}(F / E, S)=\bigcup_{v \in S} \mathcal{G a l}(F / E, v)$.

Proposition 11.1. Every finite locally solvable self-generated embedding problem of group piles

$$
(\varphi: \operatorname{Gal}(M, S) \rightarrow \mathbf{A}, \alpha: \mathbf{B} \rightarrow \mathbf{A}),
$$

is solvable.

Proof. Let $A_{0}=\varphi\left(\operatorname{Gal}\left(K_{0}\right)\right)$. Then $\mathcal{A}_{0}$ is the $A$-class generated by $A_{0}$ and $A=\left\langle A_{0}, \mathcal{A}_{1}\right\rangle$ (by Proposition 10.4(f)). By assumption, there exists $B_{0} \in \mathcal{B}_{0}$ such that $B=\left\langle B_{0}, \mathcal{B}_{1}\right\rangle$. In particular, $\alpha\left(B_{0}\right)$ is conjugate to $A_{0}$. Applying Lemma 5.2 , we may replace $B_{0}$ by a conjugate subgroup, if necessary, to assume that $\alpha\left(B_{0}\right)=A_{0}$. 
Now we may assume that $\mathbf{A}=(\operatorname{Gal}(N / M), \mathcal{G} a l(N / M, v))_{v \in S}$, where $N$ is a finite Galois extension of $M, \mathcal{G}$ al $(N / M, 0)=\left\{\operatorname{Gal}\left(N / N_{0}\right)^{\sigma} \mid \sigma \in\right.$ $\operatorname{Gal}(N / M)\}$ with $N_{0}=K_{0} \cap N$, and $\varphi$ is the restriction map $\operatorname{res}_{\tilde{M} / N}$.

Next we replace B by a group pile of Galois groups over $M(t)$ with $t$ transcendental over $M$. To this end we consider the deficient group piles

$$
\begin{aligned}
& \mathbf{H}=(\operatorname{Gal}(M(t)), \mathcal{G a l}(M(t), v))_{v \in S_{1}}, \\
& \mathbf{G}=(\operatorname{Gal}(M), \mathcal{G a l}(M, v))_{v \in S_{1}},
\end{aligned}
$$

where $\mathbf{G}$ is obtained from $\operatorname{Gal}(M, S)$ by replacing $\operatorname{Gal}(M, 0)$ by the class of the trivial group. Let $\lambda: \mathbf{H} \rightarrow \mathbf{G}$ be the restriction map. By Lemma 8.4(d), $\lambda$ is rigid. Let $\mathbf{A}^{\prime}=(\operatorname{Gal}(N / M), \mathcal{G a l}(N / M, v))_{v \in S_{1}}$ and $\mathbf{B}^{\prime}=\left(B, B_{v}\right)_{v \in S_{1}}$ be the deficient group piles associated with $\mathbf{A}$ and $\mathbf{B}$. Then

$$
\left(\varphi: \mathbf{G} \rightarrow \mathbf{A}^{\prime}, \alpha: \mathbf{B}^{\prime} \rightarrow \mathbf{A}^{\prime}\right)
$$

is a finite locally solvable embedding problem of deficient group piles. Proposition 10.4 implies that $\mathbf{G}$ is a separated deficient group pile satisfying Conditions (a)-(d) of Proposition 7.5. Proposition 10.3(f) settles Condition (e) of Proposition 7.5.

Thus, Proposition 7.5 gives an epimorphism of deficient group files $\delta: \mathbf{H} \rightarrow \mathbf{B}^{\prime}$ such that $\alpha \circ \delta=\varphi \circ \lambda$ and $\lambda(\operatorname{Ker}(\delta))=\operatorname{Ker}(\varphi)$. Let $P$ be the fixed field in $\widetilde{M(t)}$ of $\operatorname{Ker}(\delta)$. Then $P$ is a finite Galois extension of $M(t)$ regular over $N$ (Remark 7.6) and res $\widetilde{M(t)} / P$ maps $\mathbf{H}$ onto the deficient group pile $\mathbf{P}^{\prime}=(\operatorname{Gal}(P / M(t)), \mathcal{G a l}(P / M(t), v))_{v \in S_{1}}$. Moreover, there is an isomorphism $\bar{\delta}: \mathbf{P}^{\prime} \rightarrow \mathbf{B}^{\prime}$ of deficient group piles such that $\delta=\bar{\delta} \circ$ res $\widetilde{M(t)} / P$. Then $\alpha \circ \bar{\delta}=\operatorname{res}_{P / N}$. Let $P_{0}$ be the fixed field of $\bar{\delta}^{-1}\left(B_{0}\right)$ in $P$, and $\mathcal{G} a l(P / M(t), 0)=\left\{\operatorname{Gal}\left(P / P_{0}\right)^{\sigma} \mid \sigma \in \operatorname{Gal}(P / M(t))\right\}$. Then

$$
\mathbf{P}=(\operatorname{Gal}(P / M(t)), \mathcal{G a l}(M / P(t), v))_{v \in S}
$$

is a finite group pile and $\bar{\delta}: \mathbf{P} \rightarrow \mathbf{B}$ is an isomorphism of group piles such that $\alpha \circ \bar{\delta}=\operatorname{res}_{P / N}$. Replacing $\mathbf{B}$ by $\mathbf{P}$ via $\bar{\delta}$ we may assume that $B=$ $\operatorname{Gal}(P / M(t)), \mathcal{B}_{v}=\mathcal{G} a l(P / M(t), v)$ for each $v \in S$, and $\delta=\operatorname{res} \widetilde{M(t)} / P$. Then $\alpha$ is the restriction $\operatorname{res}_{P / N}, P_{0} \cap N=N_{0}$, and

$$
\operatorname{Gal}(P / M(t))=\left\langle\operatorname{Gal}\left(P / P_{0}\right), \mathcal{G} a l(P / M(t), v)\right\rangle_{v \in S_{1}}
$$

By Proposition 10.5, there is a homomorphism $\gamma: \operatorname{Gal}(M) \rightarrow \operatorname{Gal}(P /$ $M(t))$ of profinite groups such that $\alpha \circ \gamma=\varphi, \gamma\left(\operatorname{Gal}\left(K_{0}\right)\right)=\operatorname{Gal}\left(P / P_{0}\right)$, 
and $\gamma\left((\mathcal{G a l}(M, v))=\mathcal{G a l}(P / M(t), v)\right.$ for each $v \in S_{1}$. By $(3), \gamma$ is an epimorphism of group piles. Consequently, (1) has a solution.

Proposition 11.2. In the setup of 10.1 there exists for each $v \in S_{1} a$ closed subset $R_{v}$ of $\operatorname{Gal}(K)$ such that

$$
\operatorname{Gal}(M)=\operatorname{Gal}\left(K_{0}\right) * \underset{v \in S_{1}}{\prod_{\rho \in R_{v}}} \prod_{\operatorname{Gal}\left(K_{v}^{\rho}\right) .}
$$

Moreover, for each $v \in S_{1}, R_{v}$ is a system of representatives of $\operatorname{Gal}(K) /$ $\operatorname{Gal}(M)$ and $\left\{\operatorname{Gal}\left(K_{v}\right)^{\rho} \mid \rho \in R_{v}\right\}$ is a closed system of representatives for the $\operatorname{Gal}(M)$-orbits of $\mathcal{G} a l(M, v)$.

Proof. By Condition (1b) of Section 10, $\operatorname{Gal}\left(K_{0}\right)$ is a finitely generated free profinite group. By Lemma $8.4(\mathrm{c}), \operatorname{Gal}\left(K_{v}\right)$ is a finitely generated nontrivial group. Thus, the groups in $\mathcal{G a l}(K, v), v \in S$, satisfy the conditions of Data 5.1.

We prove that $\operatorname{Gal}(M, S)=(\operatorname{Gal}(M), \mathcal{G} a l(M, v)))_{v \in S}$ is a Cantor group pile over $\left(\operatorname{Gal}\left(K_{v}\right)\right)_{v \in S}$. In other words, we verify Condition (1) of Definition 6.1 for $\operatorname{Gal}(M, S)$.

Condition (1a) holds because $M$ is a countable field. Condition (1b) follows from Lemma 10.4(e). Lemma 10.4(c) implies Condition (1c), that is $\mathcal{G} a l(M, S)=\bigcup_{v \in S} \mathcal{G} a l(M, v)$, where $\mathcal{G} a l(M, v)$ is open-closed in $\operatorname{Gal}(M, S)$ and each group in $\mathcal{G} a l(M, v)$ is isomorphic to $\mathcal{G} a l\left(K_{v}\right)$. By Proposition $10.4(\mathrm{f}), \mathbf{G a l}(M, S)$ is self-generated. Finally, Proposition 11.1 completes the proof of $(1 \mathrm{~d})$.

It follows from Proposition 6.4 that $\operatorname{Gal}(M)=\operatorname{Gal}\left(K_{0}\right) *{ }_{v \in S_{1}} \circledast_{t \in T_{v}} G_{t}$, where $T_{v}$ is a Cantor space and $\mathcal{T}_{v}=\left\{G_{t} \mid t \in T_{v}\right\}$ is a closed system of representatives of the $\operatorname{Gal}(M)$-classes of $\mathcal{G} a l(M, v)$.

For each $v \in S_{1}$ let $s: \operatorname{Gal}(K) \rightarrow \mathcal{G} a l(M, v)$ be the map defined by $s(\rho)=K_{v}^{\rho}$. It is continuous and surjective. Moreover, $\operatorname{Gal}(M)$ acts on $\operatorname{Gal}(K)$ by multiplication from the right and on $\mathcal{G} a l(M, v)$ by upper right conjugation and $s$ respects this action. Since $K$ is countable, $\operatorname{Gal}(K)$ has a countable basis for its topology. Hence, $s$ has a continuous section $s^{\prime}: \mathcal{G a l}(M, v) \rightarrow \operatorname{Gal}(K)$ [Har, Lemma 8.1]. Thus, $R_{v}=s^{\prime}\left(\mathcal{T}_{v}\right)$ is a closed system of representatives of $\operatorname{Gal}(K) / \operatorname{Gal}(M)$ and $\left\{\operatorname{Gal}\left(K_{v}^{\rho}\right) \mid \rho \in R_{v}\right\}=\mathcal{T}_{v}$ is a closed system of representatives of the $\operatorname{Gal}(M)$-orbits of $\mathcal{G} a l(M, v)$. Note that the map $t \mapsto G_{t}$ of $T_{v}$ onto $\mathcal{T}_{v}$ is a continuous bijection of profinite spaces, hence it is an homeomorphism. It follows that the map $t \rightarrow s^{\prime}\left(G_{t}\right)$ 
of $T_{v}$ is a homeomorphism satisfying $G_{t}=\operatorname{Gal}\left(K_{v}^{s^{\prime}\left(G_{t}\right)}\right)$. Consequently, $\operatorname{Gal}(M)=\operatorname{Gal}\left(K_{0}\right) * \mathbb{1}_{v \in S_{1}} \mathbb{1}_{\rho \in R_{v}} \operatorname{Gal}\left(K_{v}^{\rho}\right)$.

\section{§12. Large fields chosen at random}

As in Setup 10.1 let $K$ be a countable Hilbertian field of characteristic 0 , and $S=\{0\} \cup S_{1}$, where $S_{1}$ is a finite set independent classical P-adic valuations and orderings. In addition, let $e \geq 0$ an integer. We prove below that for almost all $\boldsymbol{\sigma} \in \operatorname{Gal}(K)^{e}$, the field $K_{0}=\tilde{K}(\boldsymbol{\sigma})$ satisfies Condition (1) of Section 10. Applying Proposition 11.2, we get a presentation of $\operatorname{Gal}\left(K_{\text {tot, } S_{1}}(\boldsymbol{\sigma})\right)$ as a free product of local groups.

LEMma 12.1. Let $K$ be a countable Hilbertian field, $S_{1}$ a finite set of independent orderings and valuations, and $e \geq 0$ an integer. Then for almost all $\boldsymbol{\sigma} \in \operatorname{Gal}(K)^{e}$ the field $K_{s}(\boldsymbol{\sigma})$ is $P A C$ over each set $H \cap A$, where $H$ is a Hilbert subset of $K^{r}$ and $A$ is a nonempty open $S_{1}$-adic subset of $K^{r}$.

Proof. Let $L$ be a finite separable extension of $K, f \in L\left[T_{1}, \ldots, T_{r}, X\right]$ an absolutely irreducible polynomial, $g \in L\left[T_{1}, \ldots, T_{r}\right]$ a nonzero polynomial, $A$ a nonempty $S_{1}$-open subset of $K^{r}$, and $H$ a separable Hilbert subset of $K^{r}$. Set

$$
\begin{array}{r}
C(L, f, g, A, H)=\left\{\boldsymbol{\sigma} \in \operatorname{Gal}(L)^{e} \mid\right. \\
\text { there exist } \mathbf{a} \in H \cap A \text { and } b \in K_{s}(\boldsymbol{\sigma}) \\
\text { such that } f(\mathbf{a}, b)=0 \text { and } g(\mathbf{a}) \neq 0\}
\end{array}
$$

and let $\mu_{L}$ be the normalized Haar measure of $\operatorname{Gal}(L)^{e}$.

Claim: $\mu_{L}(C(L, f, g, A, H))=1$. To prove the claim, we construct by induction a linearly disjoint sequence of separable extensions $L_{1}, L_{2}, L_{3}, \ldots$ of $L$ such that for each $i,\left[L_{i}: L\right]=\operatorname{deg}_{X}(f)$ and there exist a $\in H \cap A$ and $b \in K_{s}(\boldsymbol{\sigma})$ with $f(\mathbf{a}, b)=0$ and $g(\mathbf{a}) \neq 0$. Suppose we have already constructed $L_{1}, \ldots, L_{n}$ with that property and let $L^{\prime}=L_{1} \cdots L_{n}$. Since $f$ is absolutely irreducible, the set $H^{\prime}$ of all $\mathbf{a} \in\left(L^{\prime}\right)^{r}$ such that $f(\mathbf{a}, X)$ is irreducible and separable over $L^{\prime}$ and $g\left(\mathbf{a}^{\prime}\right) \neq 0$ is a separable Hilbert subset of $\left(L^{\prime}\right)^{r}$. By [FrJ, Cor. 12.2.3], $H \cap H^{\prime}$ contains a separable Hilbert subset $H_{K}$ of $K^{r}$. By [Jar2, Prop. 19.8], we may choose a $\in H_{K} \cap A$ such that $g(\mathbf{a}) \neq 0$. Let $b \in K_{s}$ with $f(\mathbf{a}, b)=0$ and set $L_{n+1}=K(b)$. Then $L_{n+1}$ is linearly disjoint from $L^{\prime}$ over $K$, hence $L_{1}, \ldots, L_{n+1}$ are disjoint over $K$. This completes the induction. 
By [FrJ, Lemma 18.5.3], for almost all $\boldsymbol{\sigma} \in \operatorname{Gal}(L)^{e}$ the field $L_{s}(\boldsymbol{\sigma})$ contains at least one of the fields $L_{i}$. Hence $\mu_{L}(C(L, f, g, A, H))=1$, as asserted.

Since $K$ is countable, there are only countably many $L, f, g, H$ as above. For each valuation or ordering $v$ of $K$ the set of $v$-open discs is countable and forms a basis for the $v$-topology of $K$. Hence, the set of all rectangles of $v$-open discs with $v \in S_{1}$ is countable and forms a basis $\mathcal{A}_{L}$ for the $S_{1}$-topology of $K^{r}$. It follows that the set

$$
C=\operatorname{Gal}(K)^{e} \backslash \bigcup_{L, f, g, A, H}\left(\operatorname{Gal}(L)^{e} \backslash C(L, f, g, A, H)\right)
$$

where $L, f, g, H$ are as above and $A$ ranges over $\mathcal{A}_{L}$ has measure 1 in $\operatorname{Gal}(K)^{e}$. Each $\boldsymbol{\sigma} \in C$ has the desired property.

Given a field $K$ and $\sigma_{1}, \ldots, \sigma_{e} \in \operatorname{Gal}(K)$, we denote the maximal Galois extension of $K$ in $K_{s}(\boldsymbol{\sigma})$ by $K_{s}[\boldsymbol{\sigma}]$.

Lemma 12.2. Let $K$ be a countable Hilbertian field, $S_{1}$ a finite set of independent classical $\mathrm{P}$-adic valuations and orderings, and $e \geq 0$. Then, for almost all $\boldsymbol{\sigma} \in \mathrm{Gal}(K)^{e}$ each field $M$ lying between $K_{\mathrm{tot}, S_{1}}[\boldsymbol{\sigma}]$ and $K_{\mathrm{tot}, S_{1}}$ is $P S_{1} C$.

Proof. By [GeJ, Theorem A], for almost all $\boldsymbol{\sigma} \in \mathrm{Gal}(K)^{e}$ the field $L=K_{\text {tot }, S_{1}}[\boldsymbol{\sigma}]$ is $\mathrm{P} S_{1} \mathrm{C}$. In the notation of [Jar1, Section 7], this means that $L$ is $\mathrm{PKC}$, where $\mathcal{K}=\operatorname{Alg} \operatorname{Ext}\left(K, S_{1}\right)$. By Lemma 10.3(d), $\operatorname{Alg} \operatorname{Ext}\left(K, S_{1}\right)$ is closed in $\operatorname{Alg} \operatorname{Ext}(K)$. If a field $M$ lies between $K_{s}[\boldsymbol{\sigma}]$ and $K_{\text {tot, } S_{1}}$, then $M \bar{K}=\bar{K}$ for each $\bar{K} \in \operatorname{AlgExt}\left(L, S_{1}\right)$. Hence, by [Jar1, Lemma 7.4], $M$ is $\mathrm{P} S_{1} \mathrm{C}$.

Proposition 12.3. Let $K$ be a countable Hilbertian field of characteristic $0, e \geq 0$ an integer, and $S_{1}$ a finite set of independent classical $\mathrm{P}$-adic valuations and orderings. Then for almost all $\boldsymbol{\sigma} \in \operatorname{Gal}(K)^{e}$ the fields $K_{0}=\tilde{K}(\boldsymbol{\sigma})$ and $M=K_{\mathrm{tot}, S_{1}}(\boldsymbol{\sigma})$ satisfy Condition (1) of Section 10.

Proof of (1a) of Section 10. This is a special case of Lemma 12.1.

Proof of (1b) of Section 10. By [FrJ, Thm. 18.5.6], $\operatorname{Gal}(\tilde{K}(\boldsymbol{\sigma})) \cong \hat{F}_{e}$ for almost all $\boldsymbol{\sigma} \in \operatorname{Gal}(K)^{e}$.

Proof of (1c) of Section 10. This is a special case of Lemma 12.2. 
Proof of (1d) of Section 10. Let $n$ be a positive integer and consider the general polynomial $f(\mathbf{T}, X)=X^{n}+T_{1} X^{n-1}+\cdots+T_{n}$ of degree $n$. Its Galois group over $K(\mathbf{T})$ is isomorphic to $S_{n}$ [Lan, p. 272, Example 4]. By [FrJ, Lemma 13.1.1], there exists a separable Hilbert subset $H$ of $K^{n}$ such that for each $\mathbf{a} \in H$, the polynomial $f(\mathbf{a}, X)$ is Galois over $K$ with Galois group isomorphic to $S_{n}$. Consider also the polynomial $g(X)=\prod_{i=1}^{n}(X-i)=$ $X^{n}+c_{1} X^{n-1}+\cdots+c_{n}$ with $c_{1}, \ldots, c_{n} \in \mathbb{Z}$. The theorem about the continuity of roots [Jar2, Prop. 12.3] gives an $S_{1}$-adic open neighborhood $A$ of $\mathbf{c} \in K^{n}$ such that for each $v \in S_{1}$ and each $\mathbf{a} \in A$ the polynomial $f(\mathbf{a}, X)$ totally splits in $K_{v}$. It follows that $f(\mathbf{a}, X)$ totally splits in $K_{\text {tot, } S_{1}}$.

By (1a) of Section 10 (which we have already proved), for almost all $\boldsymbol{\sigma} \in \operatorname{Gal}(K)^{e}$ there exist $\mathbf{a} \in H \cap A$ and $b \in K_{0}=\tilde{K}(\boldsymbol{\sigma})$ such that $f(\mathbf{a}, b)=0$. By the choice of $A$ and $H$, the field $K(b)$ is Galois over $K$ with Galois group $S_{n}$ and $K(b) \subseteq K_{\text {tot }, S_{1}}$, so $K(b) \subseteq M=K_{\text {tot }, S_{1}}(\boldsymbol{\sigma})$.

The compositum of all $K(b)$ with $n$ ranges over all positive integers is an infinite Galois extension of $K$ in $M$.

Proposition 12.3 allows us now to apply Proposition 11.2 to $\tilde{K}(\boldsymbol{\sigma})$ for almost all $\boldsymbol{\sigma} \in \operatorname{Gal}(K)^{e}$ and to achieve the main result of this work.

THEOREM 12.4. Let $K$ be a countable Hilbertian field of characteristic $0, e \geq 0$ an integer, and $S_{1}$ a finite set of independent classical P-adic valuations and orderings of $K$. Then, for almost all $\boldsymbol{\sigma} \in \operatorname{Gal}(K)^{e}$ there exists for each $v \in S_{1}$ a closed subset $R_{v}$ of $\mathrm{Gal}(K)$ such that

$$
\operatorname{Gal}\left(K_{\text {tot }, S_{1}}(\boldsymbol{\sigma})\right)=\operatorname{Gal}(\tilde{K}(\boldsymbol{\sigma})) * \prod_{v \in S_{1}} \prod_{\rho \in R_{v}} \operatorname{Gal}\left(K_{v}^{\rho}\right) .
$$

Moreover, $R_{v}$ is a system of representatives of $\mathrm{Gal}(K) / \operatorname{Gal}\left(K_{\mathrm{tot}, S_{1}}(\boldsymbol{\sigma})\right)$ and $\left\{K_{v}^{\rho} \mid \rho \in R_{v}\right\}$ is a closed system of representatives for the $\operatorname{Gal}\left(K_{\mathrm{tot}, S_{1}}(\boldsymbol{\sigma})\right)$ orbits of $\operatorname{Alg} \operatorname{Ext}(K, v)$.

Remark 12.5. The fields $K_{\text {tot, } S_{1}}[\boldsymbol{\sigma}]$. Given $K, S_{1}$, and $e \geq 1$ as in Theorem 12.4, we would like to prove the following analog of Theorem 12.4:

For almost all $\boldsymbol{\sigma} \in \operatorname{Gal}(K)^{e}$ and for each $v \in S_{1}$ there exists a closed subsets $R_{v}$ of $\operatorname{Gal}(K)$ such that

$$
\operatorname{Gal}\left(K_{\text {tot }, S_{1}}[\boldsymbol{\sigma}]\right)=\operatorname{Gal}(\tilde{K}[\boldsymbol{\sigma}]) * \prod_{v \in S_{1}} \prod_{\rho \in R_{v}} \operatorname{Gal}\left(K_{v}^{\rho}\right) .
$$


We know that for almost all $\boldsymbol{\sigma} \in \operatorname{Gal}(K)^{e}$ the field $\tilde{K}[\boldsymbol{\sigma}]$ is PAC and $\operatorname{Gal}(\tilde{K}[\boldsymbol{\sigma}]) \cong \hat{F}_{\omega}$ [Jar3, Lemma 2.7]. However, since $\tilde{K}[\boldsymbol{\sigma}]$ is Galois over $K$, and not algebraically closed, it is not PAC over $K$, at least if $K$ is finitely generated over $\mathbb{Q}$ (the case where $K$ is a number field is proved in [Jar4, Main Theorem], the general case is [BSJ, Thm. B]). In particular, $\tilde{K}[\boldsymbol{\sigma}]$ does not satisfy Condition (1a) of Section 10, so a major argument in Part D of the proof of Lemma 9.5 does not work in the new case. Consequently, the proof of Theorem 12.4 cannot be adapted to a proof of (2) and one has to come up with another strategy.

\section{REFERENCES}

[BSJ] L. Bary-Soroker and M. Jarden, PAC fields over finitely generated fields, Mathematische Zeitschrift, 260 (2008), 329-334.

[Efr] I. Efrat, Lifting of Generating Subgroups, Proceedings of the American Mathematical Society, 125 (1997), 2217-2219.

[FHV] M. Fried, D. Haran, and H. Völklein, Absolute Galois group of the totally real numbers, C. R. Acad. Sci. Paris, 317 (1993), 995-999.

[FrJ] M. D. Fried and M. Jarden, Field Arithmetic, Third Edition, revised by Moshe Jarden, Ergebnisse der Mathematik (3) 11, Springer, Heidelberg, 2008.

[Gey] W.-D. Geyer, Galois groups of intersections of local fields, Israel Journal of Mathematics, 30 (1978), 382-396.

[GeJ] W.-D. Geyer and M. Jarden, PSC Galois extensions of Hilbertian fields, Mathematische Nachrichten, 236 (2002), 119-160.

[GPR] B. Green, F. Pop, and P. Roquette, On Rumely's local-global principle, Jahresbericht der Deutschen Mathematiker-Vereinigung, 97 (1995), 43-74.

[Har] D. Haran, On closed subgroups of free products of profinite groups, Proceedings of the London Mathematical Society, 55 (1987), 266-289.

[HaJ1] D. Haran and M. Jarden, The absolute Galois group of a pseudo real closed algebraic field, Pacific Journal of Mathematics, 123 (1986), 55-69.

[HaJ2] D. Haran and M. Jarden, The absolute Galois group of a pseudo p-adically closed field, Journal für die reine und angewandte Mathematik, 383 (1988), 147-206.

[HaJ3] D. Haran and M. Jarden, Regular split embedding problems over complete valued fields, Forum Mathematicum, 10 (1998), 329-351.

[HJPa] D. Haran, M. Jarden, and F. Pop, Projective group structures as absolute Galois structures with block approximation, Memoirs of AMS, 189 (2007), 1-56.

[HJPb] D. Haran, M. Jarden, and F. Pop, P-adically projective groups as absolute Galois groups, International Mathematics Research Notices, 32 (2005), 1957-1995.

[Jar1] M. Jarden, Algebraic realization of p-adically projective groups, Compositio Mathematica, 79 (1991), 21-62.

[Jar2] M. Jarden, Intersection of local algebraic extensions of a Hilbertian field (A. Barlotti et al., eds.), NATO ASI Series C, 333, Kluwer, Dordrecht, 1991, pp. 343-405. 
[Jar3] M. Jarden, Large normal extensions of Hilbertian fields, Mathematische Zeitschrift, 224 (1997), 555-565.

[Jar4] M. Jarden, PAC fields over number fields, Journal de Théorie des Nombres de Bordeaux, 18 (2006), 371-377.

[JaR1] M. Jarden and A. Razon, Pseudo algebraically closed fields over rings, Israel Journal of Mathematics, 86 (1994), 25-59.

[JaR2] M. Jarden and A. Razon, Rumely's local global principle for algebraic PSC fields over rings, Transactions of AMS, 350 (1998), 55-85.

[Lan] S. Lang, Algebra, Third Edition, Eddison-Wesley, Reading, 1993.

[Mel] O. V. Melnikov, Subgroups and homology of free products of profinite groups, Math. USSR Izvestiya, 34 (1990), 97-119.

[Pop1] F. Pop, Galoissche Kennzeichnung p-adisch abgeschlossener Körper, Journal für die reine und angewandte Mathematik, 392 (1988), 145-175.

[Pop2] F. Pop, Fields of totally $\Sigma$-adic numbers, manuscript, Heidelberg, 1992

[Pop3] F. Pop, On prosolvable subgroups of profinite free products and some applications, manuscripta mathematica, 86 (1995), 125-135.

[Pop4] F. Pop, Embedding problems over large fields, Annals of Mathematics, 144 (1996), $1-34$.

[Pre] A. Prestel, Lectures on Formally Real Fields, Lecture Notes in Mathematics 1093, Springer, Berlin, 1984.

[PrR] A. Prestel and P. Roquette, Formally $p$-adic Fields, Lecture Notes in Mathematics 1050, Springer, Berlin, 1984.

Dan Haran

School of Mathematics

Tel Aviv University

Ramat Aviv, Tel Aviv 69978

Israel

haran@post.tau.ac.il

Moshe Jarden

School of Mathematics

Tel Aviv University

Ramat Aviv, Tel Aviv 69978

Israel

jarden@post.tau.ac.il

Florian Pop

Department of Mathematics

University of Pennsylvania

Philadelphia, PA 19104-6395

USA

pop@math.upenn.edu 